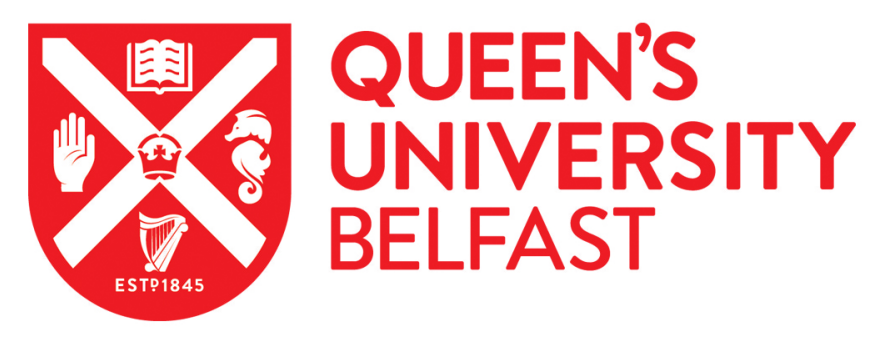

\title{
Positron-molecule interactions: resonant attachment, annihilation, and bound states
}

Gribakin, G. F., Young, J. A., \& Surko, C. M. (2010). Positron-molecule interactions: resonant attachment, annihilation, and bound states. Reviews of Modern Physics, 82(3), 2557-2607.

https://doi.org/10.1103/RevModPhys.82.2557

\section{Published in:}

Reviews of Modern Physics

Document Version:

Publisher's PDF, also known as Version of record

Queen's University Belfast - Research Portal:

Link to publication record in Queen's University Belfast Research Portal

\section{Publisher rights}

Copyright 2010 American Physical Society.

This work is made available online in accordance with the publisher's policies. Please refer to any applicable terms of use of the publisher.

\section{General rights}

Copyright for the publications made accessible via the Queen's University Belfast Research Portal is retained by the author(s) and / or other copyright owners and it is a condition of accessing these publications that users recognise and abide by the legal requirements associated with these rights.

Take down policy

The Research Portal is Queen's institutional repository that provides access to Queen's research output. Every effort has been made to ensure that content in the Research Portal does not infringe any person's rights, or applicable UK laws. If you discover content in the Research Portal that you believe breaches copyright or violates any law, please contact openaccess@qub.ac.uk. 


\title{
Positron-molecule interactions: Resonant attachment, annihilation, and bound states
}

\author{
G. F. Gribakin* \\ Department of Applied Mathematics and Theoretical Physics, Queen's University Belfast, \\ Belfast, BT7 1NN, Northern Ireland, United Kingdom
}

\author{
J. A. Young ${ }^{\dagger}$ \\ Jet Propulsion Laboratory, California Institute of Technology, 4800 Oak Grove Drive, \\ Pasadena, California 91109, USA \\ C. M. Surko ${ }^{\ddagger}$ \\ Department of Physics, University of California, San Diego, 9500 Gilman Drive, La Jolla, \\ California 92093-0319, USA
}

(Published 14 September 2010)

\begin{abstract}
This article presents an overview of current understanding of the interaction of low-energy positrons with molecules with emphasis on resonances, positron attachment, and annihilation. Measurements of annihilation rates resolved as a function of positron energy reveal the presence of vibrational Feshbach resonances (VFRs) for many polyatomic molecules. These resonances lead to strong enhancement of the annihilation rates. They also provide evidence that positrons bind to many molecular species. A quantitative theory of VFR-mediated attachment to small molecules is presented. It is tested successfully for selected molecules (e.g., methyl halides and methanol) where all modes couple to the positron continuum. Combination and overtone resonances are observed and their role is elucidated. Molecules that do not bind positrons and hence do not exhibit such resonances are discussed. In larger molecules, annihilation rates from VFR far exceed those explicable on the basis of single-mode resonances. These enhancements increase rapidly with the number of vibrational degrees of freedom, approximately as the fourth power of the number of atoms in the molecule. While the details are as yet unclear, intramolecular vibrational energy redistribution (IVR) to states that do not couple directly to the positron continuum appears to be responsible for these enhanced annihilation rates. In connection with IVR, experimental evidence indicates that inelastic positron escape channels are relatively rare. Downshifts of the VFR from the vibrational mode energies, obtained by measuring annihilate rates as a function of incident positron energy, have provided binding energies for 30 species. Their dependence upon molecular parameters and their relationship to positron-atom and positron-molecule binding-energy calculations are discussed. Feshbach resonances and positron binding to molecules are compared with the analogous electron-molecule (negative-ion) cases. The relationship of VFR-mediated annihilation to other phenomena such as Doppler broadening of the gamma-ray annihilation spectra, annihilation of thermalized positrons in gases, and annihilation-induced fragmentation of molecules is discussed. Possible areas for future theoretical and experimental investigation are also discussed.
\end{abstract}

DOI: $10.1103 /$ RevModPhys.82.2557 PACS number(s): 34.80.Lx, 34.50. $-\mathrm{s}, 71.60 .+\mathrm{z}, 78.70 . \mathrm{Bj}$

\section{CONTENTS}

I. Introduction and Overview

2558

II. Theory

2561

A. Annihilation basics

2561

B. Gamma-ray spectra and annihilation rates

2562

C. Positron-molecule wave function

2563

D. Direct annihilation: Virtual and weakly bound positron states

2564

E. Resonant annihilation

2566

F. Resonances due to infrared-active modes
G. Resonant annihilation in large molecules

1. Vibrational level densities

2. Mode-based resonant doorway states

3. Annihilation and the onset of IVR

H. Calculations of annihilation and binding

1. Annihilation

2. Positron-molecule binding

III. Experimental Tools and Procedures
A. Annihilation-rate measurements with thermalized positrons in atmospheric pressure gases
B. Buffer-gas positron traps as tailored sources of positrons
C. Annihilation-rate measurements in positron traps
D. Trap-based cold positron beams
E. Energy-resolved annihilation measurements
F. Gamma-ray spectral measurements
G. Annihilation-induced fragmentation 


\begin{tabular}{|c|c|}
\hline IV. Annihilation on Small Molecules & 2579 \\
\hline A. Halomethanes as a benchmark example of VFR & 2580 \\
\hline B. Methanol: A case of multimode VFR & 2581 \\
\hline C. VFR from dipole-forbidden vibrations & 2582 \\
\hline D. Effect of molecular size on the magnitudes of VFR & 2583 \\
\hline E. Nonresonant annihilation in small molecules & 2583 \\
\hline F. Small molecule summary & 2585 \\
\hline \multicolumn{2}{|l|}{ V. IVR-Enhanced Resonant Annihilation in Larger } \\
\hline Molecules & 2585 \\
\hline A. Overview & 2585 \\
\hline B. The alkane molecule paradigm & 2586 \\
\hline C. Dependence of $Z_{\text {eff }}$ on molecular size & 2587 \\
\hline D. Toward a model of annihilation in large molecules & 2589 \\
\hline E. Inelastic autodetachment & 2589 \\
\hline 1. Fluorine-substituted alkanes & 2589 \\
\hline 2. Effects of molecular temperature on $Z_{\text {eff }}$ & 2590 \\
\hline F. Other IVR-related phenomena & 2591 \\
\hline G. Large molecule summary & 2592 \\
\hline VI. Positron-Molecule Binding Energies & 2592 \\
\hline A. Relation to molecular properties & 2592 \\
\hline $\begin{array}{l}\text { B. Comparison with negative ions of molecules and } \\
\text { clusters }\end{array}$ & 2595 \\
\hline \multicolumn{2}{|l|}{ VII. Analysis of Annihilation Rates Measured with } \\
\hline Thermalized Positrons & 2596 \\
\hline VIII. Other Topics & 2597 \\
\hline A. Gamma-ray Doppler-broadening measurements & 2597 \\
\hline B. Annihilation-induced fragmentation of molecules & 2600 \\
\hline $\begin{array}{l}\text { C. Nonlinear dependence of annihilation on molecular } \\
\text { density }\end{array}$ & 2601 \\
\hline IX. Summary and a Look to the Future & 2602 \\
\hline owledgments & 2603 \\
\hline & 2604 \\
\hline
\end{tabular}

\section{INTRODUCTION AND OVERVIEW}

The subject of this review is the interaction of lowenergy positrons with molecules. Positrons, the antiparticles of electrons, are important in many areas of science and technology. Much of their utility relies on the fact that, when an electron and positron interact, they can annihilate, producing a characteristic burst of gamma rays. The lowest order process results in two back-to-back photons, each with the energy of the rest mass of the electron (or positron) $(511 \mathrm{keV})$.

The annihilation of low-energy (e.g., $\leqslant 50 \mathrm{eV}$ ) positrons on atoms and molecules plays a particularly important role in many fields. In medicine, positron emission tomography (PET) exploits two-gamma annihilation to study human metabolic processes (Wahl, 2002). In material science, there are numerous positron-based techniques to study the properties of matter (Schultz and Lynn, 1988; Puska and Nieminen, 1994; Dupasquier and Mills, 1995; Coleman, 2000), including the Fermi surfaces in metals (Major et al., 2004), microscopic pores in solids (Gidley et al., 2000, 2006), the free volume in polymers (Dlubek et al., 1998), and the composition and structure of surfaces (David et al., 2001). In astronomy, $511 \mathrm{keV}$ annihilation radiation (the strongest gammaray line of extraterrestrial origin) has been proven to be useful in elucidating astrophysical processes (Ramaty et al., 1992; Churazov et al., 2005; Guessoum et al., 2010). A current research goal is the creation of a Bose condensate of positronium (Ps) atoms (i.e., the electronpositron analog of the hydrogen atom) that offers promise for the development of an annihilation gamma-ray laser (Mills, 2002, 2007; Mills et al., 2004; Cassidy and Mills, 2007).

Typically, positrons from conventional sources (e.g., radioisotopes or electron accelerators) slow down from energies of kilovolts to hundreds of kilovolts to $\$ 50 \mathrm{eV}$ before annihilating. In the case of atoms or molecules, if the incident positron energy $\varepsilon$ is greater than the Psformation threshold $E_{\mathrm{th}}=E_{i}-E_{\mathrm{Ps}}$, where $E_{i}$ is the ionization energy of the target and $E_{\mathrm{Ps}}=6.8 \mathrm{eV}$ is the binding energy of the ground-state Ps atom, then the dominant annihilation process is through Ps formation. The resulting Ps atom subsequently annihilates by emitting two or three gamma-ray quanta.

In this review, attention is restricted to positron energies below the Ps-formation threshold, $0<\varepsilon<E_{\text {th }}$, where the Ps channel is closed. Here annihilation occurs as a result of the overlap of the positron and electron densities during the collision. The basic rate in this case is the Dirac rate $\lambda_{D}$ for two-gamma annihilation in a freeelectron gas (Dirac, 1930)

$$
\lambda_{D}=\pi r_{0}^{2} c n_{e}
$$

where $r_{0}$ is the classical electron radius, $r_{0}=e^{2} / m c^{2}$ in cgs units, $e$ and $m$ are the electron charge and mass, $c$ is the speed of light, and $n_{e}$ is the electron density.

In his seminal discovery of the positronium atom, Deutsch (1951a, 1951b) found a curious effect. Although the annihilation rate for thermal positrons at $300 \mathrm{~K}$ in atomic and molecular gases was approximately in accord with Eq. (1) for some species (e.g., argon and nitrogen), the rate for dichlorofluoromethane $\mathrm{CCl}_{2} \mathrm{~F}_{2}$ ("freon-12") was much larger. Deutsch insightfully ascribed this effect to some type of resonant positron-molecule attachment process. A decade later, Paul and Saint-Pierre (1963) measured annihilation rates in gases of alkane molecules $\mathrm{C}_{n} \mathrm{H}_{2 n+2}$, from methane to butane, $n=1-4$. They found that the rate $\lambda$ was much greater than $\lambda_{D}$ and that the ratio $\lambda / \lambda_{D}$ increased exponentially with molecular size.

Annihilation rates in gases are conventionally normalized to the Dirac rate. The corresponding dimensionless quantity is the "effective number of electrons"

$$
Z_{\text {eff }}=\frac{\lambda}{\pi r_{0}^{2} c n},
$$

where $n$ is the density of atoms or molecules (Pomeranchuk, 1949; Fraser, 1968). For a simple collision and neglecting electron-positron correlations, one might expect that $\lambda \sim \lambda_{D}$ so that $Z_{\text {eff }}$ is comparable to $Z=n_{e} / n$, the

\footnotetext{
${ }^{1}$ In chemical kinetics, $Z_{\text {eff }}$ corresponds to the (normalized) rate constant of the annihilation reaction. In positron physics this quantity is commonly referred to as the "annihilation rate."
} 
total number of electrons per target atom or molecule. However, values of $Z_{\text {eff }}$ are often much larger (e.g., for butane, $Z_{\text {eff }} / Z=600$ ).

Positron annihilation in atoms and molecules was subsequently studied for a wide range of species (Osmon, 1965a, 1965b; Tao, 1965, 1970; McNutt et al., 1975; Sharma and McNutt, 1978; Charlton et al., 1980, 2002, 2006; Heyland et al., 1985, 1986; Sharma et al., 1985; AlQaradawi et al., 2000). Early experiments were done with thermal positrons in gases at atmospheric densities, $n \sim 1$ amagat (Deutsch, 1953; Paul and Saint-Pierre, 1963; Griffith and Heyland, 1978). ${ }^{2}$ Later, experiments were done at much lower densities using positrons trapped and cooled to $300 \mathrm{~K}$ (Surko, Passner, et al., 1988; Murphy and Surko, 1991; Iwata et al., 1995; Iwata, Greaves, and Surko, 1997). The experiments showed that the annihilation rates for many molecular species exceeded greatly the naive benchmark rate, $Z_{\text {eff }} \sim Z$, and a number of chemical trends were identified.

Since Deutsch's first results, these large annihilation rates were associated with some kind of resonance phenomenon or attachment process. Goldanskii and Sayasov (1964) discussed the possibility of resonanceenhanced annihilation due to a bound or virtual positron state close to zero energy. Smith and Paul (1970) considered the possibility that the large annihilation rates in molecules were due to a vibrational resonance, and several other explanations were proposed (Surko, Passner, et al., 1988; Dzuba et al., 1996; Laricchia and Wilkin, 1997; Gribakin, 2000). However, progress was hampered greatly by the lack of data other than for positrons with thermal energy distributions at $300 \mathrm{~K}$. The summary statement in 1982 by Sir Harrie Massey was that annihilation studies were "completely mysterious at present in almost all substances" (Fraser et al., 1982; Massey, 1982), and this remained more or less correct for another 20 years.

In the broader view, processes that are commonplace in physics involving matter, such as low-energy two-body scattering events, have frequently been found to be frustratingly difficult to study when antiparticles are involved (Schultz and Lynn, 1988; Eades and Hartmann, 1999; Coleman, 2000; Charlton and Humberston, 2001). The advent of efficient positron traps marked a turning point (Surko, Leventhal, et al., 1988; Murphy and Surko, 1992; Surko et al., 2005), enabling a new generation of studies (Surko, Passner, et al., 1988; Murphy and Surko, 1991; Iwata et al., 1995; Kurz et al., 1996; Iwata, Greaves, and Surko, 1997). Experiments with trapped positrons cooled to $300 \mathrm{~K}$ permitted studies of test species at low densities (e.g., $\leqslant 10^{-6}$ amagat). This ensured that annihilation was strictly due to binary collisions rather than many-particle effects (Iakubov and Khrapak, 1982), and it enabled study of a broader range of chemical species, including low-vapor-pressure liquids and solids.

\footnotetext{
${ }^{2} 1$ amagat $=2.69 \times 10^{19} \mathrm{~cm}^{-3}$ is the density of an ideal gas at standard temperature and pressure, $273.15 \mathrm{~K}$ and $101.3 \mathrm{kPa}$, respectively.
}

Gamma-ray spectra were measured for many molecules (Iwata, Greaves, and Surko, 1997). The $Z_{\text {eff }} / Z$ ratios were found to increase rapidly with molecular size up to species as large as naphthalene and hexadecane $\left(\mathrm{C}_{16} \mathrm{H}_{34}\right)$, reaching values $\gtrsim 10^{4}$ (Surko, Passner, et al., 1988; Murphy and Surko, 1991). ${ }^{3}$

A key to further progress was the development of a trap-based positron beam with a narrow energy spread ( $40 \mathrm{meV}$ ) (Gilbert et al., 1997; Kurz et al., 1998). Using this beam, annihilation rates for atoms and molecules were measured as a function of incident positron energy from $50 \mathrm{meV}$ to many electron volts. The result was the discovery of resonances associated with the molecular vibrational modes, namely, vibrational Feshbach resonances (VFRs) (Gilbert et al., 2002).

A crucial point is that VFRs generally require the existence of a bound state of the positron and the molecule. They occur when the incident positron excites a vibrational mode and simultaneously makes a transition from the continuum into the bound state. The existence of both low-lying vibrational excitations and a positron bound state thus enables the formation of long-lived positron-molecule resonant complexes in a two-body collision. The lifetime of these quasibound states is limited by positron autodetachment accompanied by vibrational deexcitation. The upper limit on the lifetime is $\leq 0.1 \mathrm{~ns}$, set by the positron annihilation rate in the presence of atomic-density electrons.

The annihilation rate as a function of positron energy $Z_{\text {eff }}(\varepsilon)$ (i.e., the "annihilation spectrum") for the fourcarbon alkane, butane, is shown in Fig. 1 (Gilbert et al., 2002). While there is some qualitative correspondence between the $Z_{\text {eff }}(\varepsilon)$ and the infrared (IR) absorption spectrum of the molecule, the shapes of the spectral features are quite different (Barnes et al., 2003).

These positron VFRs can be compared to resonances that play an important role in electron attachment to molecules and clusters (Christophorou et al., 1984; Hotop et al., 2003). The electron collision results in the production of long-lived (metastable) parent anions or molecular fragment negative ions via dissociative attachment. A dominant mechanism of electron capture by molecules is via negative-ion resonant states (Christophorou et al., 1984). Dissociative attachment usually proceeds via electron shape resonances of ground or electronically excited molecules. Such resonances are quite common in diatomic, triatomic, and polyatomic species at energies in the range $\sim 0-4 \mathrm{eV}$. The theoretical description of them involves (complex) BornOppenheimer potential-energy surfaces (O'Malley, 1966; Bardsley, 1968a; Domcke, 1981). All the data indicate that positrons generally do not form shape resonances or electronic Feshbach resonances in low-energy collisions with molecules. Instead, energy-resolved annihila-

\footnotetext{
${ }^{3}$ The theoretical maximum for the magnitude of $Z_{\text {eff }}$ is given by the unitarity limit of the inelastic cross section (Landau and Lifshitz, 1977), $Z_{\text {eff }} \lesssim 10^{7}$ for room-temperature positrons.
} 

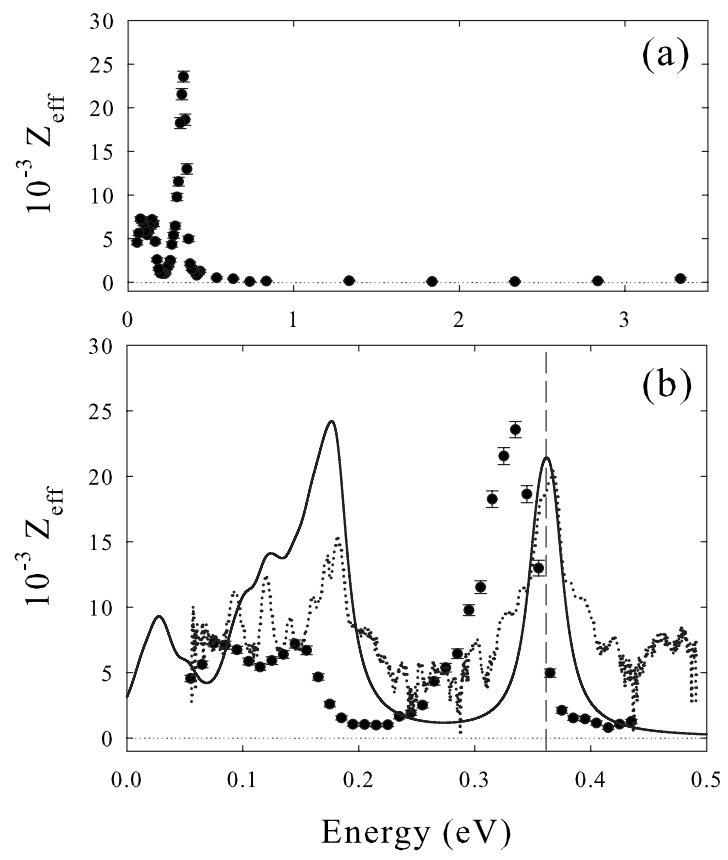

FIG. 1. The normalized annihilation rate $Z_{\text {eff }}(\varepsilon)$ for butane $\mathrm{C}_{4} \mathrm{H}_{10}(-)$ as a function of the total incident positron energy $\varepsilon$ : (a) up to the Ps formation threshold, $E_{\mathrm{th}}=3.8 \mathrm{eV}$, and (b) in the region of the molecular vibrations; dotted curve, the infrared absorption spectrum (Linstrom and Mallard, 2005) (logarithmic vertical scale, arbitrary units); solid curve, the vibrational mode density (in arbitrary units), with the modes represented by Lorentzians with an arbitrary FWHM of $10 \mathrm{meV}$; dashed line, mean energy of the $\mathrm{C}-\mathrm{H}$ stretch fundamentals.

tion studies point to the important role played by the VFR.

These vibrational (or "nuclear-excited") Feshbach resonances involve coupling of the electronic and the nuclear motion beyond the Born-Oppenheimer approximation. It cannot be described by potential-energy surfaces. This type of resonances was originally introduced by Bardsley (1968b) as an "indirect" mechanism for dissociative electron recombination and described using Breit-Wigner theory. In the case of electrons, these VFRs lead to large attachment cross sections which typically reach their maximum values at thermal electron energies (Christophorou et al., 1984). They are also responsible for the formation of long-lived parent negative ions for many complex polyatomic molecules.

Referring to Fig. 1, the energy of the VFR corresponding to mode $\nu$ is given by energy conservation,

$$
\varepsilon_{\nu}=\omega_{\nu}-\varepsilon_{b},
$$

where $\varepsilon_{b}$ is the positron-molecule binding energy and $\omega_{\nu}$ is the vibrational mode energy. The positron binding energy (i.e., the positron affinity) can be measured by the downshift of the resonances from the energies of the vibrational modes. In Fig. 1 for butane, this is most easily seen as the shift in the $\mathrm{C}-\mathrm{H}$ stretch vibrational resonance. The corresponding peak in $Z_{\text {eff }}$ occurs at $330 \mathrm{meV}$, as compared with the vibrational mode frequency of $365 \mathrm{meV}$, indicating that $\varepsilon_{b}=35 \mathrm{meV}$. The
TABLE I. Annihilation rates $Z_{\text {eff }}$ and binding energies $\varepsilon_{b}$ for selected molecules.

\begin{tabular}{lcccr}
\hline \hline Class & & \multicolumn{3}{c}{$\varepsilon_{b}$} \\
$(\mathrm{meV})^{\mathrm{a}}$ & \multicolumn{1}{c}{$Z_{\text {eff }}{ }^{\mathrm{a}}$} \\
\hline Small inorganics & $\mathrm{H}_{2} \mathrm{O}$ & 10 & $<0$ & $170^{\mathrm{b}}$ \\
& $\mathrm{NH}_{3}$ & 9 & $>0$ & $300^{\mathrm{b}}$ \\
Methyl halides & $\mathrm{CH}_{3} \mathrm{~F}$ & 18 & $>0$ & $250^{\mathrm{b}}$ \\
& $\mathrm{CH}_{3} \mathrm{Br}$ & 44 & 40 & $2000^{\mathrm{b}}$ \\
Alkanes & $\mathrm{CH}_{4}$ & 10 & $<0$ & $70^{\mathrm{b}}$ \\
& $\mathrm{C}_{2} \mathrm{H}_{6}$ & 18 & $>0$ & $900^{\mathrm{c}}$ \\
& $\mathrm{C}_{3} \mathrm{H}_{8}$ & 26 & 10 & $10500^{\mathrm{c}}$ \\
& $\mathrm{C}_{6} \mathrm{H}_{14}$ & 50 & 80 & $184000^{\mathrm{c}}$ \\
& $\mathrm{C}_{12} \mathrm{H}_{26}$ & 98 & 220 & $9800000^{\mathrm{c}}$ \\
Alcohols & $\mathrm{CH}_{3} \mathrm{OH}$ & 18 & $>0$ & $750^{\mathrm{b}}$ \\
& $\mathrm{C}_{2} \mathrm{H}_{5} \mathrm{OH}$ & 26 & 45 & $4500^{\mathrm{b}}$ \\
Aromatics & $\mathrm{C}_{6} \mathrm{H}_{6}$ & 42 & 150 & $47000^{\mathrm{c}}$ \\
& $\mathrm{C}_{10} \mathrm{H}_{8}$ & 68 & 300 & $1240000^{\mathrm{c}}$ \\
\hline
\end{tabular}

${ }^{a}$ Values from energy-resolved measurements (Young and Surko, 2008b, 2008c); typical uncertainties in $Z_{\text {eff }}$ and $\varepsilon_{b}$ are $\pm 20 \%$ and $\pm 10 \mathrm{meV}$, respectively.

${ }^{\mathrm{b}}$ Maximum values for positron energies $\varepsilon \geqslant 50 \mathrm{meV}$.

${ }^{c}$ Values of $Z_{\text {eff }}$ at the $\mathrm{C}-\mathrm{H}$ resonance peak.

resonances at lower energies are due to $\mathrm{C}-\mathrm{C}$ modes and $\mathrm{C}-\mathrm{H}$ bend modes and exhibit the same downshift.

There are a number of important chemical trends associated with resonant annihilation on molecules (Murphy and Surko, 1991; Iwata et al., 1995; Young and Surko, 2008b, 2008c). Examples are shown in Table I. Very small molecules, such as $\mathrm{CO}_{2}, \mathrm{CH}_{4}$, or $\mathrm{H}_{2} \mathrm{O}$, have relatively small values of $Z_{\text {eff }}$ (e.g., $Z_{\text {eff }} / Z \lesssim 10$ ), and typically they do not exhibit resonant annihilation peaks. Positrons either do not bind to these species (i.e., $\varepsilon_{b}$ $<0$ ) or they bind extremely weakly. With the exception of methane, all of the alkanes exhibit VFRs, with values of $\varepsilon_{b}$ increasing linearly with the number of carbon atoms $n$, and the magnitudes of $Z_{\text {eff }}$ increasing approximately exponentially with $n$. Most hydrocarbons, including aromatic molecules, alkenes, and alcohols, exhibit similar resonant annihilation spectra.

Much progress has been made in the theoretical understanding of resonant positron annihilation in molecules (Gribakin, 2000, 2001; Gribakin and Gill, 2004). A quantitative theory has been developed for the case of isolated resonances of IR-active vibrational modes, such as those observed in experiments for selected small molecules. The prototypical example is that of the methyl halides, $\mathrm{CH}_{3} X$, where $X$ is a $\mathrm{F}, \mathrm{Cl}$, or $\mathrm{Br}$ atom. Positron coupling to the IR-active modes is evaluated in the dipole approximation using data from IR absorption measurements. The only free parameter in the theory is the positron binding energy, which can be taken from experiment. This yields theoretical annihilation spectra for methyl halides that are in good agreement with the measurements (Gribakin and Lee, 2006a).

A more stringent test of the theory relies on the fact 
that positron binding energies are expected to change little upon isotope substitution. For deuteration this was confirmed experimentally. The binding energies measured for $\mathrm{CH}_{3} \mathrm{Cl}$ and $\mathrm{CH}_{3} \mathrm{Br}$ were used to predict $Z_{\text {eff }}$ for their deuterated analogs. The result is excellent agreement between theory and experiment with no adjustable parameters (Young et al., 2008). In other small molecules, such as ethylene and methanol, IR-inactive modes and multimode vibrations are prominent and must be included to explain the observations (Young et al., 2008).

This theoretical approach explains $Z_{\text {eff }}$ for small polyatomics in which the positron coupling to the modebased VFR and, possibly a few overtones, can be estimated (e.g., when they have dipole coupling). Their $Z_{\text {eff }}$ values are between a few hundred and a few thousand. ${ }^{4}$ However, larger molecules with more than one or two carbons have values of $Z_{\text {eff }}$ that cannot be explained by this theory (cf. Fig. 1 for butane). The current physical picture ascribes their large annihilation rates to large densities of vibrational resonances, known as "dark states" (Gribakin, 2000, 2001), that are not coupled directly to the positron continuum. The positron first attaches to the molecule via a vibrational "doorway state" (e.g., a dipole-allowed mode-based VFR) (Gribakin and Gill, 2004). The vibrational energy is then transferred to the dark states in a process known as intramolecular vibrational energy redistribution (IVR). Such IVR is important for many physical and chemical processes in molecules, including dissociative attachment (Uzer and Miller, 1991; Nesbitt and Field, 1996).

The magnitudes of resonant contributions to $Z_{\text {eff }}$ exhibit a relatively weak dependence on $\varepsilon_{b}$ and on the incident positron energy $\varepsilon$. It is of the form $g=\sqrt{\varepsilon_{b} / \varepsilon}$ and follows from rather general theoretical considerations. When this dependence is factored out, it is found experimentally that the resulting quantity $Z_{\text {eff }} / g$ scales as $\sim N^{4}$, where $N$ is the number of atoms in the molecule. This dependence on $N$ is thought to reflect the rapid increase in the density of the molecular vibrational spectrum with the number of vibrational modes. This dependence is interpreted as evidence that IVR does indeed play an important role in the annihilation process.

Estimates of $Z_{\text {eff }}$ in large molecules, which assume that the IVR process is complete and all modes are populated statistically, predict $Z_{\text {eff }}$ values far in excess of those that are observed. Such estimates also fail to reproduce the energy dependence of $Z_{\text {eff }}$, which is largely determined by the mode-based vibrational doorways. One hypothesis, as yet unconfirmed, is that the IVR process does not run to "completion." It appears that selective coupling of multimode vibrations leaves a large portion of them inactive. The calculation of $Z_{\text {eff }}$ then requires a detailed knowledge of the vibrational mode

\footnotetext{
${ }^{4}$ The heights of resonant peaks in the measured $Z_{\text {eff }}$ spectra are related to the energy spread $\delta \varepsilon$ of the incident positron beam. The value of $Z_{\text {eff }} \sim 10^{3}$ corresponds to the typical $\delta \varepsilon$ $\sim 40 \mathrm{meV}$ used to date (see Sec. III).
}

couplings, and this has not yet been done.

The energy-resolved annihilation experiments provide measures of positron-molecule binding energies, either directly using Eq. (3) or, for very weakly bound states, indirectly through the dependence of $Z_{\text {eff }}$ on $g$. To date, binding energies for about 30 molecules have been measured. They range from $\sim 1 \mathrm{meV}$ in small molecules, such as $\mathrm{CH}_{3} \mathrm{~F}$, to $\sim 300 \mathrm{meV}$ for large alkanes (Young and Surko, 2008b, 2008c). A recent analysis indicates that these binding energies increase approximately linearly with the molecular dipole polarizability and dipole moment and, for aromatic molecules, the number of $\pi$ bonds (Danielson et al., 2009).

For atoms, comparatively accurate positron binding energies have been predicted theoretically for about ten species (Mitroy et al., 2002), but there are no measurements. There have been a number of calculations for positron binding to molecules (Schrader and Wang, 1976; Kurtz and Jordan, 1978, 1981; Danby and Tennyson, 1988; Bressanini et al., 1998; Strasburger, 1999, 2004; Schrader and Moxom, 2001; Tachikawa et al., 2003; Buenker et al., 2005; Chojnacki and Strasburger, 2006; Gianturco et al., 2006; Buenker and Liebermann, 2008; Carey et al., 2008). Most of these molecules have large dipole moments which facilitate binding. In contrast, most molecules for which the binding energies are known from experiment are either nonpolar or only weakly polar. Thus at present, there are almost no species for which experiment and theory can be compared, and so this is a critical area for future research.

Presented here is a review of theoretical and experimental results for positron annihilation on molecules in the range of energies below the positronium formation threshold. Emphasis is placed upon the case in which positrons bind to the target and annihilation proceeds via the formation of vibrational Feshbach resonances. Current knowledge of positron-molecule binding energies, obtained from both experiment and theoretical calculations, is summarized. These results are related to studies of positron-induced fragmentation of molecules, annihilation gamma-ray spectra, annihilation in dense gases where nonlinear effects are observed, and to analogous electron interactions with molecules and clusters.

\section{THEORY}

\section{A. Annihilation basics}

The process of electron-positron annihilation is described by quantum electrodynamics (QED). In the nonrelativistic Born approximation, the cross section for annihilation into two photons averaged over the electron and positron spins is (Berestetskii et al., 1982)

$$
\bar{\sigma}_{2 \gamma}=\pi r_{0}^{2} c / v,
$$

where $v$ is the relative velocity of the two particles. This cross section obeys the $1 / v$ threshold law which describes inelastic collisions with fast particles in the final state (Landau and Lifshitz, 1977). 
The two-photon annihilation described by Eq. (4) is allowed only when the total spin $S$ of the electronpositron pair is zero. For $S=1$ the smallest possible number of annihilation photons is three. The corresponding spin-averaged cross section is (Berestetskii et al., 1982)

$$
\bar{\sigma}_{3 \gamma}=\frac{4}{3}\left(\pi^{2}-9\right) \alpha r_{0}^{2} c / v
$$

where $\alpha=e^{2} / \hbar c$ (in cgs units) is the fine structure constant, $\alpha \approx 1 / 137$. Since $\bar{\sigma}_{3 \gamma}$ is 400 times smaller than $\bar{\sigma}_{2 \gamma}$, positron annihilation in many-electron systems is dominated by the two-gamma process.

Numerically, the cross section in Eq. (4) is $\bar{\sigma}_{2 \gamma}$ $\sim 10^{-8} \mathrm{c} / v$ a.u. ${ }^{5}$ Hence the annihilation rate is usually much smaller than the rates for other atomic collision processes, even at low positron velocities (e.g., thermal, $v \sim 0.05$ a.u. at $300 \mathrm{~K}$ ). When a fast positron, such as that emitted in a $\beta^{+}$decay, moves through matter, it loses energy quickly through collisions, first by direct ionization, positronium formation and electronic excitation, and then by vibrational excitation and elastic collisions. As a result, the positrons are typically slow to thermal energies (i.e., $\sim 25 \mathrm{meV}$ for $T=300 \mathrm{~K}$ ) before annihilation.

At small velocities, $v \leqq 1$ a.u., Eq. (4) must be modified to take into account the Coulomb interaction between the electron and positron. The typical momenta exchanged in the annihilation process are $p \sim m c$. The corresponding separation $r \sim \hbar / m c$ is small compared to $a_{0}$, and in the nonrelativistic limit, the annihilation takes place when the electron and positron are at the same point. The cross section in Eq. (4) must then be multiplied by the probability density at the origin (Landau and Lifshitz, 1977),

$$
|\psi(0)|^{2}=\frac{2 \pi}{v\left(1-e^{-2 \pi / v}\right)},
$$

where the wave function $\psi$ is normalized by $\psi(\mathbf{r}) \simeq e^{i \mathbf{k} \cdot \mathbf{r}}$ at $r \gg a_{0}$. This increases the annihilation cross section.

The annihilation cross section for many-electron targets is traditionally written as (Pomeranchuk, 1949; Fraser, 1968)

$$
\sigma_{a}=\bar{\sigma}_{2 \gamma} Z_{\mathrm{eff}}=\pi r_{0}^{2} \frac{c}{v} Z_{\mathrm{eff}},
$$

where $Z_{\text {eff }}$ represents the effective number of electrons that contribute to the annihilation. In the Born approximation, $Z_{\text {eff }}=Z$, the total number of target electrons.

At small positron energies (e.g., $\varepsilon \lesssim 1 \mathrm{eV}$ ), however, $Z_{\text {eff }}$ can be different from $Z$. First, there is a strong repulsion between the positron and the atomic nuclei. This prevents the positron from penetrating deep into the atoms so that the annihilation involves predominantly electrons in the valence and near-valence subshells, thereby reducing $Z_{\text {eff }}$. On the other hand, the positron is

\footnotetext{
${ }^{5}$ We make use of atomic units (a.u.), in which $m=|e|=\hbar=1$, $c=\alpha^{-1} \approx 137$ a.u., and the Bohr radius $a_{0}=\hbar^{2} / m e^{2}$ (in cgs units) also equals unity.
}

attracted to the target by a long-range polarization potential $-\alpha_{d} / 2 r^{4}$, where $\alpha_{d}$ is the target dipole polarizability, which enhances $Z_{\text {eff. }}$ There is also a short-range enhancement of $Z_{\text {eff }}$ due to the Coulomb interaction between the annihilating electron and positron, which has the same origin as the expression in Eq. (6). Finally, if the target binds the positron, the annihilation can be enhanced by positron capture into this bound state. The cross section for radiative capture (i.e., by emission of a photon) is small, namely, $\sigma_{c} \sim \alpha^{3} a_{0}^{2}$ (Berestetskii et al., 1982). In collisions with molecules, the positron can transfer its energy to vibrations forming a positronmolecule complex. This process is effective in enhancing the annihilation rate. It is the principal focus of the present review.

As follows from its definition by Eq. (7), $Z_{\text {eff }}$ is equal to the electron density at the positron,

$$
Z_{\mathrm{eff}}=\int \sum_{i=1}^{Z} \delta\left(\mathbf{r}-\mathbf{r}_{i}\right)\left|\Psi_{\mathbf{k}}\left(\mathbf{r}_{1}, \ldots, \mathbf{r}_{Z}, \mathbf{r}\right)\right|^{2} d \mathbf{r}_{1} \cdots d \mathbf{r}_{Z} d \mathbf{r}
$$

where $\Psi_{\mathbf{k}}\left(\mathbf{r}_{1}, \ldots, \mathbf{r}_{Z}, \mathbf{r}\right)$ is the total wave function of the system, with electron coordinates $\mathbf{r}_{i}$ and positron coordinate $\mathbf{r}$. This wave function describes the scattering of the positron with initial momentum $\mathbf{k}$ by the atomic or molecular target and is normalized to the positron plane wave at large separations,

$$
\Psi_{\mathbf{k}}\left(\mathbf{r}_{1}, \ldots, \mathbf{r}_{Z}, \mathbf{r}\right) \simeq \Phi_{0}\left(\mathbf{r}_{1}, \ldots, \mathbf{r}_{Z}\right) e^{i \mathbf{k} \cdot \mathbf{r}}
$$

where $\Phi_{0}$ is the wave function of the initial (e.g., ground) state of the target. For molecules, both $\Psi_{\mathbf{k}}$ and $\Phi_{0}$ also depend on the nuclear coordinates, which must be integrated over in Eq. (8).

Equations (7) and (8) determine the annihilation rate in binary positron-molecule collisions,

$$
\lambda=\sigma_{a} v n=\pi r_{0}^{2} c Z_{\mathrm{eff}} n,
$$

where $n$ is the gas number density. To compare with experiment, this rate is averaged over the positron energy distribution. For thermal positrons this distribution is a Maxwellian, while in beam experiments, it is determined by the parameters of the beam. Empirically Eq. (10) is also used to describe experiments at high densities where $Z_{\text {eff }}$ becomes density dependent (see Sec. VIII.C).

\section{B. Gamma-ray spectra and annihilation rates}

In the nonrelativistic limit, the two-photon QED annihilation amplitude can be expressed in terms of an effective annihilation operator 


$$
\hat{O}_{a}(\mathbf{P}) \equiv \int e^{-i \mathbf{P} \cdot \mathbf{r}} \hat{\psi}(\mathbf{r}) \hat{\varphi}(\mathbf{r}) d \mathbf{r}
$$

where $\hat{\psi}(\mathbf{r})$ and $\hat{\varphi}(\mathbf{r})$ are the electron and positron destruction operators ${ }^{6}$ and $\mathbf{P}$ is the total momentum of the photons (Ferrell, 1956; Lee, 1957; Dunlop and Gribakin, 2006). The probability distribution of $\mathbf{P}$ in an annihilation event is given by

$$
W_{f}(\mathbf{P})=\pi r_{0}^{2} c\left|\left\langle f\left|\hat{O}_{a}(\mathbf{P})\right| i\right\rangle\right|^{2},
$$

where $|i\rangle$ is the initial state with $Z$ electrons and the positron (e.g., that with the wave function $\Psi_{\mathbf{k}}$ ) and $|f\rangle$ is the state of $Z-1$ electrons after the annihilation.

For $\mathbf{P}=0$, the two photons are emitted in opposite directions and have equal energies, $E_{\gamma 1}=E_{\gamma 2} \equiv E_{\gamma} \approx m c^{2}$. For $\mathbf{P} \neq 0$ the photon energy is Doppler shifted, e.g.,

$$
E_{\gamma 1}=E_{\gamma}+m c|\mathbf{V}| \cos \theta,
$$

where $\mathbf{V}=\mathbf{P} / 2 m$ is the center-of-mass velocity of the electron-positron pair and $\theta$ is the angle between $\mathbf{V}$ and the direction of the photon. Averaging the distribution of the Doppler shifts $\epsilon=E_{\gamma 1}-E_{\gamma}=(P c / 2) \cos \theta$, over the direction of emission of the photons, gives the photon energy spectrum

$$
w_{f}(\boldsymbol{\epsilon})=\frac{1}{c} \iint_{2 \mid \epsilon / c}^{\infty} W_{f}(\mathbf{P}) \frac{P d P d \Omega_{\mathbf{P}}}{(2 \pi)^{3}} .
$$

In Cartesian coordinates,

$$
w_{f}(\epsilon)=\frac{2}{c} \iint W_{f}\left(P_{x}, P_{y}, 2 \epsilon / c\right) \frac{d P_{x} d P_{y}}{(2 \pi)^{3}} .
$$

This form shows that the energy spectrum is proportional to the probability density for a component of $\mathbf{P}$. This quantity can be measured either by sampling the Doppler spectrum of the gamma rays or by measuring the angular deviation of the two photons (see Sec. III.F).

When a low-energy positron annihilates with a bound electron with energy $\varepsilon_{n}$, the mean photon energy $E_{\gamma}$ is shifted by $\varepsilon_{n} / 2$ relative to $m c^{2}$. This shift is much smaller than the typical Doppler shift $\epsilon$ due to the momentum of the bound electron $P \sim \sqrt{2 m\left|\varepsilon_{n}\right|}$, which corresponds to $\epsilon \sim P c \sim \sqrt{\left|\varepsilon_{n}\right| m c^{2}} \gg\left|\varepsilon_{n}\right|$. The resulting width and shape of the gamma spectrum contain important information about the bound electrons.

In most experiments, the annihilation photons are not detected in coincidence with the final state $f$, and the observed spectrum is the sum over all final states $w(\epsilon)$ $=\Sigma_{f} w_{f}(\epsilon)$. However, this spectrum still reveals contribu-

\footnotetext{
${ }^{6}$ In Eq. (11) the spin indices in $\hat{\psi}(\mathbf{r})$ and $\hat{\varphi}(\mathbf{r})$ are suppressed, and summation over them is assumed. This form can be used in systems with paired electron spins or when averaging over the positron spin. The modulus-squared amplitude is then multiplied by the spin-averaged QED factor $\pi r_{0}^{2} c$. In general, one should use the spin-singlet combination of the annihilation operators in Eq. $(11),(1 / \sqrt{2})\left(\hat{\psi}_{\uparrow} \hat{\varphi}_{\downarrow}-\hat{\psi}_{\downarrow} \hat{\varphi}_{\uparrow}\right)$, together with the twophoton annihilation factor $4 \pi r_{0}^{2} c$.
}

tions of different final states. For example, in partially fluorinated alkanes, annihilation with the tightly bound fluorine $2 p$ electrons results in a broader spectral component than annihilation with the more diffuse $\mathrm{C}-\mathrm{H}$ bond electrons. This allows one to deduce the relative fraction of the corresponding annihilation events (Iwata et al., 1997); see Sec. VIII.A.

The total annihilation rate in the state $i$ leading to the final state $f$ is obtained by integration over the momenta

$$
\lambda_{f}=\pi r_{0}^{2} c \int\left|\left\langle f\left|\hat{O}_{a}(\mathbf{P})\right| i\right\rangle\right|^{2} \frac{d^{3} P}{(2 \pi)^{3}},
$$

and the total annihilation rate in state $i$ is

$$
\lambda=\sum_{f} \lambda_{f}=\pi r_{0}^{2} c \int\left\langle i\left|\hat{n}_{-}(\mathbf{r}) \hat{n}_{+}(\mathbf{r})\right| i\right\rangle d \mathbf{r},
$$

where $\hat{n}_{-}(\mathbf{r})=\hat{\psi}^{\dagger}(\mathbf{r}) \hat{\psi}(\mathbf{r})$ and $\hat{n}_{+}(\mathbf{r})=\hat{\varphi}^{\dagger}(\mathbf{r}) \hat{\varphi}(\mathbf{r})$ are the electron and positron density operators. The annihilation rate is thus given by the expectation value of the electron density at the positron. Equation (17) gives the twophoton annihilation rate in a system of one positron and one target atom or molecule. For a positron moving through a gas of density $n$, the annihilation rate takes the form of Eq. (10). Normalizing the initial state $i$ to one positron per unit volume, as $\Psi_{\mathbf{k}}$ in Eq. (9), one obtains

$$
Z_{\text {eff }}=\int\left\langle i\left|\hat{n}_{-}(\mathbf{r}) \hat{n}_{+}(\mathbf{r})\right| i\right\rangle d \mathbf{r} .
$$

In the coordinate representation, this yields Eq. (8).

In the independent-particle approximation, the electronic parts of the initial and final states are Slater determinants constructed from the electron orbitals (e.g., in the Hartree-Fock scheme). The incident positron is described by its own wave function $\varphi_{\mathbf{k}}(\mathbf{r})$, and the annihilation amplitude $\left\langle f\left|\hat{O}_{a}(\mathbf{P})\right| i\right\rangle$ takes the form

$$
A_{n \mathbf{k}}(\mathbf{P})=\int e^{-i \mathbf{P} \cdot \mathbf{r}} \psi_{n}(\mathbf{r}) \varphi_{\mathbf{k}}(\mathbf{r}) d \mathbf{r},
$$

where $\psi_{n}(\mathbf{r})$ is the orbital of the annihilated electron. In this approximation

$$
Z_{\text {eff }}=\sum_{n=1}^{Z} \int\left|\psi_{n}(\mathbf{r})\right|^{2}\left|\varphi_{\mathbf{k}}(\mathbf{r})\right|^{2} d \mathbf{r},
$$

i.e., the average product of the electron and positron densities.

\section{Positron-molecule wave function}

The wave function $\Psi_{\mathbf{k}}$ for the positron colliding with a molecule can be written as (Gribakin, 2000, 2001)

$$
\Psi_{\mathbf{k}}=\Psi_{\mathbf{k}}^{(0)}+\sum_{\nu} \frac{\Psi_{\nu}\left\langle\Psi_{\nu}|V| \Psi_{\mathbf{k}}^{(0)}\right\rangle}{\varepsilon-\varepsilon_{\nu}+(i / 2) \Gamma_{\nu}} .
$$

The first term on the right-hand side describes direct or potential scattering of the positron by the target. The 
corresponding wave function $\Psi_{\mathbf{k}}^{(0)}$ is determined by the positron interaction with the charge distribution of the ground-state target and electron-positron correlation effects (e.g., target polarization and virtual Ps formation). It neglects the coupling $V$ between the electron-positron and nuclear (vibrational) degrees of freedom. The second term describes positron capture into the vibrational Feshbach resonances. It is present for molecules that can bind the positron. These resonances correspond to vibrationally excited states $\Psi_{\nu}$ of the positron-molecule complex, embedded in the positron continuum. They occur when the positron energy $\varepsilon=k^{2} / 2$ is close to $\varepsilon_{\nu}=E_{\nu}-\varepsilon_{b}$, where $\varepsilon_{b}$ is the positron binding energy and $E_{\nu}$ is the vibrational excitation energy of the positronmolecule complex. Equation (21) has the appearance of a standard perturbation-theory formula, but the energies of the positron-molecule quasibound states $\Psi_{\nu}$ in the denominator are complex, $\varepsilon_{\nu}-(i / 2) \Gamma_{\nu}$, where $\Gamma_{\nu}=\Gamma_{\nu}^{a}+\Gamma_{\nu}^{e}+\Gamma_{\nu}^{i}$ is the total width of the resonance. In atomic units $\Gamma_{\nu}$ is equal to the decay rate of the resonant state. It contains contributions of positron annihilation and elastic escape $\Gamma_{\nu}^{a}$ and $\Gamma_{\nu}^{e}$, respectively, and possibly also the inelastic escape rate $\Gamma_{\nu}^{i}$. The latter describes positron autodetachment accompanied by vibrational transitions to the states other than the initial state.

Molecular rotations are, in general, not expected to affect positron annihilation. The rotational motion is slow compared to the motion of the positron or the vibrational motion. Accordingly, direct scattering can be considered for fixed molecular orientation and the results averaged over the orientations. Positron capture in VFRs at low energies is dominated by the $s$ wave or at most a few lower partial waves. Hence in the capture process, the angular momentum of the molecule remains unchanged or changes little.

The positron capture amplitude $\left\langle\Psi_{\nu}|V| \Psi_{\mathbf{k}}^{(0)}\right\rangle$ determines the elastic width in state $\nu$,

$$
\Gamma_{\nu}^{e}=2 \pi \int\left|\left\langle\Psi_{\nu}|V| \Psi_{\mathbf{k}}^{(0)}\right\rangle\right|^{2} \frac{k d \Omega_{\mathbf{k}}}{(2 \pi)^{3}} .
$$

If the positron interaction with the vibrations cannot be described by perturbation theory, Eqs. (21) and (22) remain valid provided the amplitudes $\left\langle\Psi_{\nu}|V| \Psi_{\mathbf{k}}^{(0)}\right\rangle$ are replaced by their nonperturbative values.

According to Eq. (17), the annihilation rate of the positron-molecule state $\Psi_{\nu}$ is given by

$$
\Gamma_{\nu}^{a}=\pi r_{0}^{2} c \rho_{e p},
$$

where $\rho_{e p}$ is the average electron density at the positron,

$$
\rho_{e p}=\int \sum_{i=1}^{Z} \delta\left(\mathbf{r}-\mathbf{r}_{i}\right)\left|\Psi_{\nu}\left(\mathbf{r}_{1}, \ldots, \mathbf{r}_{Z}, \mathbf{r}\right)\right|^{2} d \mathbf{r}_{1} \cdots d \mathbf{r}_{Z} d \mathbf{r},
$$

with the integration extending to the nuclear coordinates in the wave function $\Psi_{\nu}$. The amplitude of the nuclear motion is small, and $\rho_{e p}$ is expected to depend weakly on the degree of vibrational excitation in state $\nu$.
To calculate $Z_{\text {eff }}$, the wave function from Eq. (21) is substituted into Eq. (8), which yields

$$
\begin{aligned}
Z_{\text {eff }}= & \left\langle\Psi_{\mathbf{k}}\left|\sum_{i=1}^{Z} \delta\left(\mathbf{r}-\mathbf{r}_{i}\right)\right| \Psi_{\mathbf{k}}\right\rangle \\
= & \left\langle\Psi_{\mathbf{k}}^{(0)}\left|\sum_{i=1}^{Z} \delta\left(\mathbf{r}-\mathbf{r}_{i}\right)\right| \Psi_{\mathbf{k}}^{(0)}\right\rangle+\left\{\begin{array}{c}
\text { interference } \\
\text { terms }
\end{array}\right\} \\
& +\frac{2 \pi^{2}}{k} \sum_{\mu \nu} \frac{A_{\mu}^{*}\left\langle\Psi_{\mu}\left|\sum_{i=1}^{Z} \delta\left(\mathbf{r}-\mathbf{r}_{i}\right)\right| \Psi_{\nu}\right\rangle A_{\nu}}{\left(\varepsilon-\varepsilon_{\mu}-\frac{i}{2} \Gamma_{\mu}\right)\left(\varepsilon-\varepsilon_{\nu}+\frac{i}{2} \Gamma_{\nu}\right)},
\end{aligned}
$$

where the capture amplitude $A_{\nu}$ is related to the elastic width by $\Gamma_{\nu}^{e}=2 \pi\left|A_{\nu}\right|^{2}$. The terms on the right-hand side describe the contributions of direct and resonant annihilation and the interference between the two. We now examine the two main contributions in detail.

The separation of the wave function into the direct and resonant parts in Eq. (21) is valid because the positron VFRs are narrow. This is a consequence of the weakness of coupling between the positron and the vibrational motion (i.e., small capture widths $\Gamma_{v}^{e}$, see Sec. II.F). In spite of this, the resonant contribution to the annihilation rate for complex polyatomics exceeds the direct contributions by orders of magnitude.

\section{Direct annihilation: Virtual and weakly bound positron states}

The potential scattering wave function $\Psi_{\mathbf{k}}^{(0)}$ satisfies the Schrödinger equation

$$
\left(T+U-E_{0}\right) \Psi_{\mathbf{k}}^{(0)}=\varepsilon \Psi_{\mathbf{k}}^{(0)},
$$

where $T$ is the kinetic energy operator for the electrons and positron, $U$ is the sum of all Coulomb interactions between the particles (with the nuclei at their equilibrium positions), and $E_{0}$ is the target ground-state energy.

For positron energies below the Ps-formation threshold, annihilation occurs when the positron is within the range of the target ground-state electron cloud. At such distances, the interaction $U$ between the particles is much greater than the positron energy $\varepsilon$. Therefore, the $\varepsilon \Psi_{\mathbf{k}}^{(0)}$ term in Eq. (26) can be neglected, and the solution $\Psi_{\mathbf{k}}^{(0)}$ at these small separations does not depend on $\varepsilon$, except through a normalization factor.

When the positron is outside the target, $\Psi_{\mathbf{k}}^{(0)}$ contains contributions of the incident and scattered positron waves,

$$
\Psi_{\mathbf{k}}^{(0)}\left(\mathbf{r}_{1}, \ldots, \mathbf{r}_{Z}, \mathbf{r}\right) \simeq \Phi_{0}\left(\mathbf{r}_{1}, \ldots, \mathbf{r}_{Z}\right)\left[e^{i \mathbf{k} \cdot \mathbf{r}}+f_{\mathbf{k} \mathbf{k}^{\prime}} \frac{e^{i k r}}{r}\right]
$$

where $f_{\mathbf{k k}^{\prime}}$ is the scattering amplitude and $\mathbf{k}^{\prime}=k \mathbf{r} / r$. Inside the target, $\Psi_{\mathbf{k}}^{(0)}$ is determined by matching it with Eq. (27) at the target boundary $r=R$, where $R$ is the characteristic radius of the target. For small positron momenta $k R \ll 1$, the scattering is dominated by the $s$ 
wave, and the amplitude $f_{\mathbf{k k}^{\prime}}$ can be replaced by the $s$-wave amplitude $f_{0}$. As a result, the integrand in Eq. (8) for $Z_{\text {eff }}$ is proportional to $\left|1+f_{0} / R\right|^{2}$ (Dzuba et al., 1993). This gives the following estimate for $Z_{\text {eff }}$ due to direct annihilation (Gribakin, 2000),

$$
Z_{\mathrm{eff}}^{(\mathrm{dir})} \simeq 4 \pi \rho_{e} \delta R\left(R^{2}+2 R \operatorname{Re} f_{0}+\sigma_{\mathrm{el}} / 4 \pi\right),
$$

where $\rho_{e}$ is the effective electron density in the region of annihilation, $\delta R$ is the range of distances where the positron annihilates, and $\sigma_{\mathrm{el}}$ is the elastic cross section. At small positron energies, $\sigma_{\mathrm{el}} \simeq 4 \pi\left|f_{0}\right|^{2}$, and in the zeroenergy limit $\sigma_{\mathrm{el}}=4 \pi a^{2}$, where $a$ is the positron scattering length, $a=-f_{0}$ at $k=0$. $^{7}$

A simple estimate of the factor $4 \pi \rho_{e} \delta R \equiv F$ in Eq. (28) is obtained using the Ps density at the origin, $\rho_{e} \sim \rho_{\mathrm{Ps}}$ $=1 / 8 \pi$, and $\delta R \sim 1$, which yields $F \sim 0.5$. Equation (28) then shows that the magnitude of $Z_{\text {eff }}^{(\mathrm{dir})}$ is comparable to the geometrical cross section of the target (in atomic units), unless $\sigma_{\mathrm{el}}$ is much greater than $R^{2}$.

When the scattering cross section is large, the annihilation rate is greatly enhanced. This occurs when the positron has a virtual or a bound state close to zero energy (Goldanskii and Sayasov, 1964). Such states are characterized by a small parameter $\kappa=1 / a,|\kappa| \ll R^{-1}$. It is related to the energy of the bound state $\varepsilon_{0}=-\kappa^{2} / 2$ (for $\kappa>0$ ) or virtual state $\varepsilon_{0}=\kappa^{2} / 2$ (for $\kappa<0$ ). This parameter determines the low-energy $s$-wave scattering amplitude $f_{0}=-(\kappa+i k)^{-1}$ and cross section $\sigma_{\text {el }} \simeq 4 \pi /\left(\kappa^{2}+k^{2}\right)$ (Landau and Lifshitz, 1977). For small $\kappa$, this cross section can be much greater than the geometrical size of the target. The last term in brackets in Eq. (28) then dominates, and $Z_{\text {eff }}^{(\text {dir) }}$ shows a similar enhancement (Dzuba et al., 1993; Mitroy and Ivanov, 2002) ${ }^{8}$

$$
Z_{\mathrm{eff}}^{(\mathrm{dir})} \simeq \frac{F}{\kappa^{2}+k^{2}}
$$

The applicability of Eq. (29) is shown in Fig. 2. It shows the $Z_{\text {eff }}$ values from the Schwinger multichannel (SMC) calculation for $\mathrm{C}_{2} \mathrm{H}_{2}$ and $\mathrm{C}_{2} \mathrm{H}_{4}$ (Varella et al., 2002), fitted using Eq. (29) with a small vertical offset to account for the nonresonant $Z_{\text {eff }}$ background. According to the SMC calculation, both molecules possess virtual positron states. This results in the characteristic rise of $Z_{\text {eff }}$ at small positron momenta described by Eq. (29). The virtual level in $\mathrm{C}_{2} \mathrm{H}_{2}$ (fitted value $\kappa=0.0041$ ) lies closer to zero energy than in $\mathrm{C}_{2} \mathrm{H}_{4}(\kappa=0.0372)$, which manifests in the large $Z_{\text {eff }}$ values for acetylene. The fitted factor $F \approx 0.25$ for the two molecules is smaller than the estimate obtained from high-quality atomic calculations (see below). This is likely an indication of the lack

\footnotetext{
${ }^{7}$ If the target molecule has a permanent dipole moment $\mu$, the long-range dipole potential $\boldsymbol{\mu} \cdot \mathbf{r} / r^{3}$ dominates the lowenergy scattering (Fabrikant, 1977). This makes $\sigma_{\mathrm{el}}$ infinite, while $Z_{\text {eff }}$ remains finite, making Eq. (28) invalid.

${ }^{8}$ The long-range polarization potential $-\alpha_{d} / 2 r^{4}$ modifies the near-threshold form of $\sigma_{\mathrm{el}}$ and $Z_{\mathrm{eff}}^{(\mathrm{dir})}$ (Gribakin, 2000; Mitroy, 2002), but Eq. (29) can still be used as an estimate.
}

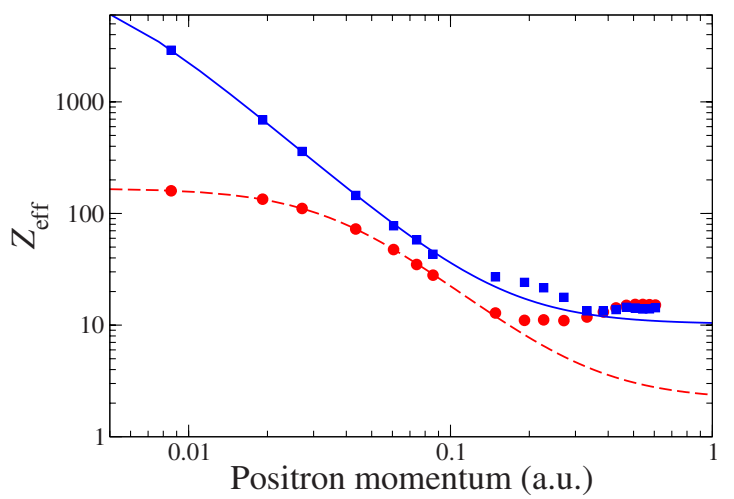

FIG. 2. (Color online) Comparison of the $Z_{\text {eff }}$ values calculated using the SMC method for $\mathrm{C}_{2} \mathrm{H}_{2}$ (squares) and $\mathrm{C}_{2} \mathrm{H}_{4}$ (circles) by Varella et al. (2002) (see Sec. II.H.1) with the fit using Eq. (29) with a constant vertical offset. Parameters of the fit: $\mathrm{C}_{2} \mathrm{H}_{2}$ (solid curve), $F=0.261$, and $\kappa=0.0041 ; \mathrm{C}_{2} \mathrm{H}_{4}$ (dashed curve), $F=0.230$, and $\kappa=0.0372$.

of short-range correlation terms in the SMC calculation, which would enhance the electron density at the positron (see Sec. II.H.1).

Positron virtual states explain the large thermal $Z_{\text {eff }}$ values observed at room temperature in heavier noble gases (Dzuba et al., 1993, 1996). The value of $Z_{\mathrm{eff}}=400$ observed for Xe (Murphy and Surko, 1990) is close to the maximum direct annihilation rate for thermal positrons at $300 \mathrm{~K}$. It is estimated from Eq. (29) to be

$$
Z_{\text {eff }}^{(\text {dir) }} \lesssim 10^{3} .
$$

Higher $Z_{\text {eff }}$ values observed in many polyatomics (see, e.g., Table I) can be understood only by considering positron-molecule binding and resonances.

The annihilation rate for the positron bound to an atom or molecule is

$$
\Gamma^{a}=\pi r_{0}^{2} c \int \sum_{i=1}^{Z} \delta\left(\mathbf{r}-\mathbf{r}_{i}\right)\left|\Psi_{0}\left(\mathbf{r}_{1}, \ldots, \mathbf{r}_{Z}, \mathbf{r}\right)\right|^{2} d \mathbf{r}_{1} \cdots d \mathbf{r}_{Z} d \mathbf{r},
$$

where $\Psi_{0}$ is the wave function of the bound state. For a weakly bound state (e.g., $\left.\varepsilon_{b} \ll 1 \mathrm{eV}\right) \Gamma^{a}$ can be estimated in a way similar to that used for $Z_{\text {eff }}^{(\text {dir) }}$ above. When the positron is outside the target $(r>R), \Psi_{0}$ takes the form

$$
\Psi_{0}\left(\mathbf{r}_{1}, \ldots, \mathbf{r}_{Z}, \mathbf{r}\right) \simeq \Phi_{0}\left(\mathbf{r}_{1}, \ldots, \mathbf{r}_{Z}\right) \frac{A}{r} e^{-\kappa r},
$$

where $A$ is the asymptotic normalization constant. ${ }^{9}$ For weak binding $\left(\kappa \ll R^{-1}\right)$ the main contribution to the normalization integral,

\footnotetext{
${ }^{9}$ Equation (32) assumes that the ionization potential of the atomic system satisfies $E_{i}>E_{\mathrm{Ps}}$. For $E_{i}<E_{\mathrm{Ps}}$ the asymptotic form is that of Ps bound to the positive ion (Mitroy et al., 2002).
} 


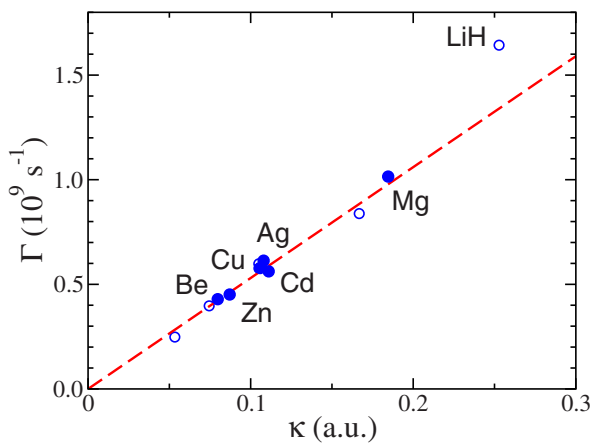

FIG. 3. (Color online) Dependence of the annihilation rate $\Gamma^{a}$ for positron bound states on the parameter $\kappa=\sqrt{2 \varepsilon_{b}}$ : solid circles, recent results for six atoms (Bromley and Mitroy, 2002, 2006, 2010; Mitroy et al., 2002, 2008); open circles, earlier results for these atoms (Mitroy et al., 2002) and LiH molecule (Mitroy and Ryzhikh, 2000); dashed line is the fit $\Gamma^{a}=5.3 \kappa$ (in $10^{9} \mathrm{~s}^{-1}$ ) which corresponds to $F=0.66$ a.u.

$$
\int\left|\Psi_{0}\left(\mathbf{r}_{1}, \ldots, \mathbf{r}_{Z}, \mathbf{r}\right)\right|^{2} d \mathbf{r}_{1} \cdots d \mathbf{r}_{Z} d \mathbf{r}=1
$$

comes from large positron separations where Eq. (32) is valid. This yields

$$
A=\sqrt{\kappa / 2 \pi} \text {. }
$$

By matching the wave function $\Psi_{0}$ in Eq. (31) at $r=R$ with the asymptotic form in Eq. (32), one obtains

$$
\Gamma^{a} \simeq \pi r_{0}^{2} c 4 \pi \rho_{e} \delta R|A|^{2}=\pi r_{0}^{2} c F \frac{\kappa}{2 \pi}
$$

(Gribakin, 2001). Hence the electron-positron contact density from Eq. (24) is estimated by

$$
\rho_{e p} \simeq(F / 2 \pi) \kappa .
$$

Equation (35) shows that $\Gamma^{a}$ is proportional to $\kappa$ $=\sqrt{2 \varepsilon_{b}}$ [i.e., to the square root of the binding energy; see Mitroy and Ivanov (2002) for an alternative derivation]. ${ }^{10}$ This relationship between $\Gamma^{a}$ and $\kappa$ is confirmed by positron-atom bound-state calculations (Mitroy et al., 2002). Figure 3 shows values for six atoms with $E_{i}>E_{\mathrm{Ps}}$, namely, $\mathrm{Be}, \mathrm{Mg}, \mathrm{Cd}, \mathrm{Cu}, \mathrm{Zn}$, and $\mathrm{Ag}$, obtained using high-quality configuration interaction and stochastic variational methods (see Sec. II.H.2 for details). Note that the datum for the $\mathrm{LiH}$ molecule also follows this trend, in spite of its large dipole moment $[\mu=5.9 \mathrm{D}$ (Lide, 2000)] and relatively strong binding. A linear fit through the atomic data points gives a value for the factor $F=4 \pi \rho_{e} \delta R$ in Eq. (35), namely, $F \approx 0.66$ a.u., which is close to the rough estimate given above. One

\footnotetext{
${ }^{10}$ Equation (32) is valid if the positron-target interaction is short range. It must be modified if the molecule has a dipole moment [see, e.g., Fabrikant (1977)]. However, Eq. (35) can be used as an estimate if the dipole force does not play a dominant role in the binding.
}

can use this value to evaluate the annihilation rates for positron-molecule bound states from Eq. (35), provided their binding energies are known.

\section{E. Resonant annihilation}

The effect of resonances on $Z_{\text {eff }}$ is described by the second and third terms in Eq. (25). It is dominated by the diagonal part of the double sum in the last term. The off-diagonal and interference terms vanish upon averaging over the positron energy and can usually be neglected. The resonant contribution to the annihilation cross section is described by the Breit-Wigner formula (Landau and Lifshitz, 1977),

$$
\sigma_{a}=\frac{\pi}{k^{2}} \sum_{\nu} \frac{b_{\nu} \Gamma_{\nu}^{a} \Gamma_{\nu}^{e}}{\left(\varepsilon-\varepsilon_{\nu}\right)^{2}+\frac{1}{4} \Gamma_{\nu}^{2}},
$$

where $b_{\nu}$ is the degeneracy of the $\nu$ th resonance. Equations (7), (23), and (37) then give the resonant $Z_{\text {eff, }}$,

$$
Z_{\mathrm{eff}}^{(\mathrm{res})}=\frac{\pi}{k} \rho_{e p} \sum_{\nu} \frac{b_{\nu} \Gamma_{\nu}^{e}}{\left(\varepsilon-\varepsilon_{\nu}\right)^{2}+\frac{1}{4} \Gamma_{\nu}^{2}} .
$$

The contact density $\rho_{e p}$ can be estimated from Eq. (36) if the positron binding energy is known. To calculate $Z_{\mathrm{eff}}^{(\mathrm{res})}$, one also needs the energies and widths of the resonances. The former are determined by the positron binding energy and the vibrational excitation energies of the positron-molecule complex. The elastic and total rates depend on the strength of coupling between the positron and the vibrational motion and, for overtones and combination excitations, on the strength of anharmonic terms in the vibrational Hamiltonian. This makes an $a b$ initio calculation of resonant $Z_{\text {eff }}$ a multifaceted problem.

\section{F. Resonances due to infrared-active modes}

One case in which such a calculation is possible is that of isolated vibrational resonances of IR-active fundamentals (Gribakin and Lee, 2006a). Consider a small polyatomic molecule which supports a bound positron state with a small binding energy $\varepsilon_{b}=\kappa^{2} / 2 \ll 1$. The wave function of the bound positron is very diffuse. Outside the molecule it behaves as $\varphi_{0}(\mathbf{r})=A r^{-1} e^{-\kappa r}$, with $A$ given by Eq. (34).

Suppose that the vibrational modes in this molecule are not mixed with overtones or combination vibrations. Due to the weakness of the positron binding, the vibrational excitation energies of the positron-molecule complex are close to the vibrational fundamentals $\omega_{\nu}$ of the neutral molecule, $E_{\nu} \approx \omega_{\nu}{ }^{11}$ In this case the sum in Eq. (38) is over the modes $\nu$, and the resonant energies are

\footnotetext{
${ }^{11}$ There is extensive experimental evidence that $E_{\nu} \approx \omega_{\nu}$ for most resonances observed (cf. Secs. IV and V). Apparent exceptions, where shifts $\sim 10-20 \mathrm{meV}$ are observed are the $\mathrm{C}-\mathrm{H}$ stretch mode of $\mathrm{CH}_{3} \mathrm{~F}$ and the $\mathrm{O}-\mathrm{H}$ stretch in methanol (cf. Sec. IV).
} 
$\varepsilon_{\nu}=\omega_{\nu}-\varepsilon_{b}$. Some (or all) of these modes can be IR active. Positron capture into the corresponding VFR is mediated by the long-range dipole coupling, and one can readily evaluate this contribution to $Z_{\mathrm{eff}}^{\text {(res) }}$.

Consider a positron with momentum $\mathbf{k}$ incident upon a molecule in the vibrational ground state $\Phi_{0}(\mathbf{R})$, where $\mathbf{R}$ represents all of the molecular coordinates. If $k^{2} / 2$ $\approx \varepsilon_{\nu}$, the positron can be captured into a VFR and thus be bound to the molecule in a vibrationally excited state $\Phi_{\nu}(\mathbf{R})$. The corresponding rate $\Gamma_{\nu}^{e}$, given by Eq. (22), can be found using a method similar to the Born dipole approximation (Lane, 1980) with the coupling $V=\hat{\mathbf{d}} \cdot \mathbf{r} / r^{3}$, where $\hat{\mathbf{d}}$ is the dipole moment operator of the molecule. ${ }^{12}$ This gives the amplitude

$$
\begin{aligned}
\left\langle\Phi_{\nu}|V| \Psi_{\mathbf{k}}^{(0)}\right\rangle & =\int \varphi_{0}(\mathbf{r}) \Phi_{\nu}^{*}(\mathbf{R}) \frac{\hat{\mathbf{d}} \cdot \mathbf{r}}{r^{3}} e^{i \mathbf{k} \cdot \mathbf{r}} \Phi_{0}(\mathbf{R}) d \mathbf{r} d \mathbf{R} \\
& =\frac{4 \pi i}{3} \frac{\mathbf{d}_{\nu} \cdot \mathbf{k}}{\sqrt{2 \pi \kappa}}{ }_{2} F_{1}\left(\frac{1}{2}, 1 ; \frac{5}{2} ;-\frac{k^{2}}{\kappa^{2}}\right),
\end{aligned}
$$

where $\mathbf{d}_{\nu}=\left\langle\Phi_{\nu}|\hat{\mathbf{d}}| \Phi_{0}\right\rangle$ and ${ }_{2} F_{1}$ is the hypergeometric function. ${ }^{3}$ The corresponding elastic rate is

$$
\Gamma_{\nu}^{e}=\frac{16 \omega_{\nu} d_{\nu}^{2}}{27} h(\xi)
$$

where

$$
h(\xi)=\xi^{3 / 2}(1-\xi)^{-1 / 2}\left[{ }_{2} F_{1}\left(\frac{1}{2}, 1 ; \frac{5}{2} ;-\frac{\xi}{1-\xi}\right)\right]^{2}
$$

is a dimensionless function of $\xi=1-\varepsilon_{b} / \omega_{\nu}$, with a maximum $h \approx 0.75$ at $\xi \approx 0.89$.

Equation (40) shows that the elastic rate for the resonance of an IR-active mode is determined largely by its frequency $\omega_{\nu}$ and transition dipole amplitude $d_{\nu}$. Their values are known for many species from IR absorption measurements [see, e.g., Bishop and Cheung (1982)].

This theory has been successfully applied to the methyl halides (Gribakin and Lee, 2006a). Energy-resolved measurements of $Z_{\text {eff }}$ for $\mathrm{CH}_{3} \mathrm{~F}$ (Barnes et al., 2003), $\mathrm{CH}_{3} \mathrm{Cl}$, and $\mathrm{CH}_{3} \mathrm{Br}$ (Barnes et al., 2006) show peaks close to the vibrational mode energies, pointing to a sizable contribution of resonant annihilation in these molecules. The methyl halides have $C_{3 v}$ symmetry, and all six vibrational modes are IR active (see Table II for $\mathrm{CH}_{3} \mathrm{Cl}$ ). Methyl halides are also relatively small so that IVR effects may not be important in the energy range of the fundamentals (see Sec. II.G). Thus for these molecules, Eqs. (36), (38), and (40) allow one to calculate the contribution of all VFR to $Z_{\mathrm{eff}}^{\text {(res) }}$.

The only free parameter in the theory is the positron binding energy. It can be chosen by comparison with the

\footnotetext{
${ }^{12}$ Experiments show that the Born dipole approximation provides good estimates or lower bounds, for the excitation cross sections of IR-active modes by low-energy positrons (Marler and Surko, 2005; Marler et al., 2006).

${ }^{13}$ Here ${ }_{2} F_{1}\left(\frac{1}{2}, 1 ; \frac{5}{2} ;-z^{2}\right)=\frac{3}{2} z^{-2}\left[\left(1+z^{2}\right) z^{-1} \arctan z-1\right]$.
}

TABLE II. Characteristics of the vibrational modes of $\mathrm{CH}_{3} \mathrm{Cl}$.

\begin{tabular}{lccccc}
\hline \hline Mode & Symmetry & $b_{\nu}$ & $\begin{array}{c}\omega_{\nu}{ }^{\mathrm{a}} \\
(\mathrm{meV})\end{array}$ & $\begin{array}{c}d_{\nu}{ }^{\mathrm{a}} \\
(\mathrm{a} . \mathrm{u} .)\end{array}$ & $\begin{array}{c}\Gamma_{\nu}{ }^{e \mathrm{a}} \\
(\mu \mathrm{eV})\end{array}$ \\
\hline$\nu_{1}$ & $A_{1}$ & 1 & 363 & 0.0191 & 57.2 \\
$\nu_{2}$ & $A_{1}$ & 1 & 168 & 0.0176 & 22.9 \\
$\nu_{3}$ & $A_{1}$ & 1 & 91 & 0.0442 & 65.7 \\
$\nu_{4}$ & $E$ & 2 & 373 & 0.0099 & 15.9 \\
$\nu_{5}$ & $E$ & 2 & 180 & 0.0162 & 20.9 \\
$\nu_{6}$ & $E$ & 2 & 126 & 0.0111 & 6.4 \\
\hline \hline
\end{tabular}

${ }^{a}$ Mode energies and transition amplitudes from Bishop and Cheung (1982); elastic widths from Eq. (40) for $\varepsilon_{b}=25 \mathrm{meV}$.

experimental $Z_{\text {eff }}$ spectrum. To do this, the theoretical $Z_{\text {eff }}$ must be averaged over the energy distribution of the positron beam $f_{\mathrm{b}}(\varepsilon-\bar{\varepsilon})$ from Eq. (56),

$$
\bar{Z}_{\mathrm{eff}}^{(\mathrm{res})}(\bar{\varepsilon})=\int Z_{\mathrm{eff}}^{(\mathrm{res})}(\varepsilon) f_{\mathrm{b}}(\varepsilon-\bar{\varepsilon}) d \varepsilon .
$$

This integral is simplified by the fact that the resonances are very narrow since the total width $\Gamma_{\nu}=\Gamma_{\nu}^{e}+\Gamma_{\nu}^{a}$ is small compared to the energy spread of the beam. For example, the values in Table II show that $\Gamma_{\nu}^{e}<0.1 \mathrm{meV}$. The annihilation width is even smaller; for $\varepsilon_{b}=25 \mathrm{meV}$, Eq. (35) yields $\Gamma_{\nu}^{a}=0.15 \mu \mathrm{eV}$. Hence,

$$
\bar{Z}_{\mathrm{eff}}^{(\mathrm{res})}(\bar{\varepsilon})=2 \pi^{2} \rho_{e p} \sum_{\nu} \frac{b_{\nu} \Gamma_{v}^{e}}{k_{\nu} \Gamma_{\nu}} \Delta\left(\bar{\varepsilon}-\varepsilon_{\nu}\right)
$$

where $k_{\nu}=\sqrt{2 \varepsilon_{\nu}}$ and $\Delta(E) \equiv f_{\mathrm{b}}(-E)$ describes the shape of the resonances as measured in the positron-beam experiment (see Sec. III.D).

The above estimates show that $\Gamma_{\nu}^{a} \ll \Gamma_{\nu}^{e}$. In this case the total decay rate is dominated by the elastic rate $\Gamma_{\nu} \approx \Gamma_{\nu}^{e}$, and the contributions of individual resonances to the sum in Eq. (42) are not sensitive to the precise values of $\Gamma_{\nu}^{e}$. Therefore even relatively weak positron-vibrational coupling is sufficient to fully "turn on" the resonance contribution. This explains why the $Z_{\text {eff }}$ and IR absorption spectra of a molecule can be quite different even when the resonant $Z_{\text {eff }}$ is determined by dipole coupling.

Application of this theory to methyl halides and other small molecules is discussed in Sec. IV.

\section{G. Resonant annihilation in large molecules}

\section{Vibrational level densities}

In general, a molecule with $N$ atoms has $3 N-6$ vibrational degrees of freedom. If positron attachment proceeds only via excitation of the single-mode VFRs, the resonant $Z_{\text {eff }}$ values will grow linearly with the size of the molecule. However, the experimental $Z_{\text {eff }}$ data show a much faster increase (cf. the data for alkanes in Table I). These large $Z_{\text {eff }}$ values can only be explained if the positrons can couple, at least indirectly, with multiquantum vibrations. 
In large polyatomic species, the total vibrational level density increases rapidly with the excitation energy and is quite high, even in the energy range of the fundamentals. The spacing between the multimode VFRs in such spectra is small compared with the energy spread of the incident positrons. Averaging $Z_{\mathrm{eff}}^{(\mathrm{res})}$ from Eq. (38) over an energy interval which contains many resonances near positron energy $\varepsilon$, one obtains

$$
Z_{\mathrm{eff}}(\varepsilon)=\frac{2 \pi^{2} \rho_{e p}}{k}\left\langle\frac{\Gamma^{e}(\varepsilon)}{\Gamma(\varepsilon)}\right\rangle \rho\left(\varepsilon+\varepsilon_{b}\right),
$$

where $\rho\left(\varepsilon+\varepsilon_{b}\right)$ is the vibrational level density of the positron-molecule complex. From now on we omit the superscript in $Z_{\text {eff }}^{(\text {res })}$, since $Z_{\text {eff }}$ in large molecules is almost entirely due to resonant annihilation. In Eq. (43) it is assumed that the positron collides with a molecule in the ground vibrational state. Larger molecules have a significant thermal energy content at room temperature, which can be taken into account (see below).

The $Z_{\text {eff }}$ in Eqs. (38) and (43) and the contributions of individual resonances in Eq. (42) are proportional to $\rho_{e p} / k$. Since $\rho_{e p} \propto \sqrt{\varepsilon_{b}}$ [cf. Eq. (36)], the resonant contribution to $Z_{\text {eff }}$ is proportional to the dimensionless "kinematic" factor $g=\sqrt{\varepsilon_{b} / \varepsilon}$. The magnitude and energy dependence of $Z_{\text {eff }}$ beyond this factor are due to the dynamics of molecular vibrations and positron interaction with them, etc. Normalizing $Z_{\text {eff }}$ by $g$ has proven to be useful in analyzing various trends in resonant annihilation (Young and Surko, 2007) (see Secs. IV and V).

According to Eq. (43), the resonant $Z_{\text {eff }}$ is proportional to the vibrational spectrum density $\rho$. This density can be evaluated easily in the harmonic approximation in which $E_{\nu}=\Sigma_{k} n_{k} \omega_{k}$, where $n_{k}$ are non-negative integers and $\omega_{k}$ are the mode frequencies. For weakly bound positron-molecule complexes, these frequencies are close to those of the neutral molecule and can be taken from experiment (Linstrom and Mallard, 2005) or quantum chemistry calculations [see, e.g., using Q-Chem, Kong et al. (2000)]. To apply Eq. (43) to the annihilation of thermal positrons at temperature $T$, the density must be averaged over the Maxwellian positron energy distribution,

$$
\bar{\rho}\left(\varepsilon_{b}\right)=\int_{0}^{\infty} \rho\left(\varepsilon_{b}+k^{2} / 2\right) \frac{e^{-k^{2} / 2 k_{B} T}}{\left(2 \pi k_{B} T\right)^{3 / 2}} 4 \pi k^{2} d k,
$$

where $k_{B}$ is the Boltzmann constant. Figure 4 shows $\bar{\rho}\left(\varepsilon_{b}\right)$ for alkanes with $n=3-9$ carbons. These densities increase rapidly with the positron binding energy and with the size of the alkane molecule.

The estimates made in Sec. II.F show that $\Gamma^{e} / \Gamma \approx 1$ for the VFRs of IR-active modes. If the same were true for the multimode resonances, the increase in $Z_{\text {eff }}$ values along the alkane series would match the growth of their vibrational level densities as per Eq. (43). However, experimental room-temperature $Z_{\text {eff }}$ values increase at a much slower rate. As a result, the effective vibrational densities $\rho$ estimated using Eqs. (43) and (36) from experimental thermal $Z_{\text {eff }}$ values and binding energies

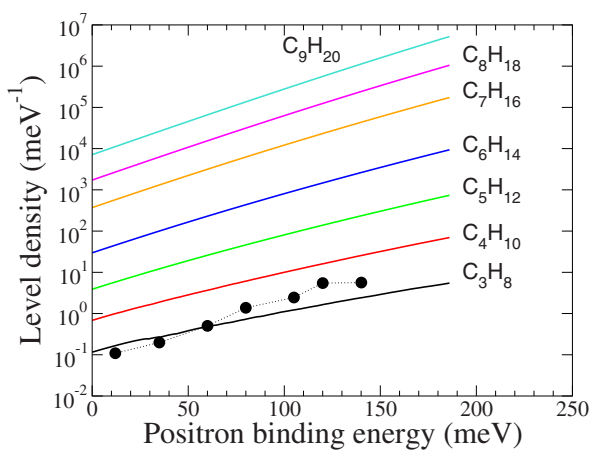

FIG. 4. (Color online) Vibrational level densities in alkanes as functions of the positron binding energy: solid curves, calculated from Eq. (44) for room-temperature incident positrons; circles, estimated from experimental $Z_{\text {eff }}$ values at $300 \mathrm{~K}$ for $\mathrm{C}_{3} \mathrm{H}_{8}$ to $\mathrm{C}_{9} \mathrm{H}_{20}$, using Eqs. (36) and (43) and assuming $\Gamma^{e} / \Gamma$ $=1$, and plotted against the experimental binding energies (Table V). From Gribakin and Gill, 2004.

(Table $\mathrm{V}$ ) and assuming $\Gamma^{e} / \Gamma=1$, are much lower than the calculated densities for all alkanes larger than propane (see Fig. 4). Hence the above assumption is generally incorrect and, instead, $\Gamma^{e} / \Gamma \ll 1$ (Gribakin and Gill, 2004).

What is the physical reason for the suppressed $\Gamma^{e} / \Gamma$ ratio? The positron coupling to multimode VFR is likely much weaker than that of single-mode resonances. For example, the dipole coupling analyzed in Sec. II.F can cause only single-quantum vibrational transitions (in the harmonic approximation). Combination vibrations and overtones can be excited due to anharmonic or Coriolis terms in the vibrational Hamiltonian. In this case, the coupling strength of a single-mode excitation is divided between many multiquantum excitations. The value of $\Gamma^{e}$ for a multimode VFR is then only a small fraction of the typical single-mode $\Gamma^{e}$. This is discussed in more detail in Sec. II.G.2. In addition, some vibrational excitations may be completely inaccessible to the incident positron (e.g., due to symmetry). This will reduce the average $\left\langle\Gamma^{e} / \Gamma\right\rangle$ or, effectively, reduce the vibrational density in Eq. (43).

Another reason for the reduction of $\Gamma^{e} / \Gamma$ could be the contribution of vibrationally inelastic escape to the total width $\Gamma$. At present there is little direct experimental evidence of inelastic positron escape (see Sec. V). However, if the system was in the regime of strong mixing between the single-mode and multimode vibrational excitations, then one cannot see why such channels would be closed. ${ }^{14}$

A simple estimate of this effect can be made assuming complete statistical mixing of all vibrational excitations

\footnotetext{
${ }^{14}$ Vibrationally inelastic scattering following capture has been studied in electron-molecule collisions via energy-loss spectra. In addition to the excitation of modes and overtones, vibrational state "quasicontinua" have been observed in larger polyatomic molecules at electron-volt excitation energies (Allan, 1984).
} 


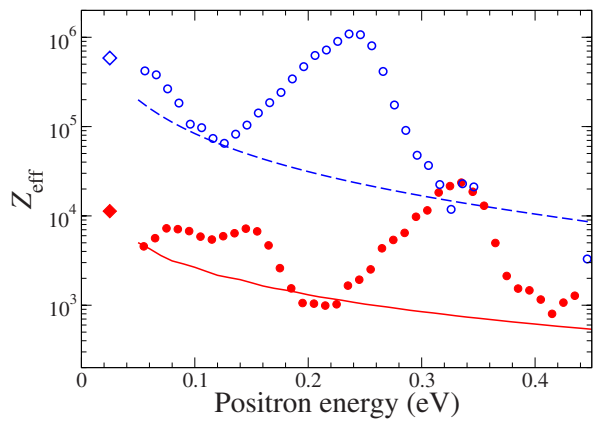

FIG. 5. (Color online) Comparison of experimental $Z_{\text {eff }}$ for butane (solid symbols) and octane (open symbols) with the predictions of the statistical model: diamonds, experimental $Z_{\text {eff }}^{\text {th }}$ values for thermal positrons at $300 \mathrm{~K}$ (cf. Table V); circles, $Z_{\text {eff }}$ measured as a function of positron energy (Barnes et al., 2003); curves, $Z_{\text {eff }}$ calculated from Eq. (45), using $\varepsilon_{b}$ fitted to reproduce thermal $Z_{\text {eff }}$.

(Gribakin and Lee, 2009). When a positron with energy $\varepsilon$ collides with a molecule with vibrational energy $E_{v}$ then all final vibrational states with energies $E_{v}^{\prime}<E_{v}+\varepsilon$ can be populated. Assuming that the positron coupling strengths to all vibrational excitations are similar, one has $\Gamma^{e} / \Gamma \approx 1 / N\left(\varepsilon+E_{v}\right)$, where $N\left(\varepsilon+E_{v}\right)=\int_{0}^{\varepsilon+E_{v}} \rho\left(E_{v}^{\prime}\right) d E_{v}^{\prime}$ is the number of open vibrational escape channels. Equation (43) then becomes

$$
Z_{\mathrm{eff}} \approx \frac{2 \pi^{2} \rho_{e p}}{k} \frac{\rho\left(\varepsilon+E_{v}+\varepsilon_{b}\right)}{N\left(\varepsilon+E_{v}\right)}
$$

For a given molecule this expression contains only one free parameter, namely, the positron binding energy.

The binding energies can be chosen by comparison with experimental room-temperature data for thermal positrons (Table V), averaging $Z_{\text {eff }}$ from Eq. (45) over the initial target states using the Boltzmann factors $\exp \left(-E_{v} / k_{B} T\right)$, and the Maxwellian positron energy distribution. For alkanes with 3-8 carbons, such a fit gives the binding energies $\varepsilon_{b}=22,42,65,90,103$, and $122 \mathrm{meV}$, respectively. These values are in good agreement with those measured in the positron-beam experiments (cf. Table V). However, the dependence of $Z_{\text {eff }}$ on the positron energy predicted by Eq. (45) is in striking disagreement with the measured energy-resolved $Z_{\text {eff }}$, as shown in Fig. 5 for butane and octane. The $Z_{\text {eff }}$ values from Eq. (45) decrease monotonically, the increase in $N\left(\varepsilon+E_{v}\right)$ being faster than that of the density $\rho$. In contrast, the experimental $Z_{\text {eff }}$ spectra show resonant peaks corresponding to the vibrational fundamentals (downshifted by $\varepsilon_{b}$, cf. Fig. 1), and the peak $Z_{\text {eff }}$ values exceed the predictions of Eq. (45) by a factor of $\sim 50$. Thus the model that assumes complete statistical mixing with all vibrational excitations coupled to the positron continuum does not explain either the resonant structure or the large values of $Z_{\text {eff }}$ that are observed.

\section{Mode-based resonant doorway states}

The energy dependence of the measured $Z_{\text {eff }}$ in alkanes shown in Fig. 5 is of the type expected for modebased VFRs described in Sec. II.F. On the other hand, the magnitude of $Z_{\text {eff }}$ and its increase with the size of the molecule are much greater than the contribution of mode-based resonances. This suggests a two-step model of positron capture that involves mode-based vibrational doorway resonances ${ }^{15}$ (Gribakin and Gill, 2004).

In this model, the incident positron first forms a quasibound state with the molecule by transferring its excess energy to a single mode with near-resonant energy $\omega_{n} \approx \varepsilon+\varepsilon_{b}$. This simple doorway state of the positronmolecule complex [also termed a zeroth-order "bright" state (Nesbitt and Field, 1996)] is embedded in the dense spectrum of multimode vibrations (or "dark" states, as they are not coupled directly to the positron continuum). Due to vibrational state mixing caused by anharmonic or rotationally induced coupling terms in the vibrational Hamiltonian, the doorway state can then "spread" into multimode vibrational states. This process of vibrational energy redistribution takes place on a time scale $\tau$ $\sim 1 / \Gamma_{\mathrm{spr}}$, where $\Gamma_{\mathrm{spr}}$ is known as the spreading width in nuclear physics, or the IVR rate in molecular physics.

To link the multimode-VFR and the doorway-stateresonance pictures, consider a perturbative expression for the elastic rate,

$$
\Gamma_{\nu}^{e}=2 \pi\left|\left\langle\Psi_{\nu}|V| 0, \varepsilon\right\rangle\right|^{2},
$$

where $|0, \varepsilon\rangle$ describes the positron incident on the ground-state molecule. ${ }^{16}$ The multimode eigenstate of the positron-molecule complex $\left|\Psi_{\nu}\right\rangle$ can be expanded in the basis of the noninteracting (e.g., harmonic approximation) multimode vibrational states $\left|\Phi_{i}\right\rangle$,

$$
\left|\Psi_{\nu}\right\rangle=\sum_{i} C_{i}^{(\nu)}\left|\Phi_{i}\right\rangle
$$

where the coefficients $C_{i}^{(\nu)}$ are obtained by diagonalizing the vibrational Hamiltonian in the basis of $\left|\Phi_{i}\right\rangle$.

Assume that of all $\left|\Phi_{i}\right\rangle$, only those which describe a bound positron and a single-mode excitation (i.e., $\left|n, \varepsilon_{0}\right\rangle$, where $n$ indicates the mode) are coupled to $|0, \varepsilon\rangle$. These states $\left|n, \varepsilon_{0}\right\rangle$ are the doorway states introduced above. The coefficients $C_{i}^{(\nu)}$ describe the mixing of the doorway states with the multimode eigenstates $\nu$ (i.e., "spreading"). The corresponding probabilities can be approximated by a Breit-Wigner line shape,

\footnotetext{
${ }^{15}$ The term "doorway resonance" originates in nuclear physics, where it means "a metastable state formed in the initial state of the reaction," which "may decay partly into the open channels (direct reactions), and partly through the coupling to the internal degrees of freedom" (Bohr and Mottelson, 1998).

${ }^{16}$ See Eq. (22); for simplicity, in Eq. (46) we assume that only one positron partial wave contributes to the rate and that its wave function is normalized to a $\delta$ function in energy rather than to a plane wave.
} 


$$
\left|C_{i}^{(\nu)}\right|^{2} \propto \frac{\Gamma_{\mathrm{spr}}^{2} / 4}{\left(E_{\nu}-E_{i}\right)^{2}+\Gamma_{\mathrm{spr}}^{2} / 4},
$$

subject to normalization $\Sigma_{i}\left|C_{i}^{(\nu)}\right|^{2}=1$. Here $E_{\nu}$ and $E_{i}$ are the energies of the eigenstate and basis state of the positron-molecule complex, respectively, so that $E_{\nu} \approx \varepsilon$ $-\varepsilon_{0}=\varepsilon+\varepsilon_{b}$ and $E_{i}=\omega_{n}$ for $\left|\Phi_{i}\right\rangle=\left|n, \varepsilon_{0}\right\rangle$.

Using Eqs. (43) and (46)-(48), one obtains $Z_{\text {eff }}$, averaged over energy on the scale of closely spaced VFRs, as

$$
Z_{\mathrm{eff}}=\frac{2 \pi^{2} \rho_{e p}}{k} \frac{\Gamma_{\mathrm{spr}}}{2 \pi \Gamma(\varepsilon)} \sum_{n} \frac{\Gamma_{n}^{e}}{\left(\varepsilon-\omega_{n}+\varepsilon_{b}\right)^{2}+\frac{1}{4} \Gamma_{\mathrm{spr}}^{2}},
$$

where $\Gamma_{n}^{e}=2 \pi\left|\left\langle n, \varepsilon_{0}|V| 0, \varepsilon\right\rangle\right|^{2}$ is the elastic rate of the doorway of mode $n$. Equation (49) has the same form as Eq. (38), except that it contains the elastic rates of the doorways, $\Gamma_{n}^{e}$, and the sum is over the modes.

Comparison with Eq. (43) shows that the resonant energy dependence of $Z_{\text {eff }}$ in Eq. (49) is due to the modulation of the elastic rate by the mode-based doorways. Evaluating $Z_{\text {eff }}$ from Eq. (49) at the doorway resonance energy (i.e., $\varepsilon=\omega_{n}-\varepsilon_{b}$ ) and comparing it with Eq. (43) gives an estimate of the elastic rate of the VFR:

$$
\Gamma^{e}(\varepsilon) \sim \frac{2 \Gamma_{n}^{e}}{\pi \rho\left(\varepsilon+\varepsilon_{b}\right) \Gamma_{\mathrm{spr}}} .
$$

The product $\rho\left(\varepsilon+\varepsilon_{b}\right) \Gamma_{\mathrm{spr}}$ is the number of vibrational eigenstates within the energy interval $\Gamma_{\mathrm{spr}}$. In the regime of strong level mixing this number is large, $\rho\left(\varepsilon+\varepsilon_{b}\right) \Gamma_{\mathrm{spr}}$ $\gg 1$. Equation (50) shows that the elastic rates of the multimode VFRs are much smaller than those of the doorways, as each VFR carries a small fraction of the positron coupling strength of the doorway.

Equation (49) shows that the enhancement of $Z_{\text {eff }}$ for larger molecules is caused by (i) larger binding energies and $\rho_{e p} \propto \sqrt{\varepsilon_{b}}$, (ii) larger number of modes to doorways in the sum, and most importantly (iii) smaller total decay rates $\Gamma(\varepsilon)$ (i.e., longer lifetimes) of the VFRs. This decrease in $\Gamma(\varepsilon)$ must be related to the suppression of $\Gamma^{e}$ due to higher level densities. The total rate is, however, always bounded from below by the annihilation contribution $\Gamma(\varepsilon)>\Gamma^{a}$. The annihilation rates are typically smaller than the elastic rates of mode-based doorways by two to three orders of magnitude (see Sec. II.F). Hence, one can expect a similar-sized increase in the contribution to $Z_{\text {eff }}$ from each mode-based doorway resonance in a large molecule, compared with that in a small molecule.

Figure 6 shows the applicability of Eq. (49). Here it is used to fit the experimental energy-resolved $Z_{\text {eff }}$ data for butane and octane (Barnes et al., 2003). For this comparison, $Z_{\text {eff }}$ from Eq. (49) is averaged over the positron energy distribution (Sec. III.D). The result does not depend on the spreading width as long as it is much smaller than the typical energy spread of the beam (i.e., $\Gamma_{\mathrm{spr}}$ $\ll 40 \mathrm{meV}$ ). The binding energies of the two species are chosen to be $\varepsilon_{b}=35$ and $122 \mathrm{meV}$. The remaining unknown parameter is the ratio $\Gamma_{n}^{e} / \Gamma(\varepsilon)$. Given the differences between the $\mathrm{C}-\mathrm{H}$ stretch and lower-energy reso-

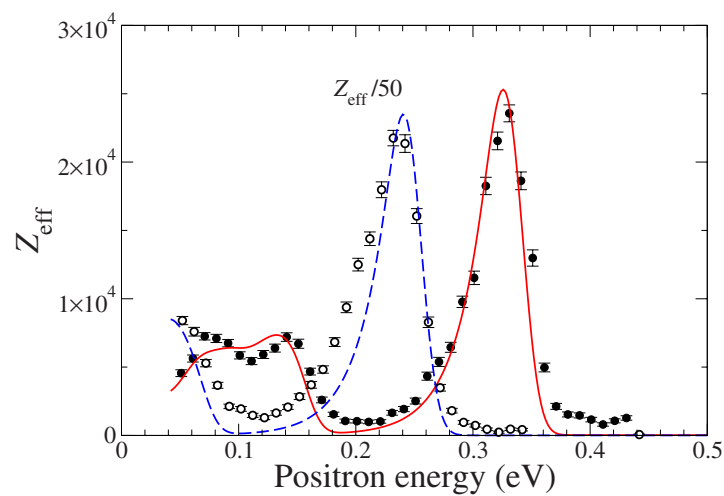

FIG. 6. (Color online) Comparison of the experimental $Z_{\text {eff }}$ for (-) butane, $\mathrm{C}_{4} \mathrm{H}_{10}$ and $(\bigcirc)$ octane, $\mathrm{C}_{8} \mathrm{H}_{18}$ (scaled by a factor 1/50) (Barnes et al., 2003), with $Z_{\text {eff }}$ from Eq. (49). The theoretical $Z_{\text {eff }}$ is averaged over the positron beam energy distribution. The fit for butane uses $\varepsilon_{b}=35 \mathrm{meV}, \Gamma_{n}^{e} / \Gamma=7.2$ for $\mathrm{C}-\mathrm{H}$ stretch modes and $\Gamma_{n}^{e} / \Gamma=1.2$ for the rest; for octane, $\varepsilon_{b}$ $=122 \mathrm{meV}, \Gamma_{n}^{e} / \Gamma=84$ for $\mathrm{C}-\mathrm{H}$ stretch modes and $\Gamma_{n}^{e} / \Gamma=14$ for the rest. From Gribakin and Lee, 2009.

nances in Fig. 6, two different values are used for these groups of modes, the former six times greater than the latter. The ratios $\Gamma_{n}^{e} / \Gamma$ for octane are approximately 12 times larger than for butane. In the context of the model, this reflects the greater degree of mixing between multimode VFRs in the larger molecule.

The results shown in Fig. 6 summarize the extent of our understanding of annihilation in large molecules. To explain the observed magnitudes of $Z_{\text {eff }}$, positron capture into multimode VFR must be invoked, mediated by a process such as IVR. However, if the enhancement is indeed due to IVR, it appears to be far from statistically complete.

\section{Annihilation and the onset of IVR}

Experimentally, resonant annihilation is observed in relatively small polyatomics with four or five atoms (Sec. IV), as well as in much larger molecules (Sec. V). In the first case, the annihilation is due to the single-mode VFRs, possibly augmented by contributions of combination and overtone resonances (see Sec. IV.B). In large molecules enhanced $Z_{\text {eff }}$ values are assumed to be due to the spreading of the vibrational energy into multimode VFRs. The energy dependence of $Z_{\text {eff }}$ in large molecules is typically well represented by the spectrum of the fundamentals, which act as doorways. Isolated overtone and combination VFRs cannot, in general, be identified as distinct doorways in the $Z_{\text {eff }}$ spectrum, though there are exceptions (see, e.g., benzene, Sec. V). This can probably be explained by the much smaller ratios of $\Gamma_{n}^{e} / \Gamma(\varepsilon)$ for the combination or overtone doorways [cf. Eq. (49)].

The transition from the small- to the large-molecule behavior occurs with the onset of strong vibrational mixing (i.e., IVR) involving, in particular, the vibrational fundamentals. The phenomenon of IVR has been studied widely using a number of techniques (Nesbitt and Field, 1996). In particular, high-resolution $\left(0.0005 \mathrm{~cm}^{-1}\right)$ 
TABLE III. Vibrational excitation densities for zero- and room-temperature molecules at the $\mathrm{C}-\mathrm{H}$ stretch mode energy.

\begin{tabular}{lcccc}
\hline \hline Molecule & & $\begin{array}{c}\rho^{\mathrm{a}} \\
\left(1 / \mathrm{cm}^{-1}\right)\end{array}$ & $\begin{array}{c}\rho_{T}{ }^{\mathrm{a}} \\
\left(1 / \mathrm{cm}^{-1}\right)\end{array}$ & $\begin{array}{c}\text { Dilution } \\
\text { factors }\end{array}$ \\
\hline Acetylene & $\mathrm{C}_{2} \mathrm{H}_{2}$ & 0.05 & 0.06 & \\
Methyl chloride & $\mathrm{CH}_{3} \mathrm{Cl}$ & 0.07 & 0.07 & \\
Ethylene & $\mathrm{C}_{2} \mathrm{H}_{4}$ & 0.08 & 0.09 & \\
Methanol & $\mathrm{CH}_{3} \mathrm{OH}$ & 0.12 & 0.13 & \\
Ethane & $\mathrm{C}_{2} \mathrm{H}_{6}$ & 0.42 & 0.54 & $0.8,1$ \\
Cyclopropane & $\mathrm{C}_{3} \mathrm{H}_{6}$ & 0.59 & 0.81 & $0.3,0.7,0.8$ \\
Ethanol & $\mathrm{C}_{2} \mathrm{H}_{5} \mathrm{OH}$ & 2.84 & 6.22 & \\
Propane & $\mathrm{C}_{3} \mathrm{H}_{8}$ & 5.19 & 14.3 & $0.2,0.4$ \\
Benzene & $\mathrm{C}_{6} \mathrm{H}_{6}$ & 5.76 & 22.3 & $0.6,0.7,0.9$ \\
Butane & $\mathrm{C}_{4} \mathrm{H}_{10}$ & 106 & 921 & $0.16,0.05^{\mathrm{c}}$ \\
\hline \hline
\end{tabular}

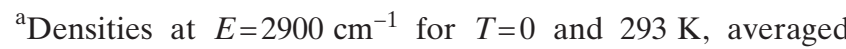
over a Gaussian with $10 \mathrm{meV}$, FWHM. Mode frequencies for most molecules are from Linstrom and Mallard (2005).

${ }^{b}$ Values for the C-H stretch modes (Stewart and McDonald, 1983).

${ }^{c}$ Values for 1-butyne (Kim et al., 1987) and isobutane (Stewart and McDonald, 1983), respectively.

measurements of vibrational spectra of jet-cooled molecules allow direct observation of the splitting of singlemode transitions into clumps of vibrationally mixed multimode levels spread over an energy interval $\Gamma_{\mathrm{spr}}$ $\sim 0.02 \mathrm{~cm}^{-1} \approx 2 \mu \mathrm{eV}$ (McIlroy and Nesbitt, 1990).

Another technique used to study IVR in the range of the C-H stretch modes is IR fluorescence (Stewart and McDonald, 1983). Here a fundamental vibration is excited by a short laser pulse. If this vibration is mixed with multimode excitations, the amount of fluorescence at the fundamental frequency is reduced. The measured fluorescence, normalized to the known IR absorption strength, gives the "dilution factor." Its reciprocal characterizes the number of eigenstates that are strongly coupled to the fundamental. If all vibrational states within the energy range $\Gamma_{\mathrm{spr}}$ are mixed, the dilution factor will be $\sim\left(\rho \Gamma_{\mathrm{spr}}\right)^{-1}$, where $\rho$ is the vibrational spectrum density at the relevant energy. Note that a similar factor enters Eq. (50). Stewart and McDonald (1983) observed that the dilution factor drops rapidly when the level density is increased beyond the "threshold" value of $\rho=10-100 / \mathrm{cm}^{-1}$. This is in agreement with the value $\Gamma_{\mathrm{spr}} \sim 0.02 \mathrm{~cm}^{-1}$, as $\rho \Gamma_{\mathrm{spr}} \geqslant 1$ marks the onset of IVR.

In order to see if a similar threshold governs the transition from the small- to the large-molecule behavior in annihilation, vibrational densities have been evaluated for a number of molecules using the harmonic approximation. The densities at the excitation energy of the C-H stretch mode $\left(E=2900 \mathrm{~cm}^{-1}=0.36 \mathrm{eV}\right)$ are shown in Table III. To assess the effect of finite molecular temperature, the densities were evaluated both at zero temperature, denoted $\rho(E)$, and at room temperature, $\rho_{T}(E)=\Sigma_{v} \rho\left(E+E_{v}\right) e^{-E_{v} / k_{B} T} / \Sigma_{v} e^{-E_{v} / k_{B} T}$. In Table III val- ues of the IR fluorescence dilution factor, where known, are also listed.

Energy-resolved $Z_{\text {eff }}$ measurements put the top four molecules in Table III in the small-molecule category (cf. Sec. IV). Propane, benzene, and butane at the bottom of the table behave as large molecules (Sec. V), as they exhibit $Z_{\text {eff }}$ values that cannot be explained by the mode-based VFR. Ethane and cyclopropane appear to be borderline, with characteristics that place them in both categories (e.g., the C-H stretch peak in both molecules appears to be strongly enhanced). The values of the densities in Table III are broadly in agreement with this classification, with the threshold density, $\rho \sim 1 / \mathrm{cm}^{-1}$. This value is lower than the threshold density in the fluorescence studies, perhaps due to differences in the nature of the vibrational energy transfer in the two cases. Somewhat surprisingly, $Z_{\text {eff }}$ measurements for ethanol (cf. Sec. IV.D) indicate that it is a "small molecule," in contrast with its vibrational density value. This suggests that the threshold value depends on the details of the vibrational Hamiltonian.

Table III shows that molecular temperature can strongly affect the density for larger molecules. The only experiments to investigate the effect of molecular temperature on $Z_{\text {eff }}$ in similar-sized molecules, heptane and pentane, show only a small effect at the $\mathrm{C}-\mathrm{H}$ stretch peak (Sec. V.E.2). While the analysis here indicates that molecules with $\rho \sim 1 / \mathrm{cm}^{-1}$ might change from exhibiting small- to large-molecule behavior as the temperature is increased, molecules with these density values exhibit only small changes in $\rho$ with temperature. Thus the combination of this analysis and the experimental results on large molecules indicate that temperature likely plays a limited role in changing the details of the $Z_{\text {eff }}$ spectra.

When the level densities for larger molecules are examined as a function of the excitation energy $E$, the densities in the $\mathrm{C}-\mathrm{H}$ stretch energy range are an order of magnitude greater than those in the range of other fundamentals, $E \leqq 1000 \mathrm{~cm}^{-1}$. In principle, this could explain the stronger enhancement of $Z_{\text {eff }}$ in the C-H stretch peak, as compared to that in the low-energy mode peaks (see Fig. 6 and Sec. V for further discussion).

To summarize, optics-based IVR studies show that the degree of IVR increases rapidly as a function of molecular size. The onset of strong IVR occurs for molecules that are similar in size to those for which mode-based VFR-mediated annihilation begins to fail to explain the $Z_{\text {eff }}$ spectra (e.g., ethane, cyclopropane, and propane). Thus, while indirect, these results fit well with the physical picture that IVR is responsible for the very large annihilation rates observed in large molecules.

\section{H. Calculations of annihilation and binding}

\section{Annihilation}

Most calculations of $Z_{\text {eff }}$ for molecules have been done for diatomics or small polyatomics with fixed nuclei, ignoring the vibrational dynamics. This is a good 
approximation for molecules that do not possess VFRs, where direct annihilation is the dominant mechanism. Such a calculation is still far from trivial, given the large role of electron-positron correlations.

The $\mathrm{H}_{2}$ molecule is an example. In this case one can construct a sufficiently flexible trial wave function for the positron and two electrons and use the generalized Kohn method to solve the scattering problem (Armour, 1984) and calculate $Z_{\text {eff }}$ (Armour and Baker, 1985). This calculation provides an accurate description of the positron elastic scattering cross section below $5 \mathrm{eV}$ (Armour et al., 1990). Calculation of $Z_{\text {eff }}$ requires the inclusion of terms with explicit dependence on the electron-positron distance (Armour and Baker, 1986). However, it still yields a thermal room-temperature annihilation rate of $Z_{\text {eff }}=10.2$, well below the experimental value of $14.8 \pm 0.2$ (McNutt et al., 1979). This discrepancy has been finally resolved using the stochastic variational method (Zhang et al., 2009), which is one of the most powerful methods for studying few-body systems.

Another $a b$ initio method used to calculate positronmolecule scattering and annihilation is the Schwinger multichannel (SMC) method (Germano and Lima, 1993; da Silva et al., 1994). In this scheme the $(Z+1)$-particle wave function is expanded in a Cartesian Gaussian basis set, with the functions centered on the atomic nuclei and on additional centers outside the molecule. The latter are important for representing electron-positron correlation effects. This method has been applied to $\mathrm{H}_{2}$ (Lino et al., 1998), $\mathrm{N}_{2}$ (de Carvalho et al., 2000), $\mathrm{C}_{2} \mathrm{H}_{4}$ (da Silva et al., 1996), and $\mathrm{C}_{2} \mathrm{H}_{2}$ (de Carvalho et al., 2003). In all cases the differential and total elastic scattering cross sections are in good agreement with the experimental data. In contrast, the calculated $Z_{\text {eff }}$ values for roomtemperature positron energies (Varella et al., 2002) are well below the measured thermal data, namely, $Z_{\text {eff }}$ $=7.3$ versus 14.8 for $\mathrm{H}_{2}$ (McNutt et al., 1979), 9.3 versus 30.5 for $\mathrm{N}_{2}$ (Heyland et al., 1982), 73 versus 1200 for $\mathrm{C}_{2} \mathrm{H}_{4}$ (Iwata et al., 1994), and 145 versus 3160 for $\mathrm{C}_{2} \mathrm{H}_{2}$ (Iwata, Greaves, and Surko, 1997). ${ }^{17}$ The discrepancy for $\mathrm{H}_{2}$ and $\mathrm{N}_{2}$ is most likely due to a lack of basis functions that describe short-range electron-positron correlations. The much larger gap between the theory and experiment for ethylene and acetylene has a different origin. As discussed in Sec. IV.C, the energy-resolved $Z_{\text {eff }}$ data for both molecules show large contributions of resonant annihilation that cannot be described by a fixed-nuclei calculation.

In spite of the failure to reproduce the $Z_{\text {eff }}$ values, the SMC calculations provide an important clue about the physics of the positron interaction with ethylene and acetylene. They show that electron-positron correlations in these systems lead to strong positron-molecule attraction that produces virtual levels close to zero energy (da

\footnotetext{
${ }^{17}$ The SMC computer code used to calculate the $Z_{\text {eff }}$ values prior to 2001 contained an extra factor of $Z$ due to a programming mistake (Varella et al., 2002), which gave an illusion of agreement with experiment.
}

Silva et al., 1996; de Carvalho et al., 2003). As a result, the calculated $Z_{\text {eff }}$ display a characteristic growth at low energies (cf. Fig. 2). Observation of resonant annihilation for these molecules (Sec. IV.C) indicates that they do, in fact, support positron bound states with $\varepsilon_{b}$ $\lesssim 10 \mathrm{meV}$. This means that a relatively small (though, computationally challenging) improvement in the SMC calculations could prove the existence of these bound states. This would represent an important step toward $a b$ initio theoretical description of resonant annihilation.

Positron interaction with acetylene (and methane) was also examined by Nishimura and Gianturco (2003) using a body-fixed vibrational close-coupling (VCC) method. In this approach, the positron-molecule interaction is modeled using a correlation-polarization potential (CPP). These calculations revealed the existence of a positron virtual state for methane $(\kappa \approx-0.15$ a.u. $)$ and acetylene $(\kappa \approx-0.01$ a.u. $)$. As in the case of the SMC calculations, it appears that only a small increase in the potential is required to turn the virtual state into the observed bound state in acetylene. Such an increase may well be within the uncertainty of the CPP method. In fact, a fixed-nuclei calculation with the existing CPP showed that the change from a virtual state to the bound states can be achieved by stretching the $\mathrm{C}-\mathrm{H}$ bonds in $\mathrm{C}_{2} \mathrm{H}_{2}, \mathrm{C}_{2} \mathrm{H}_{4}$, and $\mathrm{C}_{2} \mathrm{H}_{6}$ by 15-30\% (Nishimura and Gianturco, 2005b). In acetylene this change can also be induced by symmetric bending by about $16^{\circ}$ (Nishimura and Gianturco, 2004). However, this mechanism of bound-state formation appears to be problematic since the energy required to distort the molecule far exceeds the positron binding energy and the thermal energy of molecules at $\sim 300 \mathrm{~K}$.

The earliest calculation of positron annihilation on polyatomic molecules is probably that of Jain and Thompson (1983) for $\mathrm{CH}_{4}$ and $\mathrm{NH}_{3}$. They described the interaction between the positron and the target by means of a potential $V_{s}(\mathbf{r})+V_{p}(\mathbf{r})$, where $V_{s}(\mathbf{r})$ is the full electrostatic potential of the ground-state molecule and $V_{p}(\mathbf{r})$ is a CPP. This potential has the correct form $V_{p}(\mathbf{r}) \simeq-\alpha_{d} / 2 r^{4}$ at large distances. Solving the Schrödinger equation for the positron wave function $\varphi_{\mathbf{k}}(\mathbf{r})$ yields the scattering amplitude and cross section, and $\varphi_{\mathbf{k}}(\mathbf{r})$ is then used in Eq. (20) to calculate $Z_{\text {eff. Jain }}$ and Thompson (1983) showed that it is important to go beyond this approximation and include the effect of distortion of the electron density by the positron. This led to an increase in $Z_{\text {eff }}$ values for $\mathrm{CH}_{4}$ by a factor of 2 . Their final result for $\mathrm{CH}_{4}, Z_{\text {eff }}=99.5$ at $\varepsilon=0.025 \mathrm{eV}$, is reasonably close to, but still smaller than the experimental room-temperature value $Z_{\text {eff }}=142 \pm 1$ (Wright et al., 1983). They concluded that the remaining discrepancy could be removed in a better positron-molecule calculation. More significantly, they also concluded that the somewhat large, measured $Z_{\text {eff }}$ value was not the result of the formation of a positron-molecule complex. As discussed in Sec. II.D, this is in complete agreement with the current understanding of the way in which positron- 
molecule virtual states can enhance low-energy $Z_{\text {eff }}$ values.

An approach similar to that of Jain and Thompson (1983) has now been tested for a variety of organic and inorganic polyatomic molecules (Gianturco et al., 2001; Occhigrossi and Gianturco, 2003), with ethane and benzene being the largest. The short-range part of the CPP employed in these calculations is based on densityfunctional treatments of the electron-positron correlations, while the long-range behavior is described analytically (e.g., as $-\alpha_{d} / 2 r^{4}$, for the dipole polarization term) (Jain and Gianturco, 1991). The positron wave function from these calculations is used to calculate $Z_{\text {eff }}$ from Eq. (20). More recent calculations (Franz and Gianturco, 2006 ${ }^{18}$ employed an enhancement factor in the integrand of Eq. (8). It depends on the electron density and describes its local increase at the positron (Arponen, 1978; Boronski and Nieminen, 1986).

The main result of these fixed-nuclei calculations is that they fail to reproduce large experimental $Z_{\text {eff }}$ values $\left(>10^{2}\right.$ ) for molecules such as $\mathrm{C}_{2} \mathrm{H}_{2}, \mathrm{C}_{2} \mathrm{H}_{4}, \mathrm{C}_{2} \mathrm{H}_{6}$, and $\mathrm{C}_{6} \mathrm{H}_{6}$ (Occhigrossi and Gianturco, 2003). This is an indirect confirmation of the role of resonant annihilation involving nuclear vibrations in these molecules (see Secs. IV.C, V.C, and V.F). For smaller polyatomics, such as $\mathrm{H}_{2} \mathrm{O}$ and $\mathrm{CH}_{4}$, the computed room-temperature $Z_{\text {eff }}$ values, 167 and 65 , respectively, are a factor of 2 or 3 smaller than the experimental values (Gianturco et al., 2001). Here an adjustment in the CPP and/or the use of the enhancement factor in the calculation of $Z_{\text {eff }}$ could bring theory and experiment into agreement. Indeed, according to the energy-resolved $Z_{\text {eff }}$ measurements, neither of these molecules shows clearly discernible contributions of resonant annihilation (Sec. IV.E). Similarly, for diatomics, such as $\mathrm{H}_{2}, \mathrm{O}_{2}, \mathrm{~N}_{2}, \mathrm{NO}$, and $\mathrm{CO}$, the calculation with enhancement factors gives $Z_{\text {eff }}$ values between 10 and 40 (Franz and Gianturco, 2006), which are within a factor of 2 of experimental values for roomtemperature positrons.

There are few annihilation calculations that include the dynamic interaction between the positron and molecular vibrations. The VCC calculations of Gianturco and Mukherjee (1999) for $\mathrm{CO}_{2}$ and Gianturco and Mukherjee (2000) for $\mathrm{O}_{2}, \mathrm{~N}_{2}, \mathrm{NO}$, and $\mathrm{CO}$, showed that vibrational coupling has a relatively small effect on the annihilation rates. This is to be expected since small molecules such as these most likely do not bind the positron (cf. Sec. VI), and so the VFR mechanism is "switched off" for them. The true magnitude of the effect of vibrational coupling for these molecules remains somewhat uncertain, as the reported theoretical $Z_{\text {eff }}$ values probably suffer from an uncertainty related to the normalization error (see above). It is also surprising that the $Z_{\text {eff }}$ values obtained in these calculations, using a static potential, change little upon inclusion of the CPP

\footnotetext{
${ }^{18}$ This work also corrected a normalization error that overestimated $Z_{\text {eff }}$ values for diatomics reported earlier (Gianturco and Mukherjee, 2000).
}

since practically all other calculations show that correlations have a large effect on positron scattering and annihilation.

The zero-range-potential (ZRP) model calculations for $\mathrm{Kr}_{2}$ (a weakly bound van der Waals dimer) demonstrated a number of key features of resonant annihilation (Gribakin, 2002; Gribakin and Lee, 2006b). First, they showed that, while the potential representing each of the $\mathrm{Kr}$ atoms has no bound states, the dimer is capable of binding a positron. Second, they showed that positron binding has a relatively small effect on the vibrational frequency of the complex. It changes by only about $10 \%$ compared to the frequency of $\mathrm{Kr}_{2}$, in spite of the fact that the binding energy is relatively large (i.e., two times the vibrational quantum). Finally, this model showed the emergence of VFR in a dynamic positronmolecule calculation. The resonant contribution leads to a large increase in $Z_{\text {eff }}$ (e.g., for thermal positrons at $300 \mathrm{~K}$, from $Z_{\text {eff }}=250$ for direct annihilation to $Z_{\text {eff }}$ $=700-950$, depending on the details of the model). Unfortunately, van der Waals molecules such as $\mathrm{Kr}_{2}$ are difficult to study experimentally, and the ZRP method is in general too crude to predict the binding energies or $Z_{\text {eff }}$ spectra for molecules for which the annihilation has been measured. That being said, this approach does provide an easily solvable and instructive model for resonant positron-molecule phenomena (see Sec. II.H.2).

Recently a theory has been proposed (Sanchez et al., 2009) that described vibrationally enhanced annihilation using the Feshbach projection operator formalism. It assumed that the positron is captured into a resonant electronic state that determines the subsequent vibrational dynamics. The full implications of this theory have yet to be elucidated. A similar mechanism drives many electron-molecule attachment processes. However, in the case of the positron, there is no experimental evidence to date of the required resonant electronic states.

\section{Positron-molecule binding}

Calculation of positron binding has proven to be exceedingly challenging. The electrostatic interaction between positrons and neutral atoms or molecules (without large dipole moments) is dominated by the nuclear repulsion. At large separations, the electric field of the positron gives rise to the attractive $-\alpha_{d} / 2 r^{4}$ potential. At short range, there is an additional attraction due virtual Ps formation (Dzuba et al., 1995; Gribakin and Ludlow, 2004). Together with polarization, these forces can overcome the static repulsion and thus enable the formation of virtual levels or bound states.

In the case of atoms, reliable calculations for positron bound states have been done for about ten species with one or two valence electrons (such as $\mathrm{Li}, \mathrm{Be}, \mathrm{Na}, \mathrm{Mg}$, $\mathrm{Cu}, \mathrm{Zn}$, etc.). The work by Mitroy et al. (2002) is a good review of the state of the field a few years ago. The calculations were done using a variety of methods: many-body theory and its combination with the configuration interaction (CI) (Dzuba et al., 1995, 1999), stochastic variational method (SVM) (Ryzhikh and Mitroy, 


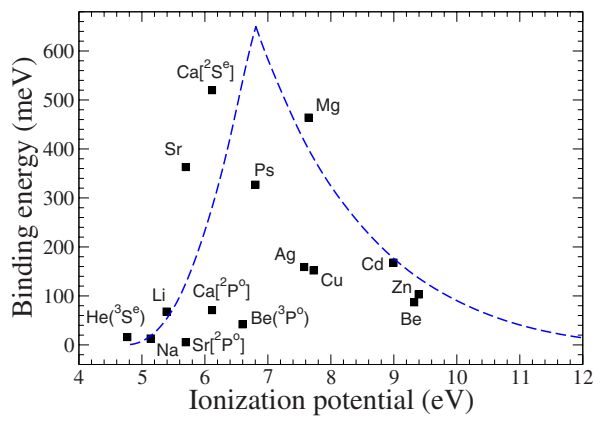

FIG. 7. (Color online) Binding energies of positron-atom complexes as a function of their ionization potential: squares, SVM and CI calculations (Mitroy et al., 2002; Bromley and Mitroy, 2007, 2010); dashed curve, model "alkali atom" (Mitroy et al., 1999). For positron binding to metastable states the latter are indicated in round brackets. The two bound states for $\mathrm{Ca}$ and $\mathrm{Sr}$ are shown in square brackets.

1997; Ryzhikh et al., 1998), and the CI method with core polarization potentials (Mitroy and Ryzhikh, 1999; Bromley and Mitroy, 2000).

These calculations provide useful insights into the physics of positron binding. One important parameter is the ionization potential of the atomic system $E_{i}$ and its relation to the Ps binding energy $E_{\mathrm{Ps}}$. For systems with $E_{i}>E_{\mathrm{Ps}}$, the electrons are relatively tightly bound in the target. Since the positron is repelled by the atomic core, it then forms a loosely bound state and stays outside the atom (i.e., represented asymptotically as $A+e^{+}$). For $E_{i}$ $<E_{\mathrm{Ps}}$ however, the positron can attract a valence electron forming a "Ps cluster" (Ryzhikh and Mitroy, 1998). In this case, the bound state is asymptotically a Ps atom orbiting the residual positive ion $\left(A^{+}+\mathrm{Ps}\right)$. Figure 7 shows the calculated binding energies $\varepsilon_{b}$ for atoms as a function of $E_{i}$. A calculation for a model "alkali atom" (Mitroy et al., 1999) shown by a dashed curve suggests that $\varepsilon_{b}$ peaks at $E_{i}=E_{\mathrm{Ps}}$, and the calculations for real atoms generally support this picture.

In the alkali atom model, a positron and a single valence electron move in the field of a fixed atomic core. As the core potential is varied, both the ionization potential and the dipole polarizability change in such a way that $\alpha_{d} \propto E_{i}^{-2}$ (Mitroy et al., 1999). In real systems, and in particular, in molecules, $\alpha_{d}$ and $E_{i}$ can be regarded more akin to independent parameters. As shown later (see Sec. VI), both are found experimentally to influence the observed binding energy. Theoretical studies of atoms and experimental studies of molecules indicate that their maximum binding energies are comparable, $\varepsilon_{b} \leqq 0.5 \mathrm{eV}$, while the values for the strongly polar alkali hydrides can be as large as $1 \mathrm{eV}$ (see below).

A major difference between positron binding to atoms and to molecules is that molecules can have permanent dipole moments. Theoretically, a static molecule with dipole moment $\mu>\mu_{\mathrm{cr}}=1.625 \mathrm{D}=0.639$ a.u. possesses an infinite number of positronic, as well as electronic bound states (Crawford, 1967). For a molecule that is free to rotate, the critical dipole moment for binding is greater than $\mu_{\mathrm{cr}}$. This critical value increases as the molecular moment of inertia decreases or the angular momentum of the molecule increases (Garrett, 1971).

At present, positron-molecule binding has been predicted theoretically for a few strongly polar molecules (Schrader and Wang, 1976; Kurtz and Jordan, 1981; Danby and Tennyson, 1988; Strasburger, 1996, 1999, 2001, 2004; Bressanini et al., 1998; Mella et al., 2001; Schrader and Moxom, 2001; Tachikawa et al., 2001, 2003; Buenker et al., 2005, 2006; Pichl et al., 2005; Chojnacki and Strasburger, 2006; Gianturco et al., 2006; Adamson et al., 2008). These calculations employed a variety of methods such as Hartree-Fock (HF), CI, and diffusion Monte Carlo (DMC), as well as explicitly correlated Gaussians (ECG). Positron bound states have also been found using the CPP method for the nonpolar cagelike molecule $\mathrm{C}_{20}$, which has a large $\alpha_{d}=25.4 \AA^{3}$ (Carey et al., 2008). A representative selection of recent results is given in Table IV. For simple molecules, such as $\mathrm{LiH}$, the results obtained by different methods are generally in good agreement. The binding energies obtained at the static HF level increase considerably when correlations are included [see, e.g., $\varepsilon_{b}$ values for $\mathrm{HCN}$, urea $\left(\mathrm{NH}_{2}\right)_{2} \mathrm{CO}$, and acetone $\left.\left(\mathrm{CH}_{3}\right)_{2} \mathrm{CO}\right]$. In fact, the current $\mathrm{CI}$ values for the larger polyatomics may still considerably underestimate the true $\varepsilon_{b}$ values due to incomplete CI expansions.

The positron density in bound states with polar molecules is asymmetric, with a strong pileup outside the negatively charged end of the molecule (Strasburger, 1999, 2001; Buenker et al., 2007). This is shown by Fig. 8 for $\mathrm{LiH}$. This figure also shows that correlations (e.g., included through ECG) produce a large increase in the positron density at the molecule, compared to the static HF calculation. This increase in the density follows the increase in $\varepsilon_{b}$ as described by Eq. (36) for bound states with nonpolar species.

Both the electron and positron densities change little with vibrational excitation of the molecule (Gianturco et al., 2006). In the alkali hydrides, positron binding noticeably increases the bond lengths and softens the vibrational modes. For example, the energies of the first vibrational excitations in $\mathrm{LiH}$ and $e^{+} \mathrm{LiH}$ are 168 and $109 \mathrm{meV}$, respectively (Mella et al., 2000; Gianturco et al., 2006). This is related to the fact that the structure of this molecule is closer to $\mathrm{Li}^{+} \mathrm{HPs}$ than to a loosely bound positron orbiting the neutral molecule. In contrast, in $\mathrm{BeO}$ and $\mathrm{MgO}$, positron binding changes the bond lengths and the vibrational frequencies by less than 1\% (Buenker et al., 2007; Buenker and Liebermann, 2008).

At present there are no $a b$ initio calculations of positron binding to alkanes or other nonpolar or weakly polar molecules (except for $\mathrm{C}_{20}$ ). Positron binding in such systems is exclusively due to electron-positron correlation effects. To obtain a bound state, the calculation must include them accurately, since binding does not exist at the static (e.g., HF) level. As an alternative, and given the availability of experimental data (Secs. V and 
TABLE IV. Calculated positron-molecule binding energies.

\begin{tabular}{|c|c|c|c|c|}
\hline Molecule & $\begin{array}{l}\mu^{\mathrm{a}} \\
\text { (D) }\end{array}$ & $\begin{array}{c}\varepsilon_{b} \\
(\mathrm{meV})\end{array}$ & Method & Reference \\
\hline \multirow[t]{5}{*}{$\mathrm{LiH}$} & \multirow[t]{5}{*}{5.88} & 1000 & DMC & Mella et al. (2000) \\
\hline & & 909 & FCSVM $^{b}$ & $\mathrm{MR}^{\mathrm{c}}$ \\
\hline & & $1005^{\mathrm{d}}$ & ECG & Strasburger (2001) \\
\hline & & $1043^{\mathrm{e}}$ & ECG & $\mathrm{BA}^{\mathrm{c}}$ \\
\hline & & 1626 & $\mathrm{NEO}^{\mathrm{b}}$ & Adamson et al. (2008) \\
\hline $\mathrm{LiF}$ & 6.33 & 599 & DMC & Mella et al. (2001) \\
\hline \multirow[t]{2}{*}{$\mathrm{BeO}$} & \multirow[t]{2}{*}{6.26} & 680 & $\mathrm{DMC}$ & Mella et al. (2001) \\
\hline & & 375 & $\mathrm{MRD}-\mathrm{CI}^{\mathrm{b}}$ & Buenker et al. (2007) \\
\hline $\mathrm{NaH}$ & 6.40 & 1031 & MRD-CI & Gianturco et al. (2006) \\
\hline $\mathrm{RbH}$ & 9.03 & 1639 & MRD-CI & Gianturco et al. (2006) \\
\hline $\mathrm{MgO}$ & 6.42 & 472 & MRD-CI & $\mathrm{BL}^{\mathrm{c}}$ \\
\hline $\mathrm{LiO}$ & 6.84 & 304 & MRD-CI & $\mathrm{BL}^{\mathrm{c}}$ \\
\hline $\mathrm{CH}_{2} \mathrm{O}$ & 2.33 & 19 & $\mathrm{CI}$ & Strasburger (2004) \\
\hline \multirow[t]{3}{*}{$\mathrm{HCN}$} & \multirow[t]{3}{*}{2.98} & 2 & $\mathrm{HF}$ & $\mathrm{CS}^{\mathrm{c}}$ \\
\hline & & 35 & $\mathrm{CI}$ & $\mathrm{CS}^{\mathrm{c}}$ \\
\hline & & 38 & DMC & Kita et al. (2009) \\
\hline \multirow[t]{2}{*}{ Urea } & \multirow[t]{2}{*}{3.99} & 6 & $\mathrm{HF}$ & Tachikawa et al. (2003) \\
\hline & & 13 & $\mathrm{CI}$ & Tachikawa et al. (2003) \\
\hline \multirow[t]{2}{*}{ Acetone } & \multirow[t]{2}{*}{2.88} & 1 & $\mathrm{HF}$ & Tachikawa et al. (2003) \\
\hline & & 4 & $\mathrm{CI}$ & Tachikawa et al. (2003) \\
\hline \multirow[t]{2}{*}{$\mathrm{C}_{20}$} & & $780^{f}$ & $\mathrm{CPP}$ & Carey et al. (2008) \\
\hline & & $230-250^{f}$ & $\mathrm{CPP}$ & Carey et al. (2008) \\
\hline
\end{tabular}

${ }^{a}$ Dipole moments from Gutsev et al. (1997), Lide (2000), and Buenker et al. (2005) or as cited.

${ }^{\mathrm{b}}$ FCSVM, fixed-core SVM; NEO, nuclear-electronic orbital method; MRD-CI, multireference single- and doubleexcitation CI.

${ }^{\mathrm{c}}$ Mitroy and Ryzhikh (2000) (MR); Bubin and Adamowicz (2004) (BA); Chojnacki and Strasburger (2006) (CS); Buenker and Liebermann (2008) (BL).

${ }^{\mathrm{d}}$ Adiabatic positron affinity.

${ }^{\mathrm{e}}$ Non-Born-Oppenheimer variational calculation.

${ }^{\mathrm{f}}$ The $C_{i}$ isomer of $\mathrm{C}_{20}$ is predicted to have a deeply bound $s$-type state and three weakly bound $p$-type states.

VI), Gribakin and Lee (2006b, 2009) explored positronmolecule binding to alkanes using ZRP. The ZRP is the simplest form of a model potential, suited to studying low-energy processes (Demkov and Ostrovsky, 1988). The idea of the model potential approach is to fit the potential to experimental data (e.g., the binding energy for a given molecule) and then use it to study binding for a range of similar molecules.

In the ZRP method, the bound-state wave function of the positron in the field of $N$ centers placed at $\mathbf{R}_{i}$ has the form (Demkov and Ostrovsky, 1988)

$$
\Psi(\mathbf{r})=\sum_{i=1}^{N} A_{i} \frac{e^{-\kappa\left|\mathbf{r}-\mathbf{R}_{i}\right|}}{\left|\mathbf{r}-\mathbf{R}_{i}\right|}
$$

where $\kappa>0$ is related to the bound-state energy by $\varepsilon_{0}$ $=-\kappa^{2} / 2$. The interaction with each center is param-

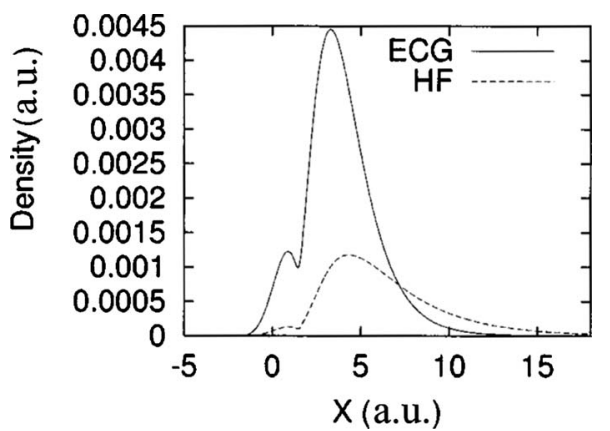

FIG. 8. Positron density in $e^{+} \mathrm{LiH}$ along the molecular axis: dashed curve, HF $\left(\varepsilon_{b}=0.16 \mathrm{eV}\right)$; solid curve, ECG $\left(\varepsilon_{b}\right.$ $=0.94 \mathrm{eV}) ; \mathrm{Li}$ atom is at $X=-1.51$ a.u., and $\mathrm{H}$ at $X=1.51$ a.u. From Strasburger, 1999.

etrized by $\kappa_{0 i}$ through the boundary condition

$$
\left.\Psi\right|_{\mathbf{r} \rightarrow \mathbf{R}_{i}} \simeq \text { const } \times\left(\left|\mathbf{r}-\mathbf{R}_{i}\right|^{-1}-\kappa_{0 i}\right)
$$

Subjecting $\Psi$ from Eq. (51) to $N$ conditions (52) yields a set of linear homogeneous equations for $A_{i}$, whose solvability determines the allowed values of $\kappa$.

The alkanes $\mathrm{C}_{n} \mathrm{H}_{2 n+2}$ were modeled by a planar zigzag chain of $n$ ZRPs, each representing the $\mathrm{CH}_{3}$ or $\mathrm{CH}_{2}$ group. The distance between the neighboring ZRPs is given by the length of the C-C bond 2.91 a.u., and the angle between adjacent bonds is equal to $113^{\circ}$. The parameter $\kappa_{0 i}=-0.69$ a.u. was chosen to reproduce the binding energy for dodecane $(n=12) \varepsilon_{0}=-220 \mathrm{meV}$ (Gribakin and Lee, 2009). Figure 9 compares the results of this calculation with the measured binding energies for alkanes up to $n=16$ (cf. Table V).

Figure 9 shows that the model gives a good overall description of positron binding to alkanes, including the prediction of the second bound state. However, it fails to capture in a quantitative way some of the details. The model predicts binding for $n \geqslant 4$, whereas ethane $(n=2)$ is observed to bind positrons. The model also predicts that a second bound state emerges for $n=13$, while experimentally this state is observed at $n=12$ (Barnes et al., 2006; Young and Surko, 2008b); cf. Fig. 25.

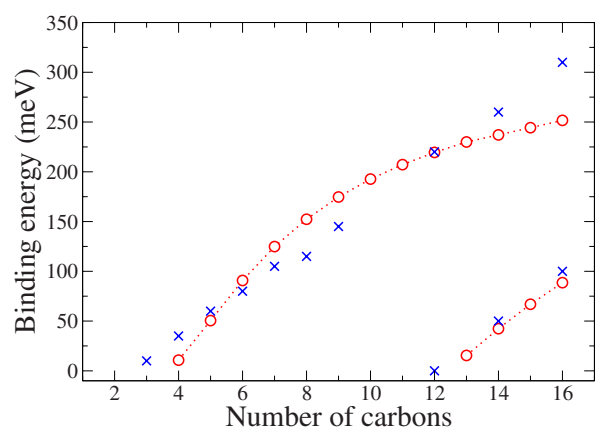

FIG. 9. (Color online) Positron binding energies for alkanes from experiment (crosses) (Table V) and ZRP model calculation (circles). The ZRP model is fit to $\varepsilon_{b}=220 \mathrm{meV}$ for dodecane. From Gribakin and Lee, 2009. 

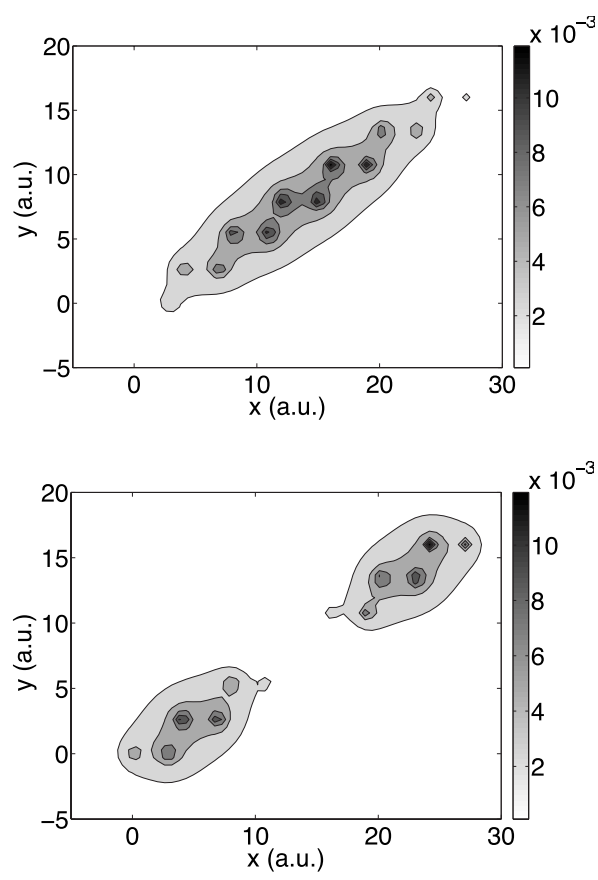

FIG. 10. Two-dimensional density of the positron wave functions in the ZRP model for the first (top) and second (bottom) bound states in tetradecane $(n=14)$. In these plots, the first carbon atom is at the origin, and the $\mathrm{C}$ - $\mathrm{C}$ bonds are alternately parallel and at $67^{\circ}$ to the $x$ axis. From Gribakin and Lee, 2009.

To visualize the bound states, the two-dimensional density

$$
\rho(x, y)=\int_{-\infty}^{+\infty}|\Psi(x, y, z)|^{2} d z
$$

where $x$ and $y$ are in the plane of the carbon chain, is shown in Fig. 10 for the first and second bound states of tetradecane $(n=14)$. Both states are quite diffuse, with the positron spread over the whole molecule. The wave function of the second bound state must be orthogonal to the ground state, and so it changes sign on a nodal surface close to the center of the molecule. On the density plot (Fig. 10, bottom), this corresponds to an area of low density near the midpoint. The actual positron wave functions are expected to differ from that given by Eq. (51) in that the latter does not exclude the positron from the regions inside the atomic cores (which are of "zero range" in the model). However, the atomic cores are relatively small compared to the extent of the positron wave function, in keeping with the main assumption of the ZRP model. Thus the model captures the main features of positron-molecule bound states.

\section{EXPERIMENTAL TOOLS AND PROCEDURES}

In this section, experimental techniques are described that have been used to investigate low-energy positronmolecule annihilation and related effects. The methodology has varied considerably over the past half century of these studies, and no attempt is made to be complete.
Emphasis is placed upon typical and/or best practices. The interested reader is referred to the original papers for further details.

\section{A. Annihilation-rate measurements with thermalized positrons in atmospheric pressure gases}

Positrons from conventional sources such as radioisotopes or electron accelerators typically have energies ranging from $\sim 1 \mathrm{keV}$ to $\sim 0.5 \mathrm{MeV}$. Thus, to study positron interactions at low energies, some method must be used to slow the positrons. Early annihilation rate measurements were done using the test species themselves as "moderators" and measuring the spectra of time delays between the positron production and annihilation signals (Shearer and Deutsch, 1949; Deutsch, 1951a, 1951b; Osmon, 1965a; Griffith and Heyland, 1978; Heyland et al., 1982; Wright et al., 1983). In a typical experiment, the $1.28 \mathrm{MeV}$ gamma ray that accompanies positron emission from a ${ }^{22} \mathrm{Na}$ positron source provides a start signal, and the detection of a single, $511 \mathrm{keV}$ gamma ray from a two-gamma annihilation event is used as a stop signal. Achieving an adequate signal-to-noise ratio requires working at gas densities $\geqslant 0.1$ amagat.

Annihilation rates are obtained from the dependence of the exponential time decay of the $511 \mathrm{keV}$ annihilation signal (i.e., after the positrons come to thermal equilibrium) on the test-gas density. Using fast electronics, it was also possible to measure the slowing down of the fast positrons (Sharma and McNutt, 1978). Where necessary, the rate of thermalization was increased by adding a light species with a small $Z_{\text {eff }}$ value such as molecular hydrogen (Wright et al., 1985), and the longtime ortho-positronium component was quenched using a small admixture of a gas with unpaired electron spins such as NO or $\mathrm{O}_{2}$ (Deutsch, 1951a, 1951b).

\section{B. Buffer-gas positron traps as tailored sources of positrons}

Buffer-gas (BG) traps (Surko, Passner, et al., 1988; Murphy and Surko, 1992; Surko et al., 1999) proved to be a useful tool to tailor positron gases, plasmas, and beams for positron annihilation studies (Surko and Greaves, 2004). Positrons from a sealed ${ }^{22} \mathrm{Na}$ radioactive source are slowed to electron-volt energies using a solidneon reflection moderator [efficiency 1-2\% (Greaves and Surko, 1996)]. A $50 \mathrm{mCi}^{22} \mathrm{Na}$ source and neon moderator produces $\sim(5-10) \times 10^{6}$ slow positrons per second. The slow positrons are then guided magnetically into a buffer-gas Penning-Malmberg trap (Murphy and Surko, 1992). It consists of a uniform magnetic field ( $B$ $\sim 0.1 \mathrm{~T})$ coaxial with a set of cylindrical electrodes biased to form a stepped three-stage potential well. The stages contain a nitrogen buffer gas with successively lower pressures. Positrons become trapped by losing energy through electronic excitations of the $\mathrm{N}_{2}$ molecules. They then cool to the ambient (i.e., room) temperature in the third stage by additional collisions with the $\mathrm{N}_{2}$. Recently a small amount of $\mathrm{CF}_{4}$ was added to increase 


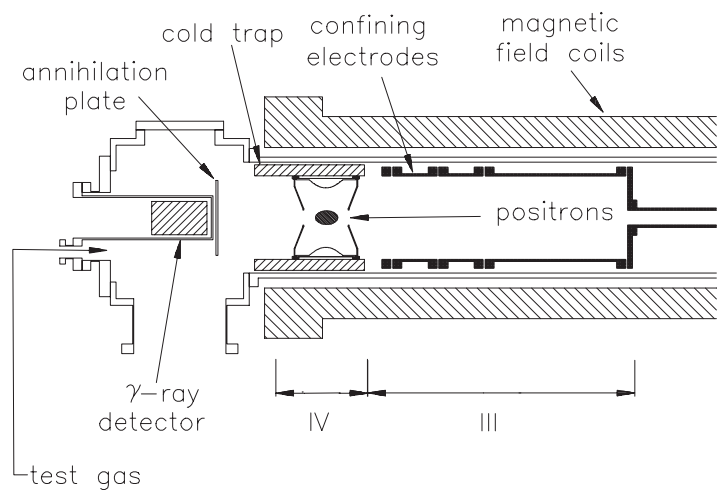

FIG. 11. Schematic diagram of the apparatus used to study the interaction of trapped, thermal positrons with low-pressure gases. The positrons are contained in a fourth trapping stage surrounded by a cold surface to minimize the effects of impurities present in the vacuum system. From Iwata et al., 1995. From Gribakin and Lee, 2009.

the cooling rate (Greaves and Surko, 2000; Sullivan, Gilbert, Marler, Greaves, et al., 2002). The result is a magnetized thermal gas (or plasma) of as many as $10^{8}$ positrons at a temperature of $300 \mathrm{~K}$. The trapped positrons are in a background gas pressure $\leqslant 10^{-6}$ torr, which can then be pumped out depending upon the experiment.

\section{Annihilation-rate measurements in positron traps}

Positron traps have enabled an improved method to study the interaction of thermal positrons with a large variety of test species (Surko, Passner, et al., 1988; Murphy and Surko, 1991; Iwata et al., 1995, 2000). In this case, the positrons are trapped, the buffer gas is pumped out, and a test-gas species is introduced into the trap at a low pressure. Low pressures ensure that the annihilation events are due to two-body positron-molecule interactions and thus that three-body processes are negligible. This technique also permitted study of low vapor pressure targets. Furthermore, the positron temperature (e.g., $300 \mathrm{~K}$ ) could be measured to verify that they are in thermal equilibrium with the test species. This was done using standard plasma techniques, dumping the positrons, and measuring their energy distribution using a retarding potential analyzer (Murphy and Surko, 1992).

The apparatus for these thermal annihilation-rate measurements is shown in Fig. 11 (Iwata et al., 1995). In this case, the trapped positrons were shuttled to a separate confinement stage surrounded by a cryogenically cooled surface to reduce the level of impurities in the vacuum system. It operated with liquid nitrogen $(77 \mathrm{~K})$ or an ethanol-water mixture $\left(-7^{\circ} \mathrm{C}\right)$ depending upon the test species. The annihilation was monitored by holding the positrons for a given time, then dumping those that had not annihilated onto a collector plate, and measuring the gamma-ray signal using a $\mathrm{NaI}(\mathrm{Tl})$ scintillator and a photomultiplier. Experiments were also conducted in which the thermal positrons were heated by short bursts of radio-frequency noise to provide a mea- sure of the annihilation rate as a function of positron temperature (Iwata et al., 2000).

For test species that are gases at $300 \mathrm{~K}$, the pressure is controlled to microtorr precision by feedback using a capacitance manometer and a piezoelectric valve. A liquid delivery system, while offering less control, permitted studies of a broader range of test species. It can be used with species that have relatively low boiling points (i.e., close to room temperature) and pulverized solid samples, such as naphthalene (Young, 2007). For liquid samples, a freeze-pump-thaw procedure is used to eliminate volatile contaminants. The sample is placed in a temperature-regulated bath, and a needle valve is used to leak vapor into the annihilation cell. While there was no feedback control of the pressure, it was still reasonably stable (Iwata et al., 1995).

Some vapors must be run at pressures below the sensitivity of the capacitance manometer to avoid detector saturation. In this case, the pressure of the test species is measured with an ion gauge calibrated against the manometer at higher pressures. A few species studied (e.g., naphthalene) have such low vapor pressures that accurate pressure calibration was not possible (Young, 2007; Young and Surko, 2008b).

The major uncertainty in these $Z_{\text {eff }}$ measurements is estimated to be a $\pm 20 \%$ systematic error in the measurement of test-gas pressures.

\section{Trap-based cold positron beams}

To obtain detailed information about positron interactions with matter, the tool of choice is a beam of positrons with a well defined and tunable energy. In early experiments, the beam energy resolution was limited by the energy spread of positrons emerging from a moderator (e.g., a fraction of an electron volt). While the use of a cold primary moderator (Brown et al., 1986) or remoderator (Gullikson and Mills, 1987) could reduce the energy spread to $30-40 \mathrm{meV}$, these techniques were rarely, if ever, used in atomic physics experiments. The advent of the BG positron accumulator enabled an efficient, pulsed, and tunable low-energy positron beam with a comparable, and potentially smaller energy spread (Gilbert et al., 1997; Kurz et al., 1998; Gilbert, 2000).

In this technique, $\sim 10^{4}$ positrons are accumulated in a time $\sim 0.1 \mathrm{~s}$ and cooled for a similar time (Gilbert et al., 2002; Sullivan et al., 2004). Then the exit-gate electrode is lowered to a potential $V_{E}$, which sets the beam energy. The bottom of confining potential well is then raised (i.e., to $\sim 0.25 \mathrm{~V}$ above $V_{E}$ ) in a time $\sim 5 \mu$ s to produce a positron pulse with a similar time duration. The beamtransport energy (typically $\sim 2 \mathrm{eV}$ ) is set below the threshold for positronium formation to avoid positron loss and extraneous gamma-ray signals. All this is done in the BG-trap magnetic field (i.e., $B \sim 0.15 \mathrm{~T}$ ), producing pulses of $(1-3) \times 10^{4}$ positrons at a few $\mathrm{Hz}$ rate. The pulses are magnetically guided to the annihilation cell in fields ranging from 0.03 to $0.1 \mathrm{~T}$. 
The beam energy distribution is measured using the cylindrical electrode of the gas cell as a retarding potential analyzer (RPA) (Young, 2007). The mean energy of the beam in the annihilation cell can be verified independently by measuring the time-of-flight delay of the positrons passing through the cell as a function of increasing cell potential $V_{C}$ (Sullivan, Gilbert, Marler, Greaves, et al., 2002; Marler, 2005). Typical uncertainties in the mean beam energy are $\sim 10 \mathrm{meV}$.

The parallel energy distribution can be modeled by a single Gaussian (Young, 2007). At a more accurate level, there is typically a high-energy tail containing $\sim 10 \%$ of the beam particles, depending upon the beam-formation protocol and the relative magnitudes of the magnetic field in the buffer-gas trap and the measurement region. ${ }^{19}$ Parallel energy spreads as small as $18 \mathrm{meV}$ [full width at half maximum (FWHM)] can be achieved using this technique (Gilbert et al., 1997) when the beam is kept in the same strength magnetic field as the buffergas positron accumulator. Typical values of parallel energy spread in the annihilation experiments described here are somewhat larger, $25-30 \mathrm{meV}$, due to the fact that the annihilation cell was at a lower magnetic field than that of the trap (Barnes et al., 2003). The spread in transverse energies in the BG trap is set by the ambient temperature $T_{\perp}=25 \mathrm{meV}$ (i.e., corresponding to an electrode temperature of $\sim 300 \mathrm{~K}$ ).

The positron energy distribution in the beam is modeled as

$$
f\left(\varepsilon_{\|}, \varepsilon_{\perp}\right)=\frac{1}{k_{B} T_{\perp} \sqrt{2 \pi \sigma^{2}}} \exp \left[-\frac{\varepsilon_{\perp}}{k_{B} T_{\perp}}-\frac{\left(\varepsilon_{\|}-\bar{\varepsilon}\right)^{2}}{2 \sigma^{2}}\right],
$$

where $\varepsilon_{\|}$and $\varepsilon_{\perp}$ are the parallel and transverse positron energies, $\sigma$ is the root-mean-squared width of the parallel energy distribution (i.e., corresponding to a FWHM of $\sigma \sqrt{8 \ln 2}$ ), and $\bar{\varepsilon}$ is the mean parallel energy of the positron beam.

This distribution has been verified using the resonant annihilation peak for the $\mathrm{C}-\mathrm{H}$ stretch modes in propane (cf. Sec. V). The linewidth of the VFR peak is assumed to be negligible (i.e., $\lesssim 1 \mathrm{meV}$; cf. Sec. II), as is the spread of the propane $\mathrm{C}-\mathrm{H}$ stretch frequencies. The distribution in Eq. (54) is convolved with a delta function, yielding the distribution of (total) positron energies of the beam (Gribakin and Lee, 2006a),

$$
f_{\mathrm{b}}(\varepsilon-\bar{\varepsilon})=\int f\left(\varepsilon_{\|}, \varepsilon_{\perp}\right) \delta\left(\varepsilon_{\|}+\varepsilon_{\perp}-\varepsilon\right) d \varepsilon_{\|} d \varepsilon_{\perp}
$$

\footnotetext{
${ }^{19}$ The ratio $\varepsilon_{\perp} / B$ and the total positron energy $\varepsilon=\varepsilon_{\|}+\varepsilon_{\perp}$ are both conserved in this magnetic beam-transport system, where $\varepsilon_{\|}$is the parallel beam energy and $\varepsilon_{\perp}$ is the transverse energy of the particles' gyromotion in the plane perpendicular to the magnetic field $B$ (Sullivan, Gilbert, Marler, Greaves, et al., 2002; Barnes et al., 2003).
}

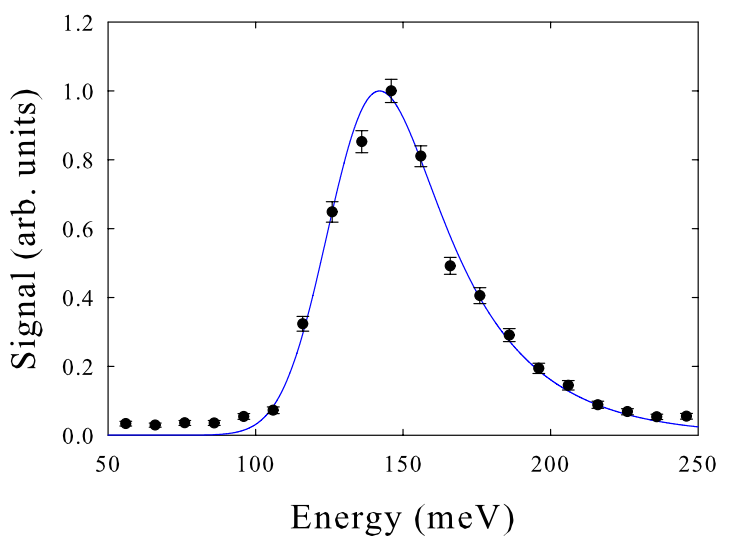

FIG. 12. (Color online) Measurement of the distribution of total positron energies in the trap-based beam using a vibrational Feshbach annihilation resonance: solid curve, prediction of Eq. (56), normalized arbitrarily, shifted in energy, and fitted to the energy-reversed, normalized, C-H stretch peak in $Z_{\text {eff }}$ for propane $(\bullet)$, with $T_{\perp}=26 \mathrm{meV}$ and a parallel energy spread (FWHM) of $27 \mathrm{meV}$. From Young, 2007.

$$
\begin{aligned}
= & \frac{1}{2 k_{B} T_{\perp}} \exp \left[\frac{\sigma^{2}}{2\left(k_{B} T_{\perp}\right)^{2}}\right] \exp \left(-\frac{\varepsilon-\bar{\varepsilon}}{k_{B} T_{\perp}}\right) \\
& \times\left\{1+\Phi\left(\frac{1}{\sqrt{2}}\left(\frac{\varepsilon-\bar{\varepsilon}}{\sigma}-\frac{\sigma}{k_{B} T_{\perp}}\right)\right)\right\},
\end{aligned}
$$

where $\Phi(x)$ is the error function. Note that this energy distribution depends on the difference between the total energy $\varepsilon$ and mean parallel energy $\bar{\varepsilon}$.

The resulting fit shown in Fig. 12 is in excellent agreement with the data. Due to the spread in perpendicular energies, the position of the peak observed as a function of the mean parallel energy (which is set and measured by varying $V_{C}$ ) is about $12 \mathrm{meV}$ below the true energy of the peak (i.e., as a function of the total positron energy). The annihilation spectra in this review are presented as a function of the total positron energy, which is taken to be $12 \mathrm{meV}$ higher than the mean parallel energy set in the experiment.

\section{E. Energy-resolved annihilation measurements}

Central to this review are measurements of positron annihilation on molecules, resolved as a function of incident positron energy (Gilbert et al., 2002; Barnes et al., 2003, 2006; Young and Surko, 2008b). The annihilation cell is shown schematically in Fig. 13. It consists of a cylindrical gold-plated electrode $4.4 \mathrm{~cm}$ in diameter and $17 \mathrm{~cm}$ long. The gamma-ray detector and associated shielding restrict the detector field of view to a region $\leqslant 15 \mathrm{~cm}$ in length along the axis of the cell. Magnet coils outside the cell impose a field of $\sim 0.075-0.095 \mathrm{~T}$, with the lowest value in region viewed by the detector. Metal baffles shield the gas cell and the detector from spurious gamma-ray decays. The system for handling gases and vapors is described in Sec. III.C above.

Single gamma rays are detected using a CsI crystal and a photodiode, followed by a single-channel analyzer 


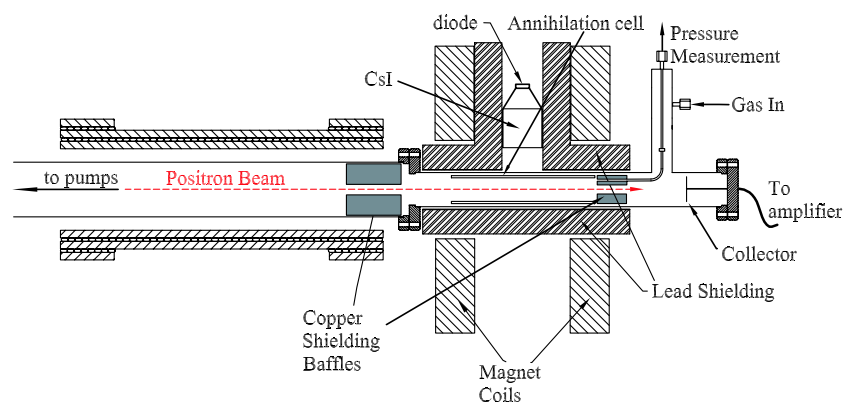

FIG. 13. (Color online) Schematic diagram of the gas cell, shielding, and detection apparatus used for energy-resolved positron annihilation measurements (not to scale). From Young, 2007.

centered on the $511 \mathrm{keV}$ annihilation gamma-ray line. The absolute detector efficiency and the sensitivity along the path of the positron beam are measured using a calibrated gamma-ray test source (Gilbert et al., 2002).

Pulses of positrons pass through the gas cell several times while annihilation events are recorded, with the total scattering kept below $15 \%$. A typical time window for this measurement is $\sim 15 \mu$ s (Young and Surko, 2008b). Positrons are kept in flight while the annihilation events are recorded to avoid spurious gamma-ray background signals. To avoid detector saturation, the average signal level is adjusted to $\sim 1$ count per 10 positron pulses. Typical test-gas pressures range from 0.1 to $100 \mu$ torr due to the large variations in annihilation rate for different chemical species. Background signals can be as low as one count per $10^{9}$ positrons cycled through the annihilation cell.

A typical spectrum consists of $\sim 10-25$ pulse-train measurements at each energy, taken at $10-15 \mathrm{meV}$ intervals over the relevant range of positron energies (e.g., $\leqslant 500 \mathrm{meV}$ ). This measurement is then repeated a few hundred times. Complications due to scattering restrict measurements to energies $\geqslant 50 \mathrm{meV}$. Absolute values of $Z_{\text {eff }}$ are obtained from measurement of the detector efficiency, the pulse strength, the detector-sensitivityaveraged path length, and the test-gas pressure.

Uncertainties in these parameters are estimated to result in a $20 \%$ overall systematic uncertainty in the absolute magnitude of $Z_{\text {eff. }}$ The error bars shown in this review indicate the statistical uncertainty due to the finite number of counts at a given energy. In many cases, these statistical errors are smaller than the size of the data points. The linearity of the signal with test-gas pressure is checked to ensure that scattering and three-body effects are negligible.

A separate annihilation cell was used for studies of annihilation as a function of the temperature of the test species (Young and Surko, 2008a). It is capable of cooling target gases down to $100 \mathrm{~K}$. To ensure that the test species are actually thermalized, the annihilation cell was operated at temperatures below the sticking temperature of the test species on the cell walls.

\section{F. Gamma-ray spectral measurements}

The center-of-mass momentum of the annihilating electron-positron pair contains important information about the momentum distribution of the bound electrons in an atomic or molecular target. It produces both Doppler shifts of the photon energies and changes in their directions (see Sec. II.B). Using such measurements, one can distinguish, for example, annihilation on different atoms or annihilation on valence and innershell electrons in a given species (Iwata et al., 1997).

In a two-gamma event, the photon momentum distribution can be determined by measuring either the angular deviation of the gamma rays [the so-called angular correlation of annihilation radiation (ACAR) technique (Coleman et al., 1994)] or the Doppler broadening of the annihilation gamma-ray line. For the tenuous samples studied here, Doppler broadening is the method of choice.

The apparatus for these measurements is shown in Fig. 11 (Iwata, Greaves, and Surko, 1997). The gamma rays are detected using a high-purity Ge detector with an energy resolution of $1.16 \mathrm{keV}$ (FWHM). Positrons are trapped and cooled, then the $\mathrm{N}_{2}$ trapping gas is pumped out, and the test gas is introduced. Gamma-ray data are recorded, and then this cycle is repeated. The errors in the measurement of gamma-ray energies are estimated to be $\pm 0.02 \mathrm{keV}$. The errors in spectral intensity are predominantly statistical due to the finite number of counts (Iwata, Greaves, and Surko, 1997).

\section{G. Annihilation-induced fragmentation}

Positron annihilation on atomic and molecular species produces a spectrum of positive ions that can be measured using time-of-flight techniques. The first experiment of this kind arranged for positrons in a Penning trap to interact with molecular species for a short time. Then the resulting ions were dumped and the mass spectrum measured using a time-of-flight technique (Passner et al., 1989; Glish et al., 1994). Subsequently, more detailed studies were conducted using improved techniques to measure the ion mass spectra (Donohue et al., 1990; Hulett et al., 1993; Xu et al., 1993, 1994, 1995; Hulett, Xu, McLuckey, Lewis, et al., 1996; Moxom et al., 2000). In this case, positrons from an electron linear accelerator were moderated and then accumulated in a Penning trap, where they were allowed to interact with the test species. The product ions were accelerated and detected using a microchannel plate. Significantly higher mass resolution was achieved using a spatially varying (quadratic) potential to arrange the same arrival time for ions of the same mass starting at different initial positions in the trap.

\section{ANNIHILATION ON SMALL MOLECULES}

Research on positron annihilation on atomic and molecular targets has typically been focused in two areas: atomic and small molecular species where modest anni- 
hilation rates are observed, and large hydrocarbons that are characterized by very large annihilation rates. Some small polyatomic molecules occupy a middle ground (Gribakin and Lee, 2006a). Here annihilation proceeds in a different manner than in other small targets due to the fact that these molecules can temporarily bind a positron via VFRs. Further, in contrast to larger hydrocarbons, the theoretical description of annihilation in these targets is relatively simple. The focus of this section is VFR-mediated annihilation in small molecules and the contrasting case of annihilation in small molecules that do not bind positrons.

Energy-resolved $Z_{\text {eff }}$ spectra, such as those shown in Fig. 1, reveal peaks corresponding to the resonant transfer of energy from the positron to specific molecular vibrations. In each of these resonances the positron becomes temporarily attached to the molecule, resulting in a greatly enhanced annihilation rate. The energies of the resonances are given by Eq. (3), namely, $\varepsilon_{\nu}=E_{\nu}-\varepsilon_{b}$, where $E_{\nu}$ is the energy of the excited vibration $\nu$ and $\varepsilon_{b}$ is the positron-molecule binding energy. Fundamental vibrations, for which $E_{\nu}=\omega_{\nu}$, can produce strong annihilation. As a result, the molecular annihilation spectra are somewhat similar to infrared-absorption spectra. However, the magnitudes of the annihilation resonances are not proportional to the IR absorption strengths. They follow a different scaling with molecular size. While IR-active modes dominate the spectra of many molecules, there are cases in which nominally IRinactive modes, as well as combinations and overtones, also appear to produce annihilation resonances.

\section{A. Halomethanes as a benchmark example of VFR}

The singly halogenated methanes are a near-perfect set of molecules to test theories of positron VFRs. Each molecule has only six vibrational degrees of freedom, all of which are dipole active. There are three energyseparated pairs of fundamental vibrations: the $\mathrm{C}-\mathrm{H}$ stretch modes, the $\mathrm{C}-\mathrm{H}$ bend modes, and the $\mathrm{C}-\mathrm{X}$ modes, where $X$ is the halogen. The energy-resolved annihilation spectra and the infrared absorption spectra for these molecules are shown in Fig. 14. In $\mathrm{CH}_{3} \mathrm{Cl}$ and $\mathrm{CH}_{3} \mathrm{Br}$, one can discern VFR from all of the infraredactive modes. The high-energy peak is due to the $\mathrm{C}-\mathrm{H}$ stretch mode, and the broad low-energy feature is due to the $\mathrm{C}-\mathrm{H}$ bend and $\mathrm{C}-\mathrm{X}$ modes. There is no evidence of multimode VFRs.

As shown in Fig. 14, the position of the C-H stretch resonance shifts downward in energy as the size of the halogen atom is increased, reflecting an increase in the positron binding energy. The binding energies range from near zero in $\mathrm{CH}_{3} \mathrm{~F}$ to about $40 \mathrm{meV}$ in $\mathrm{CH}_{3} \mathrm{Br}$. Since vibrational Feshbach resonances cannot occur unless the positron is bound to the molecule, the small positive energy shift of the $\mathrm{C}-\mathrm{H}$ stretch peak in $\mathrm{CH}_{3} \mathrm{~F}$ is likely the result of a very small binding energy and a small positive shift in the mode energy.

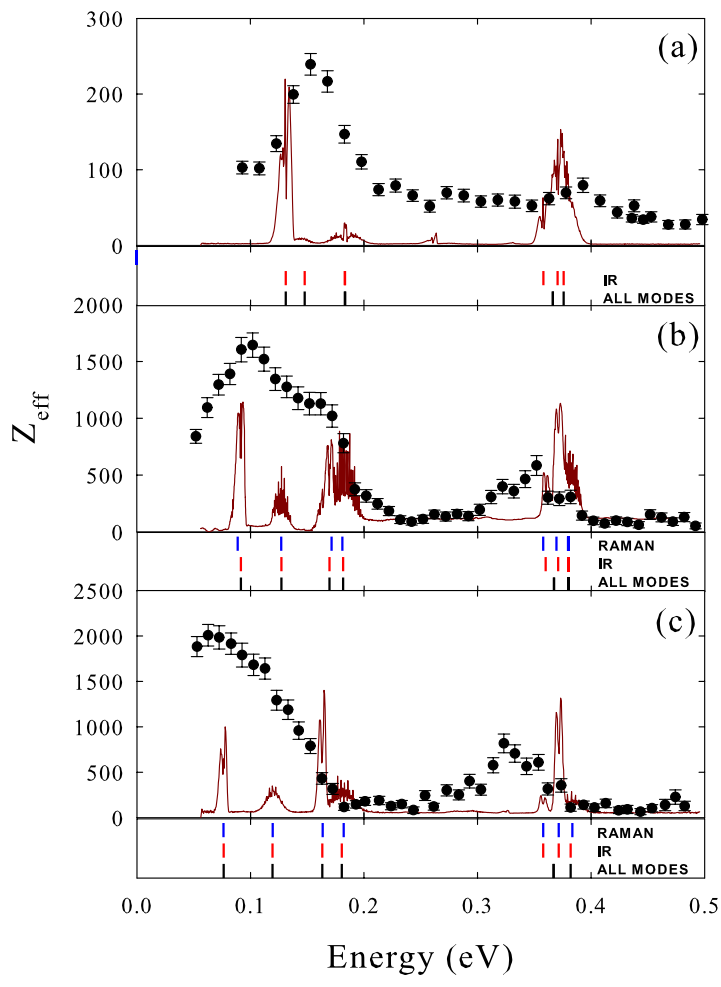

FIG. 14. (Color online) Annihilation rates $Z_{\text {eff }}(\mathbf{O})$ and IR absorption (solid curves) for methyl halides: (a) $\mathrm{CH}_{3} \mathrm{~F}$, (b) $\mathrm{CH}_{3} \mathrm{Cl}$, and (c) $\mathrm{CH}_{3} \mathrm{Br}$ (Barnes et al., 2006). Vertical bars below each plot indicate the vibrational energies from IR and Raman measurements, and selected mode frequencies ("all modes"). From Linstrom and Mallard, 2005.

According to Eq. (42), the annihilation rate in small molecules can be described by a sum of Breit-Wigner resonances for each mode, $\nu$, convolved with the instrumental positron energy resolution function $f_{\mathrm{b}}(\varepsilon)$. All of the modes are dipole active in the halomethanes. Thus the elastic capture rates, which are roughly proportional to the IR strengths, are expected to be much larger than the annihilation rates (see Sec. II.F). As a result, $\Gamma \simeq \Gamma^{e}$, and Eq. (42) is greatly simplified. Using Eq. (36), one obtains

$$
\bar{Z}_{\mathrm{eff}}^{(\mathrm{res})}(\bar{\varepsilon})=\pi F \sum_{\nu} g_{\nu} b_{\nu} \Delta\left(\bar{\varepsilon}-\varepsilon_{\nu}\right),
$$

where $g_{\nu}=\sqrt{\varepsilon_{b} / \varepsilon_{\nu}}$. Since $F$ is assumed to be constant (see Sec. II.D), the relative magnitudes of the dipole-active resonances in a given small molecule are determined by the $g_{\nu}$ factors. The only adjustable parameter is the binding energy, which can be determined by comparison with experiment. The application of Eq. (57) to the halomethanes and their deuterated counterparts is shown in Fig. 15. The small contribution from nonresonant direct annihilation, described by Eq. (29), is included.

For the two larger halogens, the binding energy was obtained from the position of the $\mathrm{C}-\mathrm{H}$ stretch peaks, while for $\mathrm{CH}_{3} \mathrm{~F}$, the (small) binding energy was determined by fitting to the magnitude of $Z_{\text {eff }}$ (Gribakin and Lee, 2006a). The resulting binding energies for $\mathrm{CH}_{3} \mathrm{~F}$, 


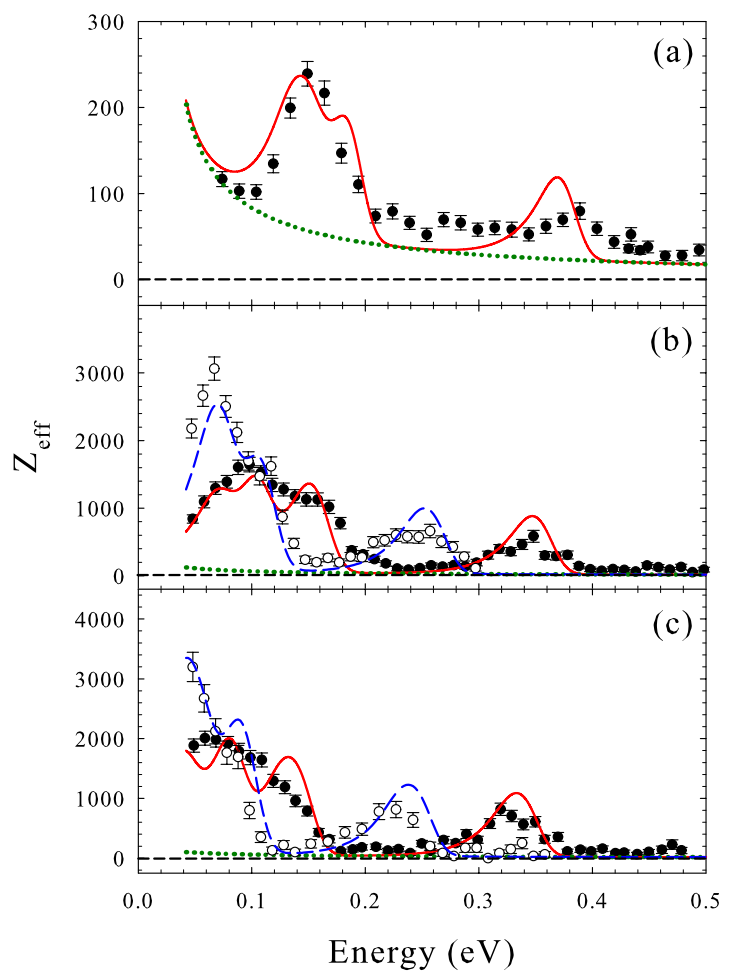

FIG. 15. (Color online) Comparison of experimental and theoretical $Z_{\text {eff }}$ for methyl halides $\mathrm{CH}_{3} X$ ( and solid curves) and $\mathrm{CD}_{3} X$ ( $\bigcirc$ and dashed curves) for (a) $X=\mathrm{F}, \varepsilon_{b}=0.3 \mathrm{meV}$; (b) $X=\mathrm{Cl}, \varepsilon_{b}=25 \mathrm{meV}$; and (c) $X=\mathrm{Br}, \varepsilon_{b}=40 \mathrm{meV}$ (Barnes et al., 2006; Gribakin and Lee, 2006a; Young et al., 2008). Dotted curves show the contributions of direct annihilation.

$\mathrm{CH}_{3} \mathrm{Cl}$, and $\mathrm{CH}_{3} \mathrm{Br}$ are $0.3,25$, and $40 \mathrm{meV}$, respectively, and the agreement between the theoretical and experimental $Z_{\text {eff }}$ spectra is remarkably good. As the binding increases, each annihilation peak shifts downward in energy and increases in magnitude in accord with the predicted $g$-factor scaling. The theory also predicts successfully the absolute magnitudes of each of these features.

For a wide variety of hydrocarbons, experiments have established that the positron binding to the molecule is unchanged when the hydrogen atoms are replaced with deuterium atoms. This is plausible since the binding energy is expected to be primarily a function of the electronic rather than the vibrational degrees of freedom (Barnes et al., 2003). The only difference is that the deuterated species have lower vibrational energies due to the larger reduced masses associated with the C-D modes. The binding energies for the hydrogenated species were used to predict the $Z_{\text {eff }}$ spectra for the deutrated species, thus providing a stringent test of the theory. The results of this comparison are shown in Fig. 15. Note that the $g$ factors and hence the magnitudes of the peaks are larger for the deuterated species, because the resonances occur at smaller positron impact energies. The theory, now with no free parameters, works well for the deuterated halomethanes just as it did for the hydrogenated species. To our knowledge, no other theory has demonstrated such close agreement, in both

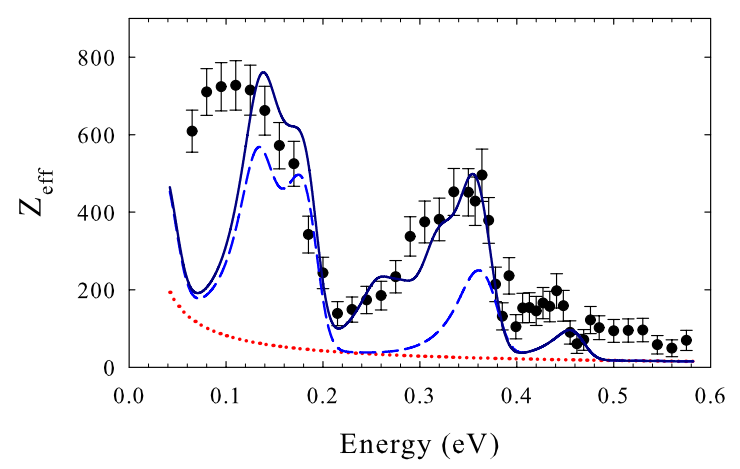

FIG. 16. (Color online) Comparison of experimental $Z_{\text {eff }}(\bullet)$ for methanol $\left(\mathrm{CH}_{3} \mathrm{OH}\right)$ with theory: dotted curve, contribution of direct annihilation $Z_{\text {eff }}^{\text {(dir) }}$; dashed curve, total $Z_{\text {eff }}$ due to VFR of vibrational fundamentals for $\varepsilon_{b}=2 \mathrm{meV}$; solid curve, total $Z_{\text {eff }}$ due to resonant annihilation involving the 12 modes and 9 overtones and combinations. See Young et al. (2008) for details.

magnitude and shape, with the observed positronmolecule annihilation resonances.

\section{B. Methanol: A case of multimode VFR}

In methyl halides, each vibrational mode produces a measurable VFR with a relative magnitude given by the $g$ factor, and there is no evidence of multimode excitations. Experiments show that this "selection rule" must be relaxed for a variety of other molecular species. As in electron-molecule collisions, positrons can be expected to excite vibrations that are nominally dipole forbidden. The nature of annihilation VFRs, as described by the theory, will be to even out fairly large variations in the capture rate as long as $\Gamma_{\nu}^{e} \gg \Gamma^{a}$. As shown in Fig. 16, there is evidence of such higher-order vibrations in the $Z_{\text {eff }}$ spectrum of methanol $\mathrm{CH}_{3} \mathrm{OH}$. This molecule is isoelectronic with $\mathrm{CH}_{3} \mathrm{~F}$. Its vibrational spectrum is also similar except for the additional $\mathrm{O}-\mathrm{H}$ stretch vibration. However, the $Z_{\text {eff }}$ spectrum of methanol is quite different than that of $\mathrm{CH}_{3} \mathrm{~F}$ and the other methyl halides. There is a significant increase in magnitude of the highand low-energy peaks in methanol relative to those of $\mathrm{CH}_{3} \mathrm{~F}$.

The positions of the $\mathrm{C}-\mathrm{H}$ stretch peak and the peaks at lower energies indicate that the binding energy in methanol is small. The fitted binding energy of $\mathrm{CH}_{3} \mathrm{~F}$ is $0.3 \mathrm{meV}$; in methanol it could be an order of magnitude larger but still remain within the experimental energy uncertainty. There is evidence of an additional peak above the $\mathrm{C}-\mathrm{H}$ stretch modes, presumably due to the $\mathrm{O}-\mathrm{H}$ stretch mode which has the energy $\omega_{\mathrm{OH}}$ $=456 \mathrm{meV}$. If this interpretation is correct, this VFR is downshifted relative to $\omega_{\mathrm{OH}}$ by an amount somewhat greater than the positron binding energy.

While one can vary the binding energy to describe better some of the enhancement in $Z_{\text {eff }}$ seen in Fig. 16, there are features in the spectrum that cannot be explained by mode-based VFRs. In particular, the higher- 
energy peak in methanol is much broader than the corresponding $\mathrm{C}-\mathrm{H}$ stretch peaks in the halomethanes and the prediction based on the IR-active modes. It is also much closer in magnitude to the low-energy peak. This discrepancy can only be resolved by considering additional resonances.

Figure 16 shows the results of two calculations using a binding energy of $2 \mathrm{meV}$. The dashed curve is the calculation which includes only the fundamental vibrations, all of which are dipole active. It falls significantly short of explaining the spectrum. The IR absorption measurements in methanol reveal a number of relatively weak overtones and combination vibrations (likely some of which are Fermi resonances) (Bertie and Zhang, 1997). Using these data allow one to estimate the elastic rates $\Gamma_{\nu}^{e}$. They are generally smaller than those of the fundamentals but still satisfy the relation $\Gamma_{\nu}^{e} \gg \Gamma^{a}$ [see Table I by Young et al. (2008)]. The result of adding these 9 two-quantum overtones and combinations to the 12 fundamentals is shown by the solid curve in Fig. 16. This prediction is clearly in better agreement with experiment. This comparison provides strong evidence that multimode vibrations can make significant contributions to the $Z_{\text {eff }}$ spectra of small molecules. There are two remaining discrepancies. One is the significantly downshifted O-H stretch peak. The second is the higher experimental $Z_{\text {eff }}$ values below $100 \mathrm{meV}$. In methanol there is a torsion mode at $\sim 40 \mathrm{meV}$. Its overtones and combinations could provide the missing spectral weight in this region, but there is at present no estimate of this effect.

\section{VFR from dipole-forbidden vibrations}

The theoretical analysis of methanol indicates that multimode vibrations can contribute significant spectral weight to an annihilation spectrum. However, there is also strong evidence that modes with very weak (or nominally zero) dipole coupling can also produce VFRs. They could, for example, arise from higher-order nondipole coupling (e.g., electric-quadrupole-active modes), but there is no simple way to assess their possible contributions. These nondipole features are exemplified by the experimental and theoretical $Z_{\text {eff }}$ spectra of ethylene shown in Fig. 17(a). This molecule has five IR-active modes and six IR-inactive modes. The shift of the C-H stretch peak indicates a binding energy of about $10 \mathrm{meV}$.

Including only IR-active ( $B_{u}$ symmetry) modes underestimates $Z_{\text {eff }}$ by a factor of 2 at the peaks located at 100 and $350 \mathrm{meV}$. This calculation, which uses the IR strengths from Bishop and Cheung (1982), completely misses contributions in the interval of energies between these modes. However, the form of Eq. (42) does not depend on the nature of positron-vibrational coupling, and all vibrations with $\Gamma_{\nu} \approx \Gamma_{\nu}^{e} \gg \Gamma^{a}$ contribute equally to $Z_{\text {eff }}$ (i.e., within the $g$ factor), as per Eq. (57). As shown in Fig. 17, including the remaining modes (i.e., those with $A_{g}$ and $B_{g}$ symmetry, corresponding to the capture

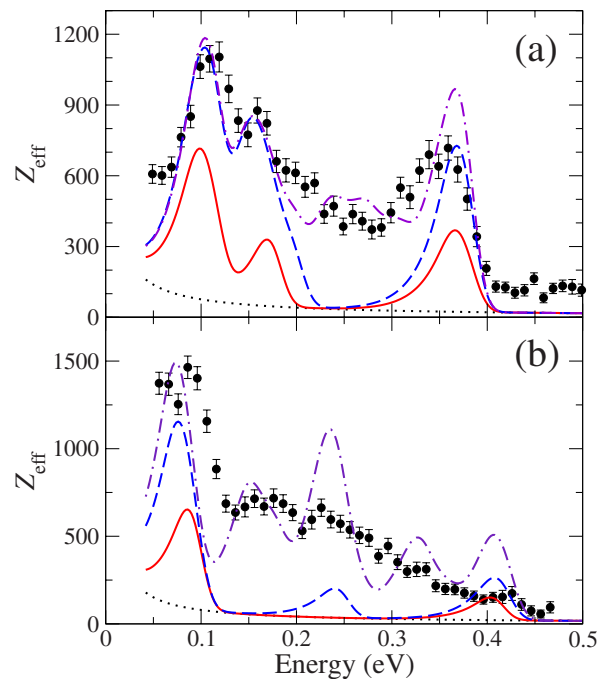

FIG. 17. (Color online) Comparison of the experimental $Z_{\text {eff }}$ (๑) with theory for (a) ethylene $\left(\mathrm{C}_{2} \mathrm{H}_{4}, \varepsilon_{b}=10 \mathrm{meV}\right)$ and (b) acetylene $\left(\mathrm{C}_{2} \mathrm{H}_{2}, \varepsilon_{b}=5 \mathrm{meV}\right)$. Dotted curves, direct annihilation $Z_{\text {eff }}^{\text {(dir) }}$; solid curves, total $Z_{\text {eff }}$ due to all IR active modes. In (a) dashed curve, total $Z_{\text {eff }}$ due to $A_{g}, B_{g}$, and $B_{u}$ modes that capture $s$-, $p$-, and $d$-wave positrons; chain curve, same with the addition of 14 IR-active overtones and combinations listed in Georges et al. (1999). In (b) long-dashed curve, all modes; chain curve, all modes with $\Sigma_{g, u}, \Pi_{g, u}$, and $\Delta_{g}$ symmetries. The contributions of all overtones and combinations are ad hoc weighted by a factor $1 / n$, where $n$ is the number of vibrational quanta.

of $s-, p$-, and $d$-wave positrons) greatly improves the agreement. The further inclusion of the 14 IR-active combination vibrations (not shown) results in $Z_{\text {eff }}$ values exceeding those observed experimentally. However, as shown in Fig. 17, if one weighs their contributions by an empirical factor $\Gamma_{\nu}^{e} / \Gamma_{\nu}=1 / n$, where $n$ is the number of vibrational quanta involved, the prediction agrees well with the experimentally measured spectrum. This analysis shows that nearly all of the spectral weight between the two largest peaks in ethylene can, at least in principle, be attributed to multimode VFR. However, there is presently only an ad hoc approach available to decide whether "borderline" vibrational capture channels sit above or below the cutoff value of the coupling strength set by $\Gamma^{a}$.

Shown in Fig. 17(b) is the spectrum of acetylene $\left(\mathrm{C}_{2} \mathrm{H}_{2}\right)$. Its binding energy is too small to measure directly, and hence it is obtained by fitting to the spectrum. As in the case of ethylene, the theoretical $Z_{\text {eff }}$, which includes only IR-active fundamentals, does not agree with the measured spectrum. Adding all of the other modes provides little improvement. It is only after adding the overtones and combinations that the calculated $Z_{\text {eff }}$ spectrum matches the magnitude of that observed. Still, the peaks in the theoretical $Z_{\text {eff }}$ spectrum are much more prominent. A similar analysis has been performed for ammonia (Gribakin, 2010). It also highlights the role of overtones and combinations, and suggests that rotational broadening of VFR and rotational Feshbach reso- 


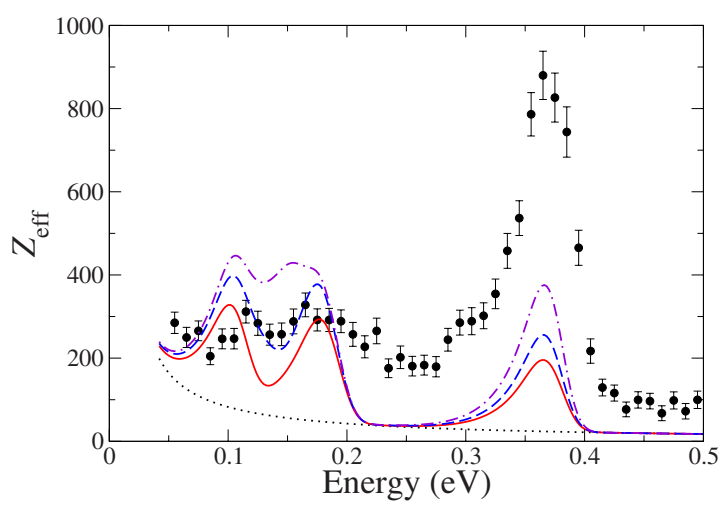

FIG. 18. (Color online) Experimental $Z_{\text {eff }}$ spectrum (๑) and theory for ethane $\left(\mathrm{C}_{2} \mathrm{H}_{6}\right)$. The calculations use $\varepsilon_{b}=1 \mathrm{meV}$ and include: dotted curve, direct $Z_{\text {eff }}$; solid curve, same with VFR of IR-active modes; dashed curve, same with the addition of $A_{1 g}$ modes; chain curve, same with the addition of $E_{g}$ modes.

nances may be required to explain the $Z_{\text {eff }}$ spectrum in this small molecule.

The modeling discussed here indicates that, to explain the $Z_{\text {eff }}$ spectra of molecules such as $\mathrm{C}_{2} \mathrm{H}_{4}$ and $\mathrm{C}_{2} \mathrm{H}_{2}$, one needs to include the VFR of IR-inactive modes, overtones, and combinations. However, determining $\Gamma_{\nu}^{e}$ and $\Gamma_{\nu}$ for these resonances requires calculations of the full vibrational dynamics, and so at present the theory is incomplete.

\section{Effect of molecular size on the magnitudes of VFR}

As discussed in the next section, larger molecules exhibit annihilation VFRs that cannot be explained by positron coupling to fundamentals or combination and overtone vibrations. In these molecules, an additional enhancement mechanism appears to be operative that causes the magnitudes of the fundamental resonances to grow rapidly with molecule size. The smallest molecule to show evidence of such enhanced VFR is ethane. As shown in Fig. 18, the $Z_{\text {eff }}$ spectrum for this molecule has a distinctly different spectral shape than that of the other molecules discussed in this chapter. The highenergy C-H stretch peak is three times larger than the low-energy $\mathrm{C}-\mathrm{H}$ bend peaks, which is inconsistent with the simple $g$ scaling of VFR magnitudes. The calculated $Z_{\text {eff }}$ for ethane, shown in Fig. 18, indicates that the VFRs of IR-active and dipole-forbidden modes, populated by the positron $s-, p-$, and $d$-wave capture, can explain the spectral weight at lower energies. However, they do not account for the magnitude of the $\mathrm{C}-\mathrm{H}$ stretch peak, nor for the magnitude of $Z_{\text {eff }}$ between the low- and highenergy peaks, which may be due to overtones and combinations.

Similar analyses for propane and cyclopropane (see Sec. V.F) show that these molecules exhibit even stronger enhancements of the high- and low-energy peaks, while the "background" of multimode excitations between these resonances does not appear to be similarly enhanced. However, molecular size alone does not ap-

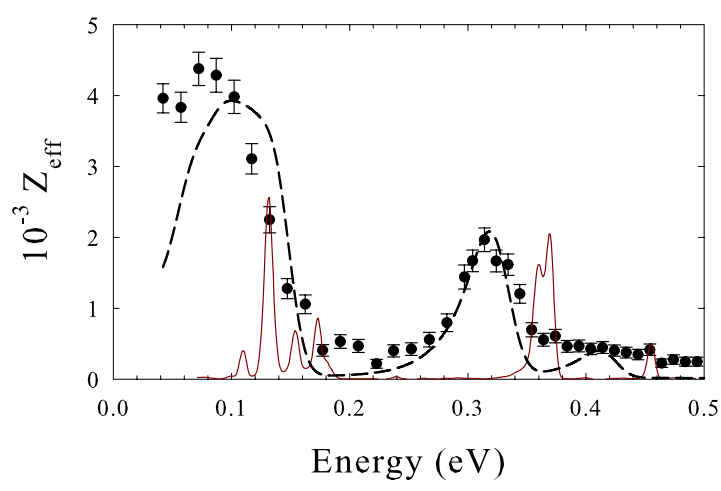

FIG. 19. (Color online) Comparison of experimental $Z_{\text {eff }}(\bullet)$, IR absorption spectrum (solid curve), and theoretical $Z_{\text {eff }}$ (dashed curve) for ethanol $\left(\mathrm{C}_{2} \mathrm{H}_{5} \mathrm{OH}\right)$. The calculation [cf. Eq. (42)] uses $\varepsilon_{b}=45 \mathrm{meV}$ and the mode frequencies and transition dipole amplitudes from Shaw et al. (1990).

pear to be an accurate predictor of the scaling of $Z_{\text {eff }}$ magnitudes. As shown in Fig. 19, the analysis for ethanol $\left(\mathrm{C}_{2} \mathrm{H}_{5} \mathrm{OH}\right.$; one more atom than ethane) indicates that there is no evidence of such an enhancement for this molecule. Here the agreement with experimental $Z_{\text {eff }}$ of the simple IR-active-mode VFR theory without overtones or combinations is remarkably good.

In summary, the theoretical framework of Gribakin and Lee (2006a) allows us to make a clearer distinction between those molecules that exhibit "small molecule" behavior and those that exhibit "large molecule" behavior (i.e., where $Z_{\text {eff }}$ values can be orders of magnitude larger than those predicted by the mode VFR theory). However, the physics that is responsible for this threshold is still poorly understood.

\section{E. Nonresonant annihilation in small molecules}

Many molecules do not exhibit resonant annihilation but display instead relatively smooth featureless $Z_{\text {eff }}$ spectra. To better understand these molecules, recall that the minimum requirements to observe annihilationmediated VFR are the existence of a positron-molecule bound state and a vibrational mode that couples to the positron.

The existence of weak binding with $\varepsilon_{b}=\kappa^{2} / 2$ is linked to a large positive value of the positron-molecule scattering length $\kappa^{-1}$ (Sec. II.D). The magnitudes of the resonances are then determined by $g=\kappa / k$. If $\kappa$ is negative, the bound state is replaced by a virtual state in the continuum, and VFR are absent. In both cases, however, one expects a nonresonant background due to direct annihilation, proportional to $\left(k^{2}+\kappa^{2}\right)^{-1}$ [cf. Eq. (29)].

Small nonpolar or weakly polar molecules are far less likely to bind positrons, and so it is not surprising that many of these molecules lack VFRs. One such molecule is $\mathrm{CO}_{2}$. It has a relatively flat spectrum with $Z_{\text {eff }} \approx 35$ above $150 \mathrm{meV}$ (Young and Surko, 2008c), and the thermal $Z_{\text {eff }}$ of 54.7 (Wright et al., 1985). Since this molecule has 22 electrons, these values are not far from the uncorrelated electron gas prediction. Using a vibrational 


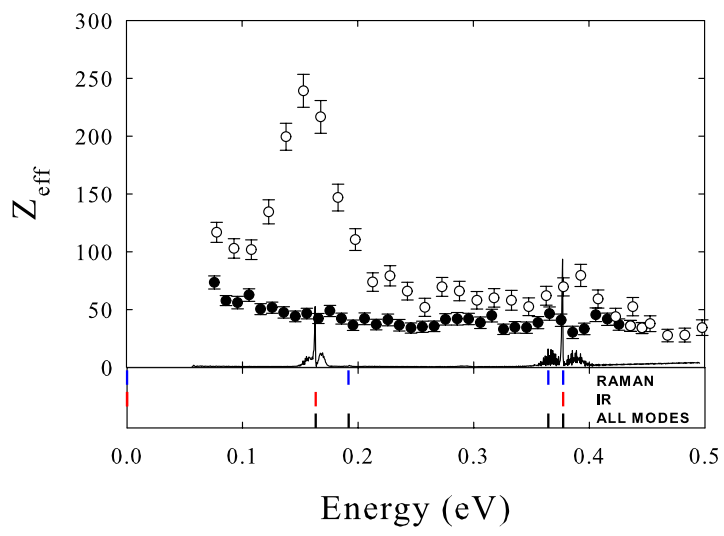

FIG. 20. (Color online) Measured $Z_{\text {eff }}$ spectra for $\mathrm{CH}_{4}(-)$ and $\mathrm{CH}_{3} \mathrm{~F}(\mathrm{O})$ and IR absorption spectrum of methane (solid curve).

close coupling formalism, Gianturco and Mukherjee (1999) predicted a resonance-free spectrum with a nearly constant $Z_{\text {eff }} \approx 50$, which is in reasonably good agreement with the measurements.

The spectrum of methane $\mathrm{CH}_{4}$, shown in Fig. 20, is also relatively featureless and similar in both magnitude and energy dependence to the $Z_{\text {eff }}$ for $\mathrm{CF}_{4}$ (not shown) (Barnes et al., 2003). This is consistent with these molecules not supporting the positron bound states. It refutes an earlier conjecture (Gribakin, 2000; Iwata et al., 2000), which was based on the analysis of roomtemperature $Z_{\text {eff }}$ for methane and its fluorosubstitutes. However, the difference between molecules close to the border between VFR-active and VFR-inactive species can be stark. Figure 20 shows a comparison of $\mathrm{CH}_{4}$ and $\mathrm{CH}_{3} \mathrm{~F}$. The analysis presented in Fig. 15 indicates that $\mathrm{CH}_{3} \mathrm{~F}$ has a very small binding energy $(\sim 0.3 \mathrm{meV})$. Yet this produces a distinct resonance at $150 \mathrm{meV}$, and the spectral weight in the vicinity of the $\mathrm{C}-\mathrm{H}$ stretch mode is nearly doubled.

As shown in Fig. 21, the water molecule also lacks distinct VFR (Young and Surko, 2008c). Its $Z_{\text {eff }}$ rises smoothly with decreasing energy, reaching a value of 319 for thermal positrons at $300 \mathrm{~K}$ (Iwata et al., 1995). The spectrum is well represented by the expression for direct annihilation [Eq. (29)]. ${ }^{20}$ The fitting parameter $\kappa$ is consistent with a virtual state at $\sim 1 \mathrm{meV}$. A constant $Z_{\text {eff }}$ offset of 20 was also included in this fit [cf. the first term in Eq. (28)].

The $Z_{\text {eff }}$ data for these small polyatomics can be compared with the direct annihilation calculations of Gianturco et al. (2001). In that work, the positron-molecule interaction is described by a local correlationpolarization potential, and $Z_{\text {eff }}$ includes the electronpositron contact density enhancement factor (Boronski and Nieminen, 1986). The calculations predict a steady rise in $Z_{\text {eff }}$ with decreasing positron energy below $0.5-1 \mathrm{eV}$. The calculated annihilation rates for $\mathrm{CH}_{4}$,

\footnotetext{
${ }^{20}$ In principle, Eq. (29) should be modified to account for the permanent dipole moment of this molecule.
}

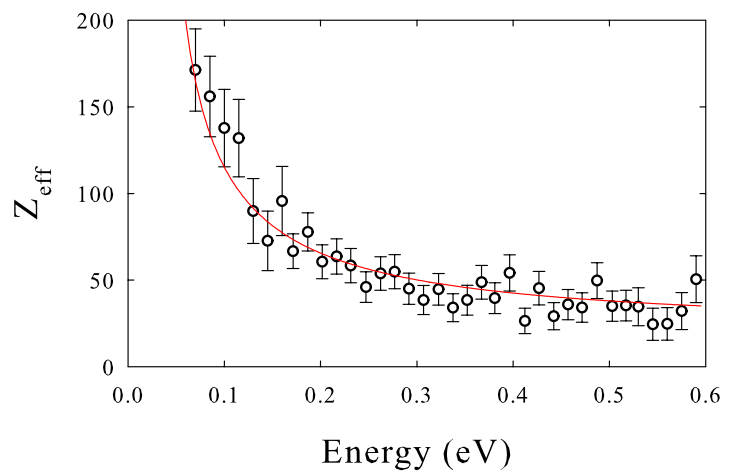

FIG. 21. (Color online) Experimental $Z_{\text {eff }}$ spectrum (O) for water, and a fit based on the direct annihilation model: solid curve, $Z_{\text {eff }}$ from Eq. (29) with $\kappa^{2} / 2=0.3 \mathrm{meV}$ plus a constant offset of 20 .

$\mathrm{NH}_{3}$, and $\mathrm{H}_{2} \mathrm{O}$ (including those at thermal positron energies) are about a factor of 2 lower than the experimental values, while for $\mathrm{CF}_{4}$, the calculated $Z_{\text {eff }}$ is about two times larger. With the exception of $\mathrm{NH}_{3}$ (Gribakin, 2010), the calculations confirm that VFR are not necessary to explain the $Z_{\text {eff }}$ spectra in these molecules.

Some molecules exhibit other spectral features. One example is the sawtoothlike oscillation centered at $\sim 380 \mathrm{meV}$ in $\mathrm{CH}_{4}$ (Fig. 20). While the IR spectrum indicates strong absorption at this energy, the magnitude and shape of this feature in $Z_{\text {eff }}$ are not consistent with the VFR observed in other molecules. Such sawtooth features are also observed in the $Z_{\text {eff }}$ spectra of $\mathrm{CO}_{2}$ and $\mathrm{H}_{2} \mathrm{O}$ (Young and Surko, 2008c).

The origin of these features is at present unclear. Nishimura and Gianturco (2005a) predicted that water should have fairly strong vibrational excitation cross sections, with sharp onsets. Channel coupling could result in additional structure in the $Z_{\text {eff }}$ spectrum near the vibrational excitation thresholds (Young and Surko, 2008c). Alternatively, Young and Surko (2008c) suggested that these features could represent interference between direct and resonant annihilation. However, the latter is not compatible with the evidence that $\mathrm{CO}_{2}$, $\mathrm{H}_{2} \mathrm{O}$, and $\mathrm{CH}_{4}$ do not bind positrons.

Other non-VFR-type features are either predicted or allowed by theory but have yet to be observed. One example is a shape resonance which could occur if the positron became temporarily trapped inside a positiveenergy potential barrier. However, low-energy positron scattering and annihilation is usually dominated by the $s$-wave component of the incident positron wave function, which has no centrifugal barrier. Further, the atomic cores are repulsive, so a shape resonance is unlikely. Exceptions in other systems include the prediction of a $p$-wave positron shape resonance in the $\mathrm{Mg}$ atom (Mitroy and Bromley, 2007; Mitroy et al., 2008) and cage-state shape resonances in cubane $\left(\mathrm{C}_{8} \mathrm{H}_{8}\right), \mathrm{C}_{20}$, and $\mathrm{C}_{60}$ (Gianturco and Lucchese, 1999; Gianturco et al., 2005; Carey et al., 2008). Finally, Nishimura and Gianturco (2003) hypothesized that the presence of a virtual state, by itself, can lead to a long-lived intermediate 
state (e.g., following a vibrational deexcitation collision), and this is expected to produce a broad spectral feature. Experimental investigation of these predictions is warranted.

\section{F. Small molecule summary}

The theory of Gribakin and Lee (2006a) (Sec. II.F) provides a remarkably useful framework for understanding resonant annihilation in small molecules. The relative contribution of each VFR is a competition between resonant elastic scattering and annihilation. As long as the coupling to the positron continuum is strong enough, the magnitude of each resonance is proportional to $g=\sqrt{\varepsilon_{b} / \varepsilon}$, and the annihilation spectrum is a sum of distinct resonances.

In the case of the methyl halides and ethanol, the conditions for the simple $g$ scaling were confirmed by direct calculation of the capture rates using the Born-dipoletype approximation (Sec. II.F). For methyl halides, the binding energies were extracted from the measurements on the protonated species. They were then used to make a prediction of $Z_{\text {eff }}$ for the deuterated species, thus providing a stringent test of the theory. Agreement between experiment and theory is excellent.

In other cases, however, the VFR of the IR-active modes are insufficient to explain the observed annihilation spectra. In methanol, it is necessary to include dipole-active multimode vibrations; and in ethylene and acetylene, one must also include IR-inactive modes and $n$-quantum overtones and combinations to match the experimentally measured $Z_{\text {eff. }}$ In these cases, the VFR of overtone and combination vibrations had to be adjusted arbitrarily (i.e., by factors $1 / n$ ) to match the experimental $Z_{\text {eff. }}$ Thus, while the theory of Sec. II.F provides useful insights into the vibrations that are likely involved, it lacks quantitative predictive accuracy for many molecules.

This theory has also helped to elucidate the boundary separating species that have enhanced $Z_{\text {eff }}$ (i.e., beyond that predicted by single resonances) from those that do not. The factors responsible for this transition appear to be molecular size and the density of vibrational states near a resonance (Sec. II.G). This enhancement is likely due to IVR. There is some evidence that it is already operative in molecules as small as ethane and is dominant in propane.

\section{IVR-ENHANCED RESONANT ANNIHILATION IN LARGER MOLECULES}

\section{A. Overview}

As indicated in Table I, annihilation rates for large molecules grow rapidly with molecular size. Similar to the case of small molecules, these large rates are understood to be the result of positron attachment via VFR. As shown in Fig. 1 for butane, the $Z_{\text {eff }}$ spectra have distinct peaks, the positions of which are strongly correlated with those of the IR peaks but are shifted down-

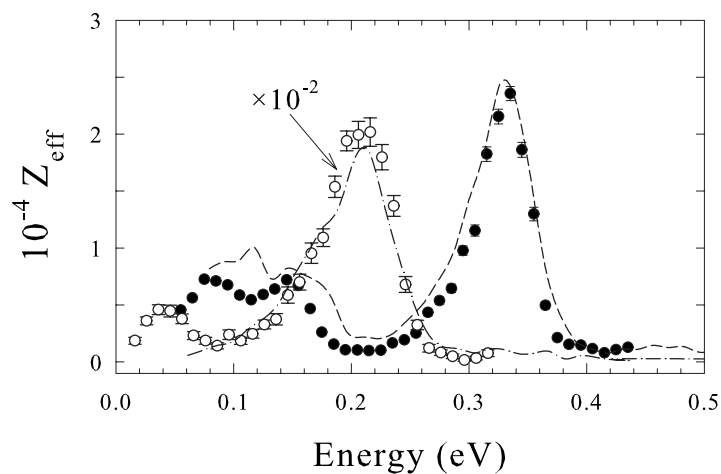

FIG. 22. $Z_{\text {eff }}$ spectra of hydrogenated butane (solid circles) (Barnes et al., 2003) and nonane (open circles) (Young and Surko, 2008b) compared with (the appropriately scaled) fully deuterated analogs (dashed and chain curves) (Barnes et al., 2003). Mode energies for the deuterated species have been scaled by the appropriate reduced mass factor to match those in the hydrogenated compounds, while the positron binding energy was assumed to be independent of deuteration. The magnitudes of $Z_{\text {eff }}$ have been scaled by the appropriate $g$ factor at each incident positron energy.

ward by the binding energy. As was the typical case for small molecules, the VFR in larger molecules also occur predominantly at energies corresponding to those of the fundamental vibrations. The low-energy plateau in the alkane spectra (e.g., below $140 \mathrm{meV}$ in Fig. 1) is due to $\mathrm{C}-\mathrm{H}$ bend modes and $\mathrm{C}-\mathrm{C}$ modes, while the high-energy peaks are due to $\mathrm{C}-\mathrm{H}$ stretch modes. In the case of large molecules, however, the $Z_{\text {eff }}$ values in these peaks are enhanced by orders of magnitude above those predicted for VFR in small molecules.

To validate the identification of the resonant peaks with the vibrational modes, shown in Fig. 22 is the spectrum of butane and nonane and the corresponding fully deuterated compounds (Barnes et al., 2003; Young and Surko, 2008b). When the spectra of the deuterated compounds are corrected for the change in vibrational mode frequencies, the deuterated and hydrogenated data are in good agreement.

As discussed in Sec. II, a plausible explanation for the large magnitudes of $Z_{\text {eff }}$ in large molecules is that, by the excitation of a vibrational fundamental, a large number of otherwise inaccessible multimode VFR (so-called dark states) contribute to the annihilation, mediated by the process of intramolecular vibrational energy redistribution (IVR) (Gribakin, 2000; Gribakin and Gill, 2004). In this paradigm, the incident positron is first captured into a mode-based doorway state (e.g., involving infrared active modes) that then couples to a bath of quasidegenerate multimode dark states. This increases the multiplicity of the final capture states and causes an approximately proportionate enhancement in the resonant annihilation rate. As discussed below, it appears that the fundamental vibrations act as doorways in large molecules. This in turn leads to the excitation of some, but not all of the nearby multimode states in the IVR process, which results in enhanced annihilation. A simi- 


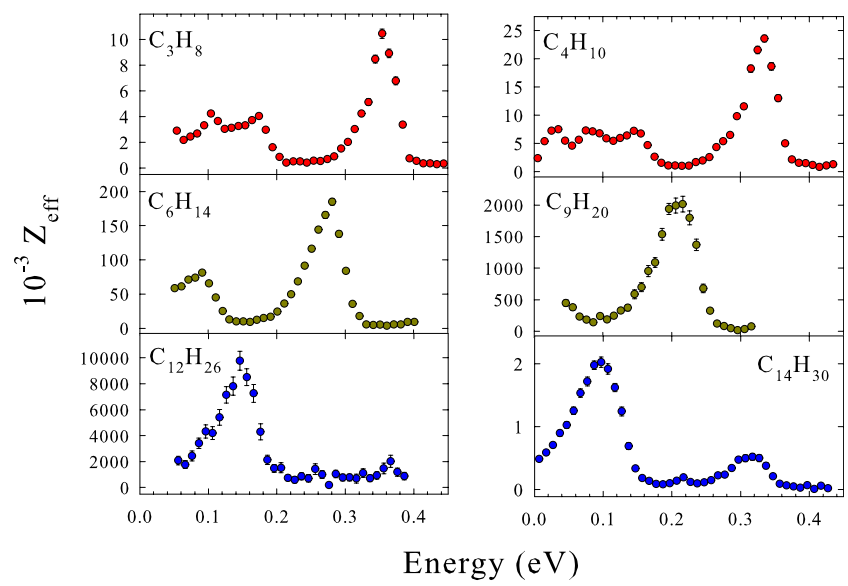

FIG. 23. (Color online) $Z_{\text {eff }}$ spectra as a function of incident positron energy for a variety of alkane molecules, showing the systematic shift of the spectra to lower positron energy as molecular size is increased. Values of $Z_{\text {eff }}$ are absolute except for $\mathrm{C}_{14} \mathrm{H}_{30}$, which is in arbitrary units due to difficulties measuring its vapor pressure.

lar coupling to dark states occurs in molecular photoabsorption (Stewart and McDonald, 1983).

This doorway-state model provides a common thread in the discussion of annihilation for large molecules. A particularly important link in this model is the connection between the observed rapid rise in the resonant annihilation rate with molecular size and IVR. A number of experiments and analyses clarify and, in some cases quantify, this physical picture.

\section{B. The alkane molecule paradigm}

Alkane molecules have been studied more extensively than any other molecular species (cf. Figs. 1 and 22). Figure 23 shows six examples. These spectra resemble closely the spectra of the fundamental vibrations, albeit downshifted by the positron-molecule binding energy. The binding energies increase by $20-25 \mathrm{meV}$ for each carbon-based monomer added to the alkane chain. Thus, the binding energy for propane is $10 \mathrm{meV}$, while for nonane it is $145 \mathrm{meV}$. The magnitudes of the resonant annihilation peaks grow rapidly with molecular size, indicating that IVR, albeit likely incomplete, is operative.

The theory of resonant annihilation for small molecules (Sec. II.F) prescribes a restricted role for binding energy in determining $Z_{\text {eff }}$ spectra, namely,

$$
Z_{\text {eff }} \propto b_{\nu} g(\varepsilon)=b_{\nu} \sqrt{\varepsilon_{b} / \varepsilon},
$$

where $b_{\nu}$ is the multiplicity of the excited resonant capture states. The vibrational coupling appears only via the positron capture rate $\Gamma^{e}$, which usually cancels the total rate $\Gamma$ for all but very weak resonances.

As shown in Fig. 24, the $Z_{\text {eff }}$ spectra for alkanes larger than ethane are self-similar when they are first scaled by the factor $g(\varepsilon)$, then the resulting magnitudes of $Z_{\text {eff }} / g(\varepsilon)$ are normalized at the $\mathrm{C}-\mathrm{H}$ stretch peaks, and,

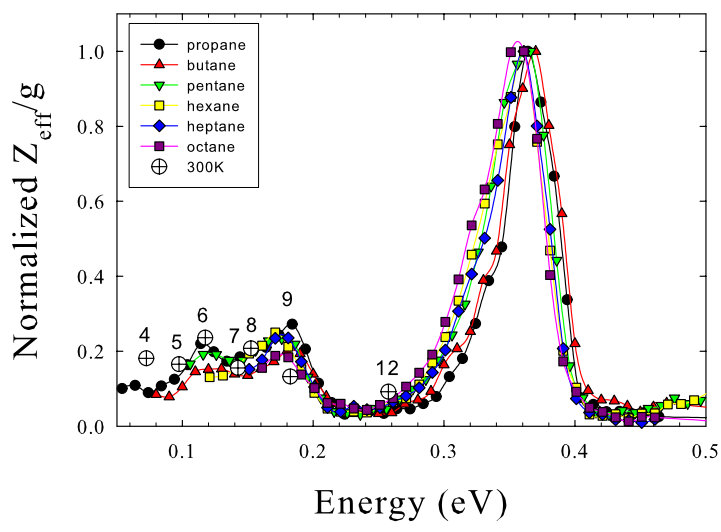

FIG. 24. (Color online) Normalized and energy-shifted $Z_{\text {eff }} / g$ spectra for alkanes with $n=3-8$ carbons. For comparison, room-temperature $Z_{\text {eff }} / g$ data for alkanes are also shown (circles with plus sign) at energies $\varepsilon_{b}+\varepsilon_{T}$, where $\varepsilon_{T}=\frac{3}{2} k_{B} T$ $=37.5 \mathrm{meV}$. Each room-temperature datum is labeled by the number of carbons $n$ in the molecule. See text for details.

finally, the spectra are shifted upward by their binding energies (Young and Surko, 2008b). A surprising result from this analysis, not presently understood, is that the relative magnitudes of the high-energy and lower-energy peaks in the alkanes remain the same over a factor of 3 in molecular size and a factor of $10^{3}$ in the magnitude of $Z_{\text {eff. }}$ The resulting self-similar spectra shift downward with increasing molecular size as the positron binding energy increases. As discussed in Sec. VII, this has the consequence that the $Z_{\text {eff }}$ spectrum, measured with thermal positrons, can be assigned to the vibrational modes populated through the corresponding VFRs in the thermal energy range. This connects in a quantitative way the energy-resolved measurements and thermal measurements of $Z_{\text {eff. }}$.

As the size of the alkane is increased beyond 12 carbons (dodecane), a new feature appears in the $Z_{\text {eff }}$ spectrum at an energy close to that of the $\mathrm{C}-\mathrm{H}$ stretch peak. As shown in Fig. 25, the magnitude of this peak grows as the alkane size increases, and it shifts downward in energy, just as the original $\mathrm{C}-\mathrm{H}$ stretch peak. In hexadecane (the 16-carbon alkane), the $\mathrm{C}-\mathrm{H}$ peak occurs at a mere $55 \mathrm{meV}$ incident positron energy, corresponding to a binding energy of $310 \mathrm{meV}$.

This new resonance is attributed to a second positron bound state (i.e., the first positronically excited state) on the molecule. It is populated by a C-H stretch peak VFR in a manner similar to the larger ground-state peak that occurs at smaller incident positron energy (Barnes et al., 2006). The small peak at $365 \mathrm{meV}$ in dodecane is identified as the C-H stretch peak of the first, positronically excited bound state with a binding energy of a few millielectron volts, while the larger peak at $150 \mathrm{meV}$ is due to the positron in its ground state. The positions of these peaks are in good agreement with a model calculation described in Sec. II.H.2 (cf. Fig. 9).

An important feature of these positronically excited resonances is their magnitude relative to the corresponding ground-state resonance in the same molecule. 


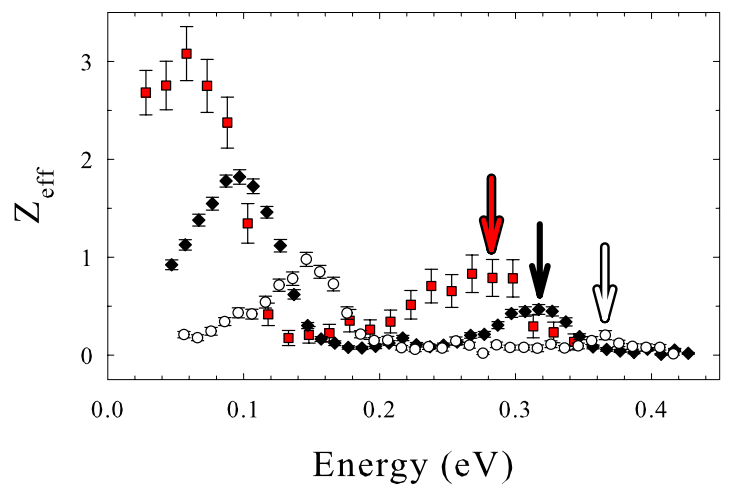

FIG. 25. (Color online) $Z_{\text {eff }}$ spectra for $(\bigcirc)$ dodecane, (diamonds) tetradecane, and (squares) hexadecane. The vertical arrows indicate the positions of the $\mathrm{C}-\mathrm{H}$ stretch mode VFR peaks for the second bound state (i.e., the first positronically excited state) in each molecule. The spectra for tetradecane and hexadecane have been normalized arbitrarily since their vapor pressures were too low to measure reliably. The large peaks at lower energy are the $\mathrm{C}$-H stretch mode VFR for the first bound states (the positronic ground states).

For small molecules, it is expected that the contribution of a resonance will be proportional to $g=\sqrt{\varepsilon_{b} / \varepsilon}$. For both tetradecane and hexadecane, the ratios of the magnitudes of the first and second bound-state C-H stretch resonances are equal to the ratios of the $g$ factors for these resonances. In particular, since the positron overlap density is proportional to $\sqrt{\varepsilon_{b}}$ (Sec. II.H.2), it is expected to be smaller for the second bound state, due to the smaller binding energy. This scaling of $Z_{\text {eff }}$ for the positronically excited states and the scaling with $g$ of the spectra shown in Fig. 24 both demonstrate the important role of the factor $g$ in determining the magnitudes of annihilation peaks in large molecules.

\section{Dependence of $Z_{\text {eff }}$ on molecular size}

As shown in Fig. 26, positron-molecule annihilation has been studied for a variety of chemical species. While the vibrational modes and energy levels in these molecules differ in various ways, all of the hydrocarbons studied contain strong $\mathrm{C}-\mathrm{H}$ stretch vibrational modes that result in prominent annihilation resonances. Consequently, they provide a convenient benchmark to determine positron-molecule binding energies and the relative magnitudes of the annihilation rates.

Relatively small changes in molecular structure can have significant effects on both the positron binding energies and the overall annihilation rates. For example, the $\mathrm{C}-\mathrm{H}$ stretch peak in 1-chlorohexane is shifted downward relative to that of hexane by nearly $100 \mathrm{meV}$, while the magnitude of the $\mathrm{C}-\mathrm{H}$ stretch resonance is increased by nearly a factor of 3 (Young and Surko, 2008b).

Based upon the alkane data, one might conclude that binding energy and the magnitudes of the annihilation resonances are strongly correlated (Young and Surko, 2008b). However, as shown in Fig. 26, this hypothesis is not borne out by the data. With the exception of the

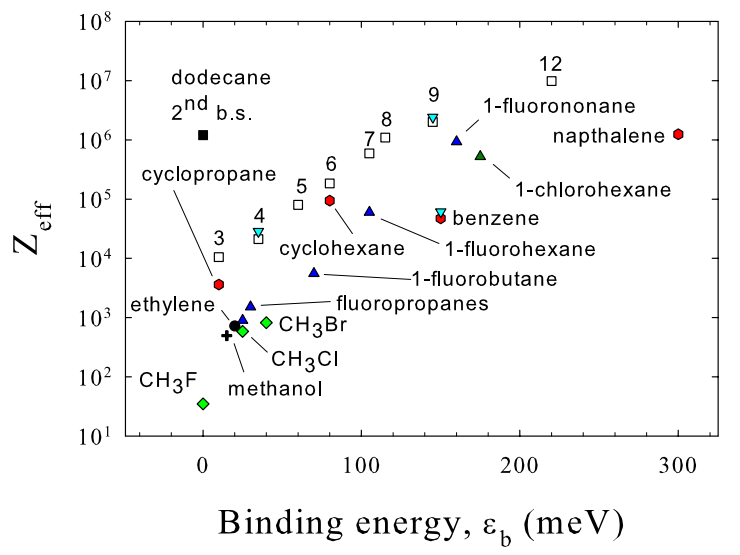

FIG. 26. (Color online) $Z_{\text {eff }}$ at the C-H stretch peak $v s$ binding energy $\varepsilon_{b}$ for alkanes, $\mathrm{C}_{n} \mathrm{H}_{2 n+2}(\square)$, with the number of carbons $n$ indicated; rings (hexagons); halomethanes $(\diamond)$; ethylene $(\circlearrowleft)$; methanol $(+)$; 1-chlorohexane $(\triangle)$; fluoroalkanes $(\triangle)$; and deuterated species $(\nabla)$.

alkanes, the binding energies and the magnitudes of the $\mathrm{C}-\mathrm{H}$ stretch resonances appear to be only weakly correlated. For example, the $Z_{\text {eff }}$ values for 1-chlorohexane, benzene, and naphthalene are an order of magnitude or more smaller than those for alkanes with similar binding energies.

This lack of correlation of $Z_{\text {eff }}$ with binding energy motivated further analysis of the $Z_{\text {eff }}$ magnitudes. Shown in Fig. 27 and listed in Table V are data for $Z_{\text {eff }}$ the $\mathrm{C}-\mathrm{H}$ stretch peak, normalized by $g$, as a function of the number of atoms $N$. With the exception of the partially fluorinated compounds, which will be discussed below, the magnitudes of the annihilation rates for the relatively wide variety of molecules studied lie close to a universal curve. 1-chlorohexane and benzene are no longer outliers to the extent they were in Fig. 26. The empirical scaling of $Z_{\text {eff }}$ with $N$ is found to be (Young and Surko, 2007, 2008b),

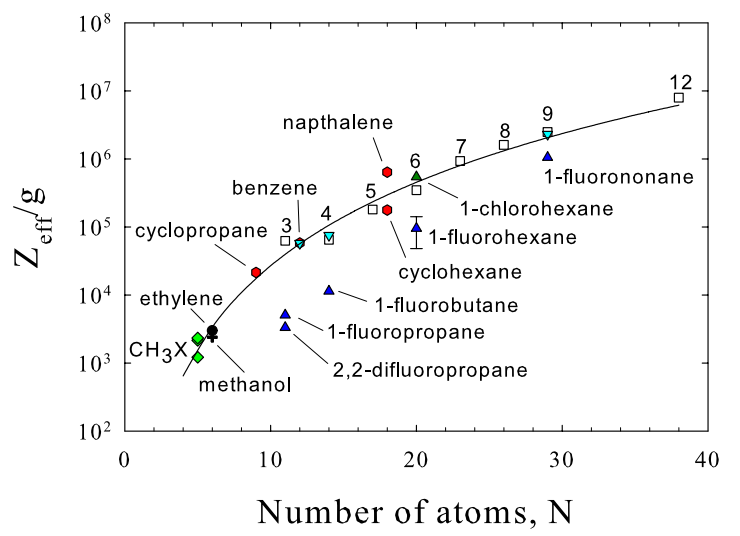

FIG. 27. (Color online) $Z_{\text {eff }}$ at the C-H stretch peak, normalized by the factor $g=\sqrt{\varepsilon_{b} / \varepsilon}$, vs the number of atoms in the molecule. The symbols are as in Fig. 26. The solid line is the fit given by Eq. (59). The error bars on 1-fluorohexane indicate the unusually large uncertainty for this datum. 
TABLE V. Parameters for annihilation on various large molecules: the number of atoms $N$, positron binding energies, $Z_{\text {eff }}$ at the $\mathrm{C}-\mathrm{H}$ stretch mode and for thermal distributions of positrons at $300 \mathrm{~K}$,

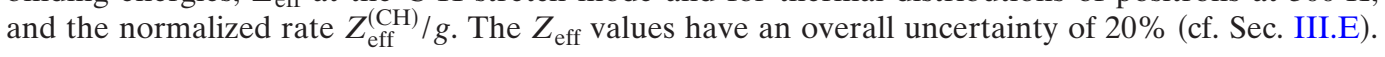

\begin{tabular}{|c|c|c|c|c|c|}
\hline Species & $N$ & $\begin{array}{c}\varepsilon_{b}{ }^{\mathrm{a}} \\
(\mathrm{meV})\end{array}$ & $Z_{\text {eff }}^{(\mathrm{CH}) \mathrm{b}}$ & $Z_{\mathrm{eff}}^{\text {th c }}$ & $Z_{\mathrm{eff}}^{(\mathrm{CH})} / g$ \\
\hline \multicolumn{6}{|c|}{ Alkanes } \\
\hline Methane & 5 & $<0$ & & 142 & \\
\hline Ethane & 8 & $>0$ & 900 & 660 & \\
\hline Propane & 11 & 10 & 10500 & 3500 & 63000 \\
\hline Butane & 14 & 35 & 21000 & 11300 & 65000 \\
\hline Pentane & 17 & 60 & 80000 & 37800 & 180000 \\
\hline Hexane & 20 & 80 & 184000 & 120000 & 350000 \\
\hline Heptane & 23 & 105 & 590000 & 242000 & 930000 \\
\hline Octane & 26 & 115 & 1090000 & 585000 & 1610000 \\
\hline Nonane & 29 & 145 & $2000000^{\mathrm{d}}$ & 643000 & 2500000 \\
\hline Dodecane & 38 & 220 & 9800000 & 1780000 & 8000000 \\
\hline 2nd bound state & 38 & $>0$ & 1200000 & & \\
\hline Tetradecane & 44 & 260 & $11 x^{\mathrm{e}}$ & & $6.8 x^{\mathrm{e}}$ \\
\hline 2nd bound state & 44 & 50 & $2.8 x^{\mathrm{e}}$ & & $7.0 x^{\mathrm{e}}$ \\
\hline Hexadecane & 50 & 310 & $15 y^{\mathrm{e}}$ & 2230000 & $6.4 y^{\mathrm{e}}$ \\
\hline 2nd bound state & 50 & 100 & $4.0 y^{\mathrm{e}}$ & & $6.5 y^{\mathrm{e}}$ \\
\hline \multicolumn{6}{|c|}{ Alkane isomers } \\
\hline Isopentane & 17 & 60 & 80000 & 50500 & 180000 \\
\hline \multicolumn{6}{|c|}{ Rings } \\
\hline Cyclopropane & 9 & 10 & 3600 & & 21500 \\
\hline Cyclohexane & 18 & 80 & 94000 & 20000 & 180000 \\
\hline Benzene & 12 & 150 & 47000 & 15000 & 58000 \\
\hline Naphthalene & 18 & 300 & 1240000 & 494000 & 640000 \\
\hline \multicolumn{6}{|c|}{ Haloalkanes } \\
\hline 1-fluoropropane & 11 & 30 & 1520 & & 5100 \\
\hline 2,2-difluoropropane & 11 & 25 & 900 & 8130 & 3300 \\
\hline 1-fluorobutane & 14 & 70 & 5600 & & 11500 \\
\hline 1-fluorohexane & 20 & 105 & $(60 \pm 30) \times 10^{3}$ & 269000 & $(94 \pm 47) \times 10^{3}$ \\
\hline 1-chlorohexane & 20 & 175 & 520000 & & 540000 \\
\hline 1-fluorononane & 29 & 160 & 930000 & & 1050000 \\
\hline \multicolumn{6}{|c|}{ Deuterated } \\
\hline$d$-benzene & 12 & 150 & 61000 & 36900 & 57500 \\
\hline$d$-butane & 14 & 35 & 28500 & & 75000 \\
\hline$d$-nonane & 29 & 145 & 2400000 & 641000 & 2300000 \\
\hline$d$-naphthalene & 18 & $\sim 300$ & & & \\
\hline
\end{tabular}

${ }^{a}$ Determined from the energy shift of the C-H peak in $Z_{\text {eff }}$.

${ }^{\mathrm{b}}$ At the C-H stretch peak (Gilbert et al., 2002; Barnes et al., 2003, 2006; Young and Surko, 2007, 2008b).

${ }^{c}$ For thermal positrons at $300 \mathrm{~K}$ (Heyland et al., 1982; Wright et al., 1983; Iwata et al., 1994, 1995; Iwata, 1997; Iwata, Greaves, and Surko, 1997).

${ }^{\mathrm{d}} Z_{\mathrm{eff}}^{(\mathrm{CH})}$ for nonane is from Young and Surko (2008b) as opposed to Barnes et al. (2003), as the latter had a narrower positron energy distribution for this molecule.

${ }^{\mathrm{e}}$ Absolute $Z_{\text {eff }}$ could not be determined, so the values are multiplied by arbitrary factors $x$ and $y$. 


$$
Z_{\text {eff }} / g=2.3 N^{q},
$$

with $q=4.1 \pm 0.1$. This scaling likely reflects the dependence of the total number of accessible positronmolecule vibrational states (i.e., including dark states) on the number of vibrational degrees of freedom.

It is not surprising that $Z_{\text {eff }}$ depends linearly on $g$ $=\kappa / k$, so long as the weak binding picture (i.e., $\kappa$ $\ll 1$ a.u.) is valid. Furthermore, the $1 / k$ factor arises from the normalization of the incident positron wave function. The surprising aspect of this $g$ scaling is that it appears to incorporate the only dependence of $Z_{\text {eff }}$ on $\varepsilon_{b}$. While the overall vibrational density of states is correlated with the number of atoms $N$, it can certainly change without $N$ changing (e.g., by chemical substitution). Further, small-molecule theory assumes that the lifetime of a vibrationally excited positron-molecule complex is short compared to the annihilation time. However, if the states accessible by IVR are especially long lived, the magnitudes of the resonances might then depend upon the capture rate rather than $g$. The annihilation rate would then be expected to saturate, growing only linearly with molecular size. The fact that the scaling of Eq. (59) remains valid for the largest molecules studied to date is evidence that this saturation limit has not yet been reached.

\section{Toward a model of annihilation in large molecules}

Our present theoretical understanding of the annihilation spectra of large molecules is incomplete. As discussed in Sec. II, it is not possible to explain the large values of $Z_{\text {eff }}$ that are observed using only VFR involving the vibrational fundamentals. This difficulty could possibly be overcome by considering mode-based resonances as doorways for positron capture in complex multimode VFRs. However, estimates assuming complete IVR show that $Z_{\text {eff }}$ would increase much faster with molecular size than is observed (Sec. II.G.1). Further, when all combination and overtone vibrations are assumed to couple to the positron continuum, the resulting spectrum is predicted to be featureless, bearing little resemblance to the experimentally measured spectra. Nevertheless, the presently available theoretical models do provide a useful framework with which to interpret some of the experimental results. In the following, additional experiments and analysis are discussed that elucidate details of VFR-mediated annihilation in large molecules and place constraints on viable theoretical models of this process.

\section{E. Inelastic autodetachment}

Inelastic escape channels are a potentially important mechanism that can limit the magnitudes of resonant annihilation peaks (Sec. II.G). They can occur when a resonantly captured positron is released from the molecule by the deexcitation of a vibration other than that produced during the initial capture. Such vibrations can either be excited through IVR following resonant cap- ture or by thermal excitation. This process is expected to lead to a reduction in the annihilation rate since the positron will spend less time on the molecule.

An important consideration in such an inelastic process is how the resonant $Z_{\text {eff }}$ is affected by the binding energy. If the positron is in a weakly bound state, many vibrational modes will have sufficient energy to eject it; while for deeply bound states, fewer modes are able to do this. Thus, if inelastic escape channels were present, one would expect an additional dependence of the $Z_{\text {eff }}$ on $\varepsilon_{b}$, beyond the scaling with $g$. However, this contradicts a number of experimental results. For example, the $\mathrm{C}-\mathrm{H}$ stretch-peak magnitudes for the first and second bound states of tetradecane and hexadecane strictly follow the $g$ scaling with no additional dependence on $\varepsilon_{b}$. Furthermore, the $Z_{\text {eff }}$ at the $\mathrm{C}-\mathrm{H}$ stretch peaks for nearly all molecules studied adhere strictly the $Z_{\text {eff }} / g$ $\propto N^{4.1}$ scaling, with relatively little deviation due to their disparate binding energies. This appears to rule out inelastic escape channels as being generally important in determining $Z_{\text {eff }}$ values.

\section{Fluorine-substituted alkanes}

There is, however, one notable exception. As shown in Fig. 27, partially fluorinated alkanes, such as the 1-fluoroalkanes, deviate significantly from the scaling of Eq. (59). The data indicate that the substitution of a single fluorine atom for a terminal hydrogen in a given alkane reduces the height of the $\mathrm{C}-\mathrm{H}$ stretch peak in $Z_{\text {eff }}$ by as much as an order of magnitude. The C-F stretch mode might be expected to play a significant role in this apparently inelastic process since the cross section for positron-impact excitation of the C-F stretch vibration in $\mathrm{CF}_{4}$ is unusually large (Sullivan, Gilbert, Marler, Barnes, et al., 2002; Marler et al., 2006).

Such an inelastic channel can significantly reduce the magnitude of $Z_{\text {eff }}$ by increasing the total post-capture escape rate. In this case [cf. Eq. (42)],

$$
Z_{\mathrm{eff}}(\nu) \propto \frac{\Gamma_{\nu}^{e}}{\Gamma_{\nu}^{a}+\Gamma_{\nu}^{e}+\Gamma_{\nu}^{i}},
$$

where $\Gamma_{\nu}^{i}$ is the inelastic escape rate (e.g., via deexcitation of mode $n$ with energy $\omega_{n}$ ), so that increasing $\Gamma_{\nu}^{i}$ reduces $Z_{\text {eff }}$. This process requires that the multimode state $\nu$ contains quasidegenerate components in which mode $n$ is excited. This restriction imposes a threshold at $\varepsilon=\omega_{n}-\varepsilon_{b}$, above which $Z_{\text {eff }}$ is reduced.

As shown in Fig. 28, the $Z_{\text {eff }}$ spectra for 1- and 2,2fluoropropane display just such a suppression at larger incident positron energies as compared with the analogous hydrogenated compounds. In 1-fluoropropane, the $\mathrm{C}-\mathrm{F}$ stretch annihilation resonance is expected to occur at $\sim 90 \mathrm{meV}$, assuming $\varepsilon_{b}=30 \mathrm{meV}$ and $\omega_{\mathrm{CF}} \approx 120 \mathrm{meV}$ (Guirgis et al., 1999). In 2,2-difluoropropane, the C-F stretch peak is expected to occur at $\sim 125 \mathrm{meV}$, assuming $\varepsilon_{b}=25 \mathrm{meV}$ and $\omega_{\mathrm{CF}} \approx 150 \mathrm{meV}$ (Durig et al., 1981). In both cases, the suppression of $Z_{\text {eff }}$ occurs near these 


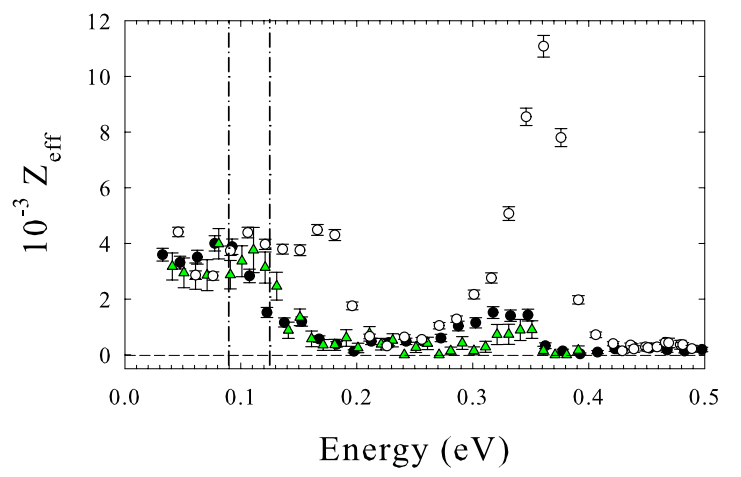

FIG. 28. (Color online) The $Z_{\text {eff }}$ spectra for $(\bigcirc)$ propane and (-) 1-fluoropropane (Young and Surko, 2008b) and $(\triangle)$ 2,2difluoropropane (Gilbert et al., 2002). The vertical dot-dashed lines indicate the expected energies of C-F stretch resonances and escape thresholds in each molecule, based on C-F stretch mode energies from Durig et al. (1981) and Guirgis et al. (1999). See text for details.

threshold energies (shown by dot-dashed lines in Fig. 28).

As discussed by Young and Surko (2008b), there are similar decreases in the magnitudes of the $Z_{\text {eff }}$ at higher incident positron energies in the larger partially fluorinated alkanes, 1-fluorobutane, 1-fluorohexane, and 1-fluorononane, relative to the analogous hydrogenated molecules. However, there is also an increase in the magnitudes of the $Z_{\text {eff }}$ spectra at smaller positron energies, making the thresholds for escape-channel behavior less distinct. The behavior of 1-fluorononane is more difficult to explain. The positron binding energy of $160 \mathrm{meV}$ in this molecule would appear to preclude the C-F stretch mode $\left[\omega_{n} \lesssim 127 \mathrm{meV}\right.$ (Crowder and Lightfoot, 1983)] from acting as an inelastic escape channel. However, a decrease in annihilation is observed at higher energies nonetheless (Young and Surko, 2008b). This is currently not understood.

\section{Effects of molecular temperature on $Z_{\text {eff }}$}

For molecules at finite temperatures, the energy for positron detachment which suppresses $Z_{\text {eff }}$ can be supplied by preexisting thermally excited modes rather than those excited in the attachment process. Thus $Z_{\text {eff }}$ might be expected to increase significantly with decreasing molecular temperature. At one point, it was thought this could explain the empirical observation that $Z_{\mathrm{eff}} \propto(2 n$ $+2) \exp \left(\varepsilon_{b} / k_{B} T\right)$ in alkane molecules (Barnes, 2004). A qualitatively different effect of varying molecular temperature was suggested by Nishimura and Gianturco (2005b). They proposed that vibrational excitation and the associated change in molecular geometry could be required to induce or increase positron binding to molecules, and this, in turn, could affect the activation of annihilation resonances. In this model, one would expect that increasing the molecular temperature should result in an increase in $Z_{\text {eff }}$.

Experiments were done to test these ideas using a specially constructed cold cell so that $Z_{\text {eff }}$ spectra could be

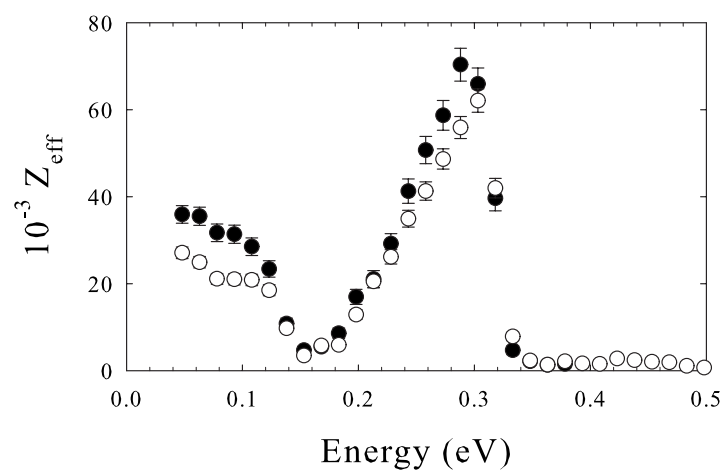

FIG. 29. Energy-resolved measurements of $Z_{\text {eff }}$ for pentane $\left(\mathrm{C}_{5} \mathrm{H}_{12}\right)$ at $300 \mathrm{~K}(\bigcirc)$ and $153 \mathrm{~K}(\bullet)$ using a cold cell (Young and Surko, 2008a).

measured at different molecular temperatures (Young and Surko, 2008a). The apparatus is described in Sec. III.E. Care was taken to ensure that the test gas in this flowing-gas system actually cooled to the ambient temperature of the cold cell. In addition, the test-gas pressure was maintained a safe margin below the equilibrium vapor pressure at each temperature in order to avoid condensation on surfaces near and inside the cell. The spectra for pentane at 153 and $300 \mathrm{~K}$ are shown in Fig. 29 . There is only a small $(\sim 10 \%)$ increase in the magnitude of the $\mathrm{C}-\mathrm{H}$ stretch resonance with the change in molecular temperature, while at lower incident positron energies, there is a somewhat larger increase in $Z_{\text {eff }}$ (i.e., $\sim 30 \%$ ). Similar results were obtained for heptane at 195 and $300 \mathrm{~K}$, but the increase in the low-energy portion of the spectrum was somewhat larger $(\sim 50 \%)$ (Young and Surko, 2008a). The spectra of both molecules indicate that their binding energies do not change with changes in the molecular temperature.

These experimental results indicate that there is clearly no Boltzmann-factor-like dependence of $Z_{\text {eff }}$ as that considered by Barnes (2004). If there were, the pentane $\mathrm{C}-\mathrm{H}$ stretch peak would have grown by a factor of 10. These results also tend to rule out the suggestion made by Nishimura and Gianturco (2005b) for $\mathrm{C}_{2} \mathrm{H}_{2}$, $\mathrm{C}_{2} \mathrm{H}_{4}$, and $\mathrm{C}_{2} \mathrm{H}_{6}$ that thermal deformation of the molecular bonds can enhance significantly the binding energy and hence increases the rate of VFR-mediated positron attachment and annihilation. The data show the opposite trend, namely, that increasing the molecular temperature leads to smaller values of $Z_{\text {eff }}$.

These findings also confirm other results indicating the absence of thermally excited escape channels. For thermally excited modes to provide effective escape, their energies must exceed the positron binding energy. As the binding energy increases (e.g., with alkane size), one would expect these channels to be switched off. Hence thermally activated detachment would produce an additional dependence of the resonant $Z_{\text {eff }}$ on $\varepsilon_{b}$ beyond the $g$ factor. Such an effect is not apparent in the alkanes, where the empirical scaling is described by Eq. (59) for both the positron ground and first excited states. 
A question remains as to why the temperature effect appears to be stronger at low impact energies than at high energies, yet the $g$-normalized spectra for alkanes at $300 \mathrm{~K}$ (cf. Fig. 24) remain self-similar.

\section{F. Other IVR-related phenomena}

While the $N^{4.1}$ scaling provides strong evidence of IVR, there are still significant questions as to how the IVR, induced by positron capture, proceeds in large molecules. We discuss here a few of the outstanding issues.

The partially fluorinated alkanes (Sec. V.E.1) provide a clear example of postcapture vibrational energy transfer, which suppresses the $Z_{\text {eff }}$ spectra due to inelastic detachment. The so-called "intermediate" multimode state containing the excited C-F stretch mode is suggestive of a tiered IVR model, in which vibrational energy redistribution occurs incrementally through an evergrowing set of dark states (Nesbitt and Field, 1996). This could explain why only a few multimode excitations involving the C-F stretch (out of all possible excitations) have such a disproportionate influence. A similar tiered model has been used to describe laser-excited vibrational dynamics in phenols (Yamada et al., 2007) and to calculate IVR rates for acetylenic stretch modes (Stuchebrukhov and Marcus, 1993).

In general, the haloalkanes have larger values of thermal $Z_{\text {eff }}$ at $300 \mathrm{~K}$ than their hydrogenated counterparts. There is also evidence that thermal values of $Z_{\text {eff }}$ for partially deuterated benzenes and other substituted benzenes are similarly enhanced (Iwata et al., 1995). These observed increases in $Z_{\text {eff }}$ may be due to the increase in the density of vibrational "dark" states. Similar physics may account for the large thermal $Z_{\text {eff }}$ values in $\mathrm{CCl}_{4}$ and $\mathrm{CBr}_{4}$ (9000 and 40000 , respectively).

It is not clear at present what makes a "good" vibrational doorway state. In most large hydrocarbons, it is only the fundamental vibrations that appear to produce VFR; however, there are exceptions. Shown in Fig. 30 is the $Z_{\text {eff }}$ spectrum of benzene, shifted upward by its binding energy and compared with the infrared absorption spectrum. Note the distinct peak at $\sim 235 \mathrm{meV}$ in the shifted $Z_{\text {eff }}$ spectrum. While there are no nearby fundamental vibrations, there are two IR-active combination vibrations at 227 and $244 \mathrm{meV}$ in the IR spectrum. Thus, the additional peak in $Z_{\text {eff }}$ appears to be evidence of unusually strong capture into multimode doorways, likely enhanced by IVR.

In elucidating the role of IVR it is of interest to compare propane and cyclopropane. Figure 31 shows that transforming the propane molecule into a ring reduces the C-H stretch peak by a factor of 3, approximately in agreement with the $N^{4}$ scaling in Eq. (59). At the same time the plateau at lower energies is narrowed so that only one broad peak remains. However, its magnitude in cyclopropane is practically the same as in propane. Thus the cyclopropane spectrum does not obey the selfsimilar scaling observed in the alkanes. It is possible that

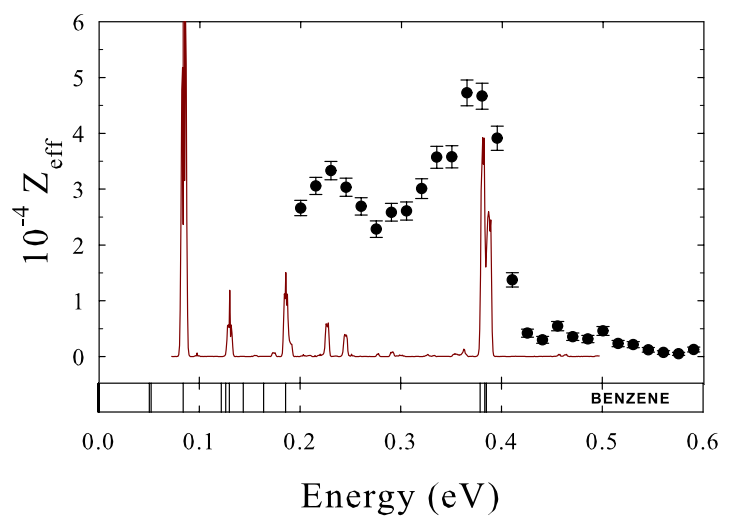

FIG. 30. (Color online) Energy-resolved $Z_{\text {eff }}(\bullet)$ and infrared absorption (solid curve) for benzene. The $Z_{\text {eff }}$ spectrum has been shifted upward by the binding energy $\left(\varepsilon_{b}=150 \mathrm{meV}\right)$ for direct comparison. The normalization of the IR absorption (Linstrom and Mallard, 2005) is arbitrary. Vertical lines indicate the positions of the vibrational modes.

the low-energy peak contains mode-based VFRs, with little enhancement due to IVR. The reduction in the magnitude of the $\mathrm{C}-\mathrm{H}$ peak may be due to the absence of low-frequency modes in cyclopropane. This is consistent with Table III which shows that cyclopropane has a markedly lower vibrational density at the $\mathrm{C}-\mathrm{H}$ stretch energy as compared with propane.

As shown in Fig. 31, there is an identifiable feature in cyclopropane at $\sim 250 \mathrm{meV}$ (i.e., in the gap between the

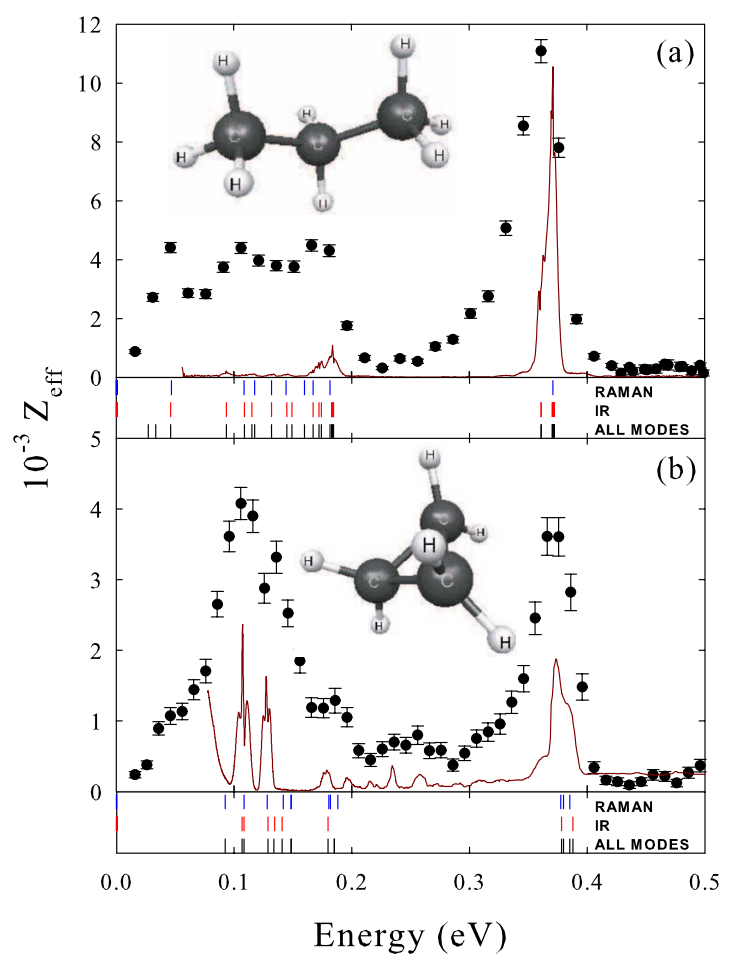

FIG. 31. (Color online) Energy-resolved $Z_{\text {eff }}(\bullet)$ for (a) propane and (b) cyclopropane (Barnes et al., 2006). The solid curves are the normalized IR absorption spectra, and the vertical bars below each plot show the vibrational modes (Linstrom and Mallard, 2005). Shown as insets are the molecular structures. 
C-H stretch and lower-energy modes) that does not appear in propane or larger hydrocarbons. It occurs in the energy range where there are peaks in the IR spectrum and it is likely due to combination and overtone vibrations (similar to that observed in benzene). This again points to the possibility that appreciable IR coupling is a predictor of the strength of multimode doorways in large molecules. In cyclopropane this peak is not particularly enhanced (e.g., relative to the C-H stretch resonance), and looks closer to the effects due to combination and overtone VFRs observed in small molecules (see Sec. IV, e.g., ethylene, Fig. 17). This phenomenon also bears further scrutiny.

\section{G. Large molecule summary}

There are several defining characteristics of the $Z_{\text {eff }}$ spectra of large molecules. They exhibit a series of peaks, the positions of which bear close resemblance to those of the fundamental vibrations, downshifted by the positron-molecule binding energy. The amplitudes of these resonances grow rapidly with molecular size but exhibit a relatively weak dependence on binding energy and incident positron energy via the factor $g$. The amplitudes of the $\mathrm{C}-\mathrm{H}$ stretch resonances in hydrocarbons obey the empirical scaling of Eq. (59). This scaling likely reflects the rapid increase in the number of vibrational degrees of freedom of the molecule with molecular size. It suggests that the VFR are enhanced by the IVR process. However, the extent of this IVR appears to be limited and does not reach the statistically complete final state. Inelastic escape channels appear to be relatively unimportant, at least in the hydrocarbons studied thus far.

Our theoretical understanding of annihilation processes in large molecules is not nearly as well developed as it is for small molecules. Qualitatively, a positron appears to excite a vibrational fundamental (or in some cases, such as benzene, a combination or overtone) and populates a doorway resonance. The vibrational energy imparted to the molecule can then flow to some set of quasidegenerate dark states. However, if this picture is correct, there must be an operative escape channel. If not, all VFR will give approximately the same contribution to the annihilation rate. Considering the available experimental evidence, it seems plausible that the resonant $Z_{\text {eff }}$ magnitudes are determined by a balance between diffusion to some (limited) set of dark states, and positron ejection through either the doorway state that it entered or other nearby doorway states in what might be termed a "quasielastic" escape process.

Beyond this qualitative picture, there are an assortment of relatively poorly understood effects. The most prominent of them is arguably the self-similar spectra observed in alkanes. There is also the occasional occurrence of multimode VFR, and the inelastic detachment observed in partially fluorinated alkanes. These effects provide tantalizing hints of the many types of complex internal dynamics that can be responsible for VFRenhanced annihilation depending upon the particular

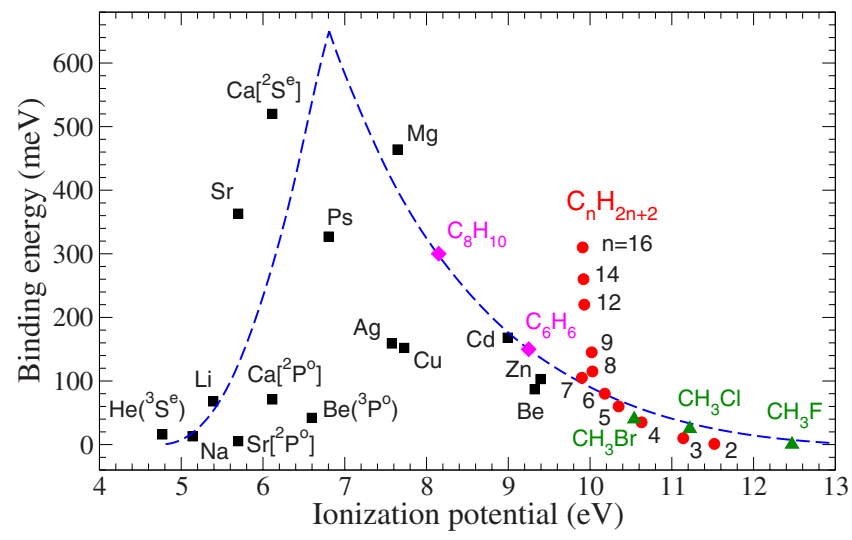

FIG. 32. (Color online) Binding energies of positron-atom and positron-molecule complexes as a function of their ionization potential. Calculations: squares, various atoms (Mitroy et al., 2002; Bromley and Mitroy, 2010); dashed curve, model alkali atom (Mitroy et al., 1999). Experiment: circles, alkanes with $n$ carbons; diamonds, the aromatic molecules, benzene and naphthalene; triangles, methyl halides (see Secs. IV-VI). Adapted from Danielson et al., 2009.

situation. At a minimum, further investigations are warranted. For example, experiments with higher positron energy resolution would be useful to determine additional details of the processes involved.

\section{POSITRON-MOLECULE BINDING ENERGIES}

\section{A. Relation to molecular properties}

As described in Secs. IV and V, positron-molecule binding energies have now been measured or estimated for about 30 molecules (Gilbert et al., 2002; Barnes et al., 2003, 2006; Young and Surko, 2008b, 2008c). These data were analyzed recently by Danielson et al. (2009) with a view toward determining which molecular parameters affect $\varepsilon_{b}$. Table VI lists the available binding energies together with molecular dipole polarizabilies $\alpha_{d}$, dipole moments $\mu$, and ionization energies $E_{i}$.

Figure 32 shows the experimental binding energies for alkanes, aromatic molecules, and methyl halides and calculated binding energies for several atoms as a function of their ionization potential. The magnitudes of $\varepsilon_{b}$ calculated for atoms and measured for molecules are quite similar (i.e., $\varepsilon_{b} \leqslant 0.5 \mathrm{eV}$ ). The binding energies for smaller alkanes, aromatics, and methyl halides follow a "model atom" curve (Mitroy et al., 1999). However, the ionization energies for alkanes with more than $n=7$ carbons remain practically constant, while the positron binding energies continue to grow with $n$.

The dipole polarizability $\alpha_{d}$ characterizes the strength of the long-range positron-molecule attraction. Figure 33 shows positron binding energies for molecules as a function of $\alpha_{d}$. Also shown are the theoretical values for three atoms, Be, Zn, and Cd (Mitroy et al., 2002). This figure suggests an approximately linear relationship between $\varepsilon_{b}$ and $\alpha_{d}$ for groups of homologous species. Based on this, the analysis of Danielson et al. (2009) 
TABLE VI. Positron binding energies, normalized thermal annihilation rates $Z_{\mathrm{eff}}^{\mathrm{th}} / Z$, and physical parameters for selected molecules.

\begin{tabular}{|c|c|c|c|c|c|c|c|c|}
\hline Name & Formula & $\begin{array}{c}\varepsilon_{b}{ }^{\mathrm{a}} \\
(\mathrm{meV})\end{array}$ & $\begin{array}{c}\varepsilon_{b}^{\mathrm{b}} \\
(\mathrm{meV})\end{array}$ & $Z$ & $Z_{\mathrm{eff}}^{\mathrm{th}} / Z^{\mathrm{c}}$ & $\begin{array}{c}\alpha_{d}^{\mathrm{d}} \\
\left(\AA^{3}\right)\end{array}$ & $\begin{array}{l}\mu^{\mathrm{d}} \\
\text { (D) }\end{array}$ & $\begin{array}{c}E_{i}^{\mathrm{d}} \\
(\mathrm{eV})\end{array}$ \\
\hline \multicolumn{9}{|c|}{ Alkanes and related molecules } \\
\hline Ethane & $\mathrm{C}_{2} \mathrm{H}_{6}$ & $>0$ & -14 & 18 & 37 & 4.4 & 0 & 11.5 \\
\hline Propane & $\mathrm{C}_{3} \mathrm{H}_{8}$ & 10 & 10 & 26 & 135 & 6.3 & 0.1 & 11.1 \\
\hline Butane & $\mathrm{C}_{4} \mathrm{H}_{10}$ & 35 & 31 & 34 & 330 & 8.1 & 0 & 10.6 \\
\hline Pentane & $\mathrm{C}_{5} \mathrm{H}_{12}$ & 60 & 54 & 42 & 900 & 10.0 & 0 & 10.4 \\
\hline Hexane & $\mathrm{C}_{6} \mathrm{H}_{14}$ & 80 & 77 & 50 & 2400 & 11.8 & 0 & 10.2 \\
\hline Heptane & $\mathrm{C}_{7} \mathrm{H}_{16}$ & 105 & 100 & 58 & 4200 & 13.7 & 0 & 9.9 \\
\hline Octane & $\mathrm{C}_{8} \mathrm{H}_{18}$ & 115 & 123 & 66 & 8800 & 15.5 & 0 & 10.0 \\
\hline Nonane & $\mathrm{C}_{9} \mathrm{H}_{20}$ & 145 & 146 & 74 & 8700 & 17.4 & 0 & 10.0 \\
\hline Dodecane & $\mathrm{C}_{12} \mathrm{H}_{26}$ & 220 & 214 & 98 & 18000 & 22.9 & 0 & 9.9 \\
\hline Tetradecane & $\mathrm{C}_{14} \mathrm{H}_{30}$ & 260 & 261 & 114 & & 26.6 & 0 & 9.9 \\
\hline Hexadecane & $\mathrm{C}_{16} \mathrm{H}_{34}$ & 310 & 306 & 130 & & 30.3 & 0 & 9.9 \\
\hline Butane-d10 & $\mathrm{C}_{4} \mathrm{D}_{10}$ & 35 & 31 & 34 & & 8.1 & 0 & \\
\hline Nonane-d20 & $\mathrm{C}_{9} \mathrm{D}_{20}$ & 145 & 146 & 74 & 8700 & 17.4 & 0 & \\
\hline Acetylene & $\mathrm{C}_{2} \mathrm{H}_{2}$ & $>0$ & -28 & 14 & 230 & 3.3 & 0 & 11.4 \\
\hline Ethylene & $\mathrm{C}_{2} \mathrm{H}_{4}$ & 20 & -17 & 16 & 75 & 4.2 & 0 & 10.5 \\
\hline Isopentane & $\mathrm{C}_{5} \mathrm{H}_{12}$ & 60 & 57 & 42 & 1200 & 10.0 & 0.1 & 10.3 \\
\hline Cyclopropane & $\mathrm{C}_{3} \mathrm{H}_{6}$ & 10 & 0.7 & 24 & & 5.7 & 0 & 9.9 \\
\hline Cyclohexane & $\mathrm{C}_{6} \mathrm{H}_{12}$ & 80 & 68 & 48 & 420 & 11.1 & 0 & 9.9 \\
\hline \multicolumn{9}{|c|}{ Aromatics } \\
\hline Benzene & $\mathrm{C}_{6} \mathrm{H}_{6}$ & 150 & 149 & 42 & 360 & 10.4 & 0 & 9.3 \\
\hline Benzene-d6 & $\mathrm{C}_{6} \mathrm{D}_{6}$ & 150 & 149 & 42 & 730 & 10.4 & 0 & 9.3 \\
\hline Naphthalene & $\mathrm{C}_{10} \mathrm{H}_{8}$ & 300 & 296 & 68 & 7300 & 16.6 & 0 & 8.2 \\
\hline \multicolumn{9}{|c|}{ Alcohols } \\
\hline Methanol & $\mathrm{CH}_{3} \mathrm{OH}$ & 2 & 5 & 18 & 84 & 3.3 & 1.7 & 10.9 \\
\hline Ethanol & $\mathrm{C}_{2} \mathrm{H}_{5} \mathrm{OH}$ & 45 & 27 & 26 & & 5.1 & 1.7 & 10.5 \\
\hline \multicolumn{9}{|c|}{ Partially halogenated hydrocarbons } \\
\hline Methyl fluoride & $\mathrm{CH}_{3} \mathrm{~F}$ & $>0$ & -3 & 18 & 77 & 2.4 & 1.85 & 12.9 \\
\hline Methyl chloride & $\mathrm{CH}_{3} \mathrm{Cl}$ & 25 & 23 & 26 & 580 & 4.4 & 1.9 & 11.2 \\
\hline Methyl bromide & $\mathrm{CH}_{3} \mathrm{Br}$ & 40 & 35 & 44 & & 5.6 & 1.8 & 10.5 \\
\hline 1-fluoropropane & $\mathrm{C}_{3} \mathrm{H}_{7} \mathrm{~F}$ & 30 & 45 & 34 & & 6.0 & 2.0 & 11.3 \\
\hline 2,2-difluoropropane & $\mathrm{C}_{3} \mathrm{H}_{6} \mathrm{~F}_{2}$ & 25 & 51 & 42 & 190 & 5.9 & 2.4 & 11.4 \\
\hline 1-fluorobutane & $\mathrm{C}_{4} \mathrm{H}_{9} \mathrm{~F}$ & 70 & $27^{\mathrm{e}}$ & 42 & & 7.8 & & \\
\hline 1-fluorohexane & $\mathrm{C}_{6} \mathrm{H}_{13} \mathrm{~F}$ & 105 & $73^{\mathrm{e}}$ & 58 & 46000 & 11.5 & & \\
\hline 1-fluorononane & $\mathrm{C}_{9} \mathrm{H}_{19} \mathrm{~F}$ & 160 & $141^{\mathrm{e}}$ & 82 & & 17.0 & & \\
\hline 1-chlorohexane & $\mathrm{C}_{6} \mathrm{H}_{13} \mathrm{Cl}$ & 175 & 138 & 66 & & 13.6 & 2.0 & 10.3 \\
\hline
\end{tabular}

${ }^{a}$ Measured in energy-resolved annihilation experiments (Young and Surko, 2008b, 2008c).

${ }^{\mathrm{b}}$ Predicted by Eq. (61).

${ }^{\mathrm{c}} Z_{\mathrm{eff}}^{\mathrm{th}}$ is measured at room temperature with thermalized positrons (Iwata et al., 1995; Iwata, 1997).

${ }^{\mathrm{d}}$ Polarizabilities, dipole moments, and ionization energies from McClellan (1963), Miller (1990), and Lide (2000).

${ }^{\mathrm{e}}$ Lacking values of $\mu$ for these species, the predictions of Eq. (61) are lower bounds on $\varepsilon_{b}$.

began with a linear fit of $\varepsilon_{b}$ to $\alpha_{d}$ for alkanes. However, as seen in Fig. 33, this fit generally underestimates the binding energies for other classes of molecules, most no- tably those with permanent dipole moments and aromatic molecules. This motivated the inclusion of two additional parameters in the analysis, namely, the mo- 


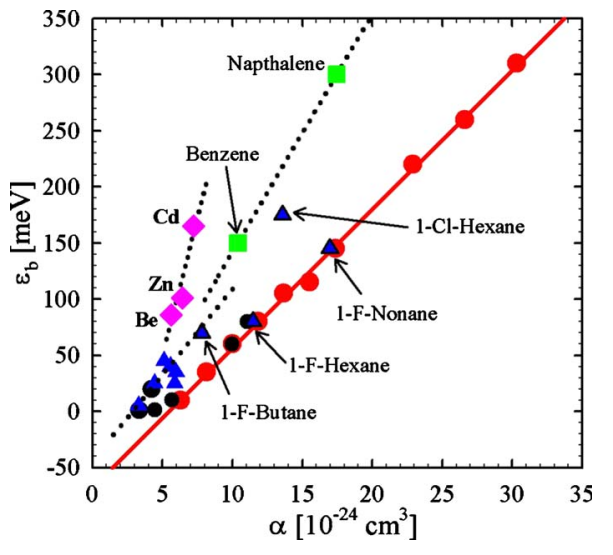

FIG. 33. (Color online) Measured positron binding energies $\varepsilon_{b}$ as a function of the dipole polarizability $\alpha_{d}$ : large circles, alkane molecules used in the linear fit shown by the solid line; smaller circles, alkane-related molecules; triangles, molecules with permanent dipole moments; squares, aromatics with $\pi$ bonds. Dotted lines, guides to show the linearity of $\varepsilon_{b}$ for the different series; diamonds, calculated $\varepsilon_{b}$ for the atoms $\mathrm{Be}, \mathrm{Zn}$, and Cd (Mitroy et al., 2002), shown for comparison. From Danielson et al., 2009.

lecular dipole moment $\mu$ and the number of $\pi$ bonds $N_{\pi}$ for aromatic molecules. Such use of the bonds is similar to the approach used by Miller (1990) to parametrize the molecular polarizability.

The resulting fitting function, using the numerical values of the molecular parameters as listed in Table VI, is (Danielson et al., 2009)

$$
\varepsilon_{b}=12.4\left(\alpha+1.6 \mu+2.4 N_{\pi}-5.6\right)[\mathrm{meV}],
$$

where $\varepsilon_{b}$ is in meV, $\alpha_{d}$ is in units of $\AA^{3}$, and $\mu$ is in units of Debye. This expression can be viewed as a lowestorder Taylor expansion of $\varepsilon_{b}$ as a function of the variables $\alpha_{d}, \mu$, and $N_{\pi}$. This fit to the binding energy data is shown in Fig. 34. Generally, the agreement between the predictions of Eq. (61) and the measurements is quite good. The most significant discrepancies are 1-

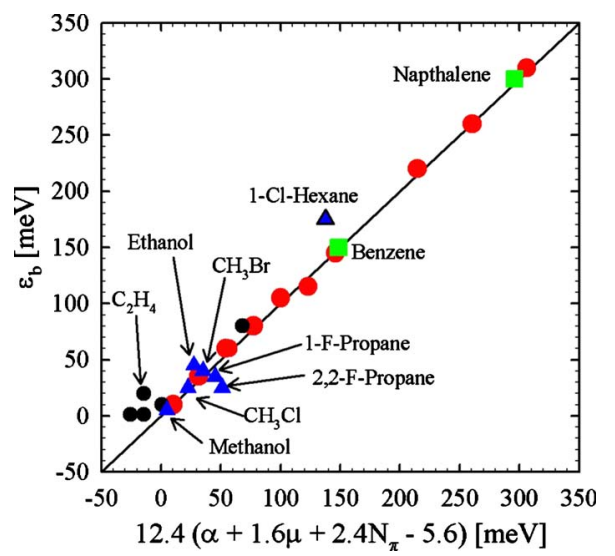

FIG. 34. (Color online) Binding energy from the fit Eq. (61) (solid line) using the polarizability $\alpha_{d}$, dipole moment $\mu$, and the number of $\pi$ bonds $N_{\pi}$ for aromatic molecules. Symbols as in Fig. 33. From Danielson et al., 2009. chlorohexane, acetylene $\left(\mathrm{C}_{2} \mathrm{H}_{2}\right)$, ethylene $\left(\mathrm{C}_{2} \mathrm{H}_{4}\right)$, and 2,2-difluoropropane. For all but 2,2-difluoropropane, Eq. (61) underestimates the binding energy. These molecules probably possess some additional attraction beyond that described by Eq. (61). With the exception of 1-chlorohexane and 2,2-difluoropropane, the outliers all have double and triple bonds. The addition of the $N_{\pi}$ term in Eq. (61), which improves agreement for the aromatic species, overestimates $\varepsilon_{b}$ for these smaller molecules. Thus, while a similar effect may well be operating in small molecules with $\pi$ bonds, its magnitude appears to be smaller than in the aromatics.

According to Eq. (61), binding is assured if either $\alpha_{d}$ $>5.4 \AA^{3}$ or $\mu>3.6 \mathrm{D}$. The first of these conditions is similar to that for positron binding to a model "alkali atom," namely, $\alpha_{d}>3.5 \AA^{3}$ (Mitroy et al., 1999). The second condition can be compared with the theoretical critical value $\mu_{c}=1.625 \mathrm{D}$, beyond which the dipole supports an infinite series of bound states (Crawford, 1967). Probably more relevant is a "practical" value $\mu_{c}$ $\approx 2.5 \mathrm{D}$ required to obtain binding energies $\geqslant 1 \mathrm{meV}$ in negative ions (i.e., electron-molecule bound states) (Abdoul-Carime and Desfrançois, 1998); see below. These comparisons show that the threshold values of $\alpha_{d}$ and $\mu$ from Eq. (61) appear to be quite reasonable.

Another feature of Eq. (61), which is supported by observations (Table VI), is that it predicts that the binding energies for fully deuterated hydrocarbons are close to their hydrogenated analogs (cf. deuterated butane, benzene, and nonane) and that isomers have similar values of $\varepsilon_{b}$ (e.g., pentane vis-á-vis isopentane).

For the species studied to date, the largest binding energies for a given number of atoms are seen in the aromatic molecules. A study of larger species (i.e., polycyclic aromatic hydrocarbons), such as the three- and four-ring variants anthracene and pyrene, will be of interest. If $\varepsilon_{b}$ increases beyond the energy of the $\mathrm{C}-\mathrm{H}$ stretch mode (i.e., the highest-energy mode), the resonant energy threshold in these molecules becomes negative and the VFR of the vibrational fundamentals become inaccessible to the positron. This has already been observed in deuterated naphthalene (Young and Surko, 2008b). Nevertheless, the annihilation spectra for these molecules may still exhibit VFRs associated with positronically excited states or those where the positron excites higher-lying overtone and combination vibrations.

For molecules with similar chemical composition, $\alpha_{d}$ increases with molecular size. However, the linear increase in $\varepsilon_{b}$ with $\alpha_{d}$ predicted by Eq. (61) is expected to saturate at some point (e.g., when the positron de Broglie wavelength becomes smaller than the size of the molecule). The data in Figs. 33 and 34 indicate that the experiments have not yet reached this limit.

The predictions of Eq. (61) can also be compared with available calculations for positron binding to molecules. All of the molecules listed in Table VII are predicted to bind positrons in agreement with the theoretical calculations. The absolute values of $\varepsilon_{b}$ predicted by Eq. (61) 
TABLE VII. Comparisons of theoretical predictions for positron-molecule binding energies with those of Eq. (61).

\begin{tabular}{lccccc}
\hline \hline Name & Formula & $\begin{array}{c}\alpha_{d} \\
\left(\AA^{3}\right)\end{array}$ & $\begin{array}{c}\mu \\
(\mathrm{D})\end{array}$ & $\begin{array}{c}\varepsilon_{b}{ }^{\mathrm{a}} \\
(\mathrm{meV})\end{array}$ & $\begin{array}{c}\varepsilon_{b}{ }^{\mathrm{b}} \\
(\mathrm{meV})\end{array}$ \\
\hline Hydrogen cyanide & $\mathrm{HCN}$ & 2.5 & 3.0 & 21 & 35 \\
Formaldehyde & $\mathrm{H}_{2} \mathrm{CO}$ & 2.8 & 2.3 & 12 & 19 \\
Urea & $\left(\mathrm{NH}_{2}\right)_{2} \mathrm{CO}$ & 9.7 & 4.6 & 140 & 13 \\
Acetone & $\left(\mathrm{CH}_{3}\right)_{2} \mathrm{CO}$ & 6.4 & 2.9 & 66 & 4 \\
Lithium hydride & $\mathrm{LiH}$ & 3.8 & 5.9 & 94 & 1000 \\
\hline \hline
\end{tabular}

${ }^{\mathrm{a}}$ Predicted from Eq. (61).

${ }^{b}$ Values from quantum-chemistry calculations: $\mathrm{HCN}$ (Chojnacki and Strasburger, 2006), $\mathrm{H}_{2} \mathrm{CO}$ (Strasburger, 2004), urea and acetone (Tachikawa et al., 2003), and LiH (Mella et al., 2000; Strasburger, 2001; Bubin and Adamowicz, 2004).

for $\mathrm{HCN}$ and formaldehyde $\left(\mathrm{H}_{2} \mathrm{CO}\right)$ agree to within a factor of 2 with the calculations. For urea and acetone Eq. (61) predicts much larger values than the calculated $\varepsilon_{b}$. This confirms the expectation (Strasburger, 2004) that the binding energies calculated by Tachikawa et al. (2003) for urea and acetone are underestimated.

For $\mathrm{LiH}$, the calculated $\varepsilon_{b}$ is ten times greater than the prediction of Eq. (61). This is also true for other alkali hydrides, $\mathrm{NaH}, \mathrm{KH}$, and $\mathrm{RbH}$, where the calculations give $\varepsilon_{b} \gtrsim 1 \mathrm{eV}$ (Buenker et al., 2005; Gianturco et al., 2006). This discrepancy is due to the fact that Eq. (61) is based on binding energies for molecules with relatively large ionization energies. The physical picture of such bound states gained from positron calculations for atoms (Mitroy et al., 2002) describes them as the positron moving in the attractive potential of the neutral molecule. In contrast, the ionization energy of $\mathrm{LiH}$ and the other alkali hydrides is close to the binding energy of the Ps atom (e.g., $E_{i}-E_{\mathrm{Ps}}=1.1 \mathrm{eV}$ for $\mathrm{LiH}$ ). In this case, the calculations indicate that the relevant physical picture is closer to that of a PsH complex attached to the positively charged metal ion (Buenker et al., 2005, 2006); cf. Fig. 8. Thus it is not surprising that there is a discrepancy between the predictions of the Eq. (61) and calculations of $\varepsilon_{b}$ for $\mathrm{LiH}$.

Equation (61) can be used to identify candidate molecules for further theoretical and experimental studies. Theoretical calculations favor molecules with small numbers of atoms and simple electronic structures. Experimental studies require species with vapor number densities $\geqslant 10^{-7}$ amagat at moderate temperatures (e.g., $T \leqslant 500{ }^{\circ} \mathrm{C}$ ) that are stable at this temperature and not deleterious to the vacuum system. Most convenient for study are small molecules with relatively large binding energies (e.g., $\varepsilon_{b} \geqslant 20 \mathrm{meV}$ ).

Recently Danielson et al. (2010) measured $\varepsilon_{b}$ for several molecules of this type. For carbon disulfide $\mathrm{CS}_{2}$ $\left(\alpha_{d}=8.8 \AA^{3}\right.$ and $\left.\mu=0\right)$, the measured value $\varepsilon_{b}=75 \mathrm{meV}$ is a factor of 2 greater than $40 \mathrm{meV}$, predicted by Eq. (61) neglecting $\pi$ bonds. Other molecules studied, which were chosen for their simplicity and relatively large dipole moments, include acetaldehyde $\mathrm{CH}_{3} \mathrm{CHO}\left(\alpha_{d}\right.$
$=4.6 \AA^{3}, \quad \mu=2.75 \mathrm{D}, \quad$ and $\left.\quad \varepsilon_{b}=90 \mathrm{meV}\right), \quad$ acetone $\left(\mathrm{CH}_{3}\right)_{2} \mathrm{CO}\left(\alpha_{d}=6.4 \AA^{3}, \mu=2.9 \mathrm{D}\right.$, and $\left.\varepsilon_{b}=173 \mathrm{meV}\right)$, and acetonitrile $\mathrm{CH}_{3} \mathrm{CN}\left(\alpha_{d}=4.4 \AA^{3}, \mu=3.9 \mathrm{D}\right.$, and $\varepsilon_{b}$ $=180 \mathrm{meV}$ ). All three have binding energies significantly larger than those predicted by Eq. (61). In this previously unexplored regime in which $\mu>2 \mathrm{D}$, the dependence on both $\mu$ and $\alpha_{d}$ is much stronger than that given by Eq. (61). These data provide a significant new opportunity to make quantitative comparisons between theory and experiment. It is hoped that they will stimulate new theoretical calculations. In this regard, a recent CI calculation for acetonitrile by Tachikawa et al. (2010) predicted $\varepsilon_{b}=135 \mathrm{meV}$, which is within $30 \%$ of the experimental value.

Equation (61) could also be useful in describing the behavior of low-energy positrons in a wide range of chemical environments, including biological systems (e.g., in PET analysis) and in materials science in conjunction with studies of insulators using techniques such as ACAR and positron annihilation lifetime spectroscopy.

\section{B. Comparison with negative ions of molecules and clusters}

Unlike positron-molecule complexes, negative molecular ions have been studied extensively. They can be separated into two classes. There is a class of stable molecular anions with large electron affinities $\sim 1-3 \mathrm{eV}$ (Rienstra-Kiracofe et al., 2002). In these anions, the excess electron occupies an unfilled orbital in the valence shell of the molecule, and they have no positron analog.

A second arguably less well-studied class of negative ions is closely related to the positron-molecule complexes discussed here (Desfrançois et al., 1994, 2004; Abdoul-Carime and Desfrançois, 1998; Abdoul-Carime et al., 2002). In these "dipole-bound anions," the electron is only weakly bound to the target by a combination of dipolar, quadrapolar, and polarization forces (Abdoul-Carime and Desfrançois, 1998; Gutowski et al., 1998). Because of the small binding energy, the wave function of the excess electron is diffuse, residing predominantly outside the molecule due to the Pauli exclu- 
TABLE VIII. Measured and calculated electron binding energies and positron binding energies (in $\mathrm{meV})$.

\begin{tabular}{|c|c|c|c|c|c|c|c|}
\hline \multirow[b]{2}{*}{ Molecule } & \multirow[b]{2}{*}{ Formula } & \multirow{2}{*}{$\begin{array}{l}\mu^{\mathrm{a}} \\
\text { (D) }\end{array}$} & \multirow{2}{*}{$\begin{array}{c}\alpha_{d}{ }^{\mathrm{a}} \\
\left(\AA^{3}\right)\end{array}$} & \multicolumn{2}{|c|}{ Electron } & \multicolumn{2}{|c|}{ Positron } \\
\hline & & & & $\varepsilon_{b}{ }^{\mathrm{b}}$ & $\varepsilon_{b}{ }^{\mathrm{c}}$ & $\varepsilon_{b}^{\mathrm{d}}$ & $\varepsilon_{b}{ }^{\mathrm{e}}$ \\
\hline Formaldehyde & $\mathrm{CH}_{2} \mathrm{O}$ & 2.33 & 2.8 & & $0.02-0.05$ & 18 & 12 \\
\hline Acetaldehyde & $\mathrm{CH}_{3} \mathrm{CHO}$ & 2.75 & 4.6 & 0.65 & 0.95 & & 42 \\
\hline Acetone & $\left(\mathrm{CH}_{3}\right)_{2} \mathrm{CO}$ & 2.88 & 6.4 & 2.8 & 1.6 & 4 & 66 \\
\hline Hydrogen cyanide & $\mathrm{HCN}$ & 2.98 & 2.6 & & $3.3-5$ & 35 & 21 \\
\hline Nitrobenzene & $\mathrm{C}_{6} \mathrm{H}_{5} \mathrm{NO}_{2}$ & 4.2 & 13.5 & 28 & 30.4 & & 270 \\
\hline
\end{tabular}

${ }^{\mathrm{a}}$ Values used by Abdoul-Carime and Desfrançois (1998).

${ }^{\mathrm{b}}$ Experimental data (Abdoul-Carime and Desfrançois, 1998).

${ }^{c}$ Electrostatic model calculations (Abdoul-Carime and Desfrançois, 1998).

${ }^{\mathrm{d}}$ Calculated values, see Table VII.

${ }^{\mathrm{e}}$ Predictions of Eq. (61).

sion principle. In the positron-molecule case, the analogous short-range repulsion is due to the positive charges of atomic cores. The minimum dipole moment required for the formation of stable anions of common closedshell molecules has been determined experimentally to be 2-2.5 D (Desfrançois et al., 1994), in qualitative agreement with the critical dipole moment of $3.6 \mathrm{D}$, predicted by Eq. (61).

Table VIII lists the measured and calculated binding energies and relevant molecular parameters for a selection of these dipole-bound anions (Abdoul-Carime and Desfrançois, 1998). This table also lists the positron binding energies from quantum-chemistry calculations (where available) and estimated from Eq. (61). Positron binding energies are generally greater than their electron counterparts. This could be related to an electronpositron correlation effect known as virtual Ps formation, which contributes significantly to the positron-atom attraction (Dzuba et al., 1995; Gribakin and Ludlow, 2004). One consequence of this is that positrons are predicted to bind to atoms such as $\mathrm{Mg}, \mathrm{Zn}$, and $\mathrm{Cd}$, which do not form stable negative ions (Dzuba et al., 1995; Mitroy et al., 2002).

Another interesting electron analog of positronmolecule bound states is the case of negative ions of small molecular clusters, such as $\left(\mathrm{N}_{2} \mathrm{O}\right)_{n}$ (Weber et al., 1999) and $\left(\mathrm{CO}_{2}\right)_{n}$ (Leber et al., 2000). Using laserassisted photoelectron attachment, Klar et al. (1992a, 1992b, 1994) observed prominent VFRs in the yields of fragment negative ions [i.e., $\left(\mathrm{N}_{2} \mathrm{O}\right)_{q} \mathrm{O}^{-}$with $q<n$ and $\left(\mathrm{CO}_{2}\right)_{q}{ }^{-}$with $\left.q \leqslant n\right]$. These experiments are particularly relevant to the resonant processes discussed in this review. First, these VFRs were clearly identified with individual molecular vibrational modes. Second, these resonances displayed downshifts with increasing cluster size (especially clear for the $\mathrm{CO}_{2}$ clusters), which provided a measure of the electron-cluster binding energies. Finally, these cluster anions have the structure of a weakly bound, diffuse excess electron attached to an essentially unperturbed neutral cluster, similar to the positronmolecule bound states described here.
For the $\mathrm{CO}_{2}$ clusters, the measured electron binding energies are comparable to the positron $\varepsilon_{b}$ for alkanes with similar numbers of carbon atoms. They also increase approximately linearly for $n=4-20$, with some evidence of saturation at larger $n$. This behavior has been successfully modeled theoretically by combining the $-\alpha_{d} / 2 r^{4}$ polarization outside the cluster with a constant short-range potential inside (Leber et al., 2000). It is likely that including the long-range $-\alpha_{d} / 2 r^{4}$ potential would also improve the modeling of positron binding to alkanes (cf. Fig. 9).

A theory has been constructed to describe electron collisions with van der Waals clusters, such as $\left(\mathrm{CO}_{2}\right)_{n}$ (Fabrikant, 2005; Fabrikant and Hotop, 2005). It allows one to calculate the attachment cross sections and describe the VFR that are observed. In this theory the electron interaction with vibrational degrees of freedom of the $\mathrm{CO}_{2}$ monomers is described in the dipole approximation. This is analogous to the approach used in Sec. II.F to describe positron-molecule VFR.

\section{ANALYSIS OF ANNIHILATION RATES MEASURED WITH THERMALIZED POSITRONS}

There is an extensive body of experimental data on the annihilation of thermalized positrons in molecular gases at $300 \mathrm{~K}$ (Paul and Saint-Pierre, 1963; Heyland et al., 1982; Surko, Passner, et al., 1988; Iwata et al., 1995). It is useful to examine these results in light of the more recent, energy-resolved data for $Z_{\text {eff }}$ described above (Barnes et al., 2003, 2006) and the current understanding of resonant annihilation. In particular, it is now possible to consider the link between the thermal $Z_{\text {eff }}$ values, VFR and IVR. Figure 24 shows a comparison of energyresolved $Z_{\text {eff }}$ spectra for alkanes with $n=3-8$ carbons. For each molecule, its $Z_{\text {eff }}$ was normalized by $g=\sqrt{\varepsilon_{b} / \varepsilon}$, shifted upward in energy by the binding energy $\varepsilon_{b}$, and then normalized to unity at the $\mathrm{C}-\mathrm{H}$ stretch peak. In this representation, it is clear that the spectral shapes of the alkanes change relatively little with molecular size. As 
the size of an alkane increases, its $Z_{\text {eff }}$ spectrum shifts to lower energies. A mode accessed at $50 \mathrm{meV}$ incident positron energy in the butane $(n=4)$ spectrum will be accessed at $5 \mathrm{meV}$ in hexane $(n=6)$. Thus, the annihilation rates $Z_{\mathrm{eff}}^{\mathrm{th}}$ measured with thermalized positrons at $300 \mathrm{~K}$ are intimately related to the corresponding values of the energy-resolved spectra, albeit shifted downward by the binding energy.

As shown in Fig. 24, the $300 \mathrm{~K}$ data (plus signs in circles) align well with the energy-resolved data when they are normalized by $g=\sqrt{\varepsilon_{b} / \varepsilon_{T}}$ (and the C-H stretch peak heights) and assigned a corrected energy of $\varepsilon_{b} / \varepsilon_{T}$, where $\varepsilon_{T}=\frac{3}{2} k_{B} T=37.5 \mathrm{meV}$ is the average thermal positron energy at $300 \mathrm{~K}$. Also note that, due to their larger binding energies, alkanes with nine or more carbons begin to sample the trough in the spectrum between the plateau and the $\mathrm{C}-\mathrm{H}$ stretch peaks. This explains the trend, pointed out previously (Barnes et al., 2003), that the ratio of $Z_{\text {eff }}^{\text {th }}$ to $Z_{\text {eff }}$ at the C-H stretch peak decreases by a factor of 2 when the number of carbon atoms in the alkane is increased from $n \leqslant 9$ to $n>9$. This analysis connects in a quantitative way the thermal data with the energy-resolved measurements taken at higher positron energies. Thermal positrons at $300 \mathrm{~K}$ annihilate by the same IVR-enhanced resonant mechanism as higherenergy positrons; the only difference is the specific vibrational resonances involved.

The values of $Z_{\text {eff }}^{\text {th }}$ measured at $300 \mathrm{~K}$ can also be used to test Eq. (61) which estimates the positron binding energy in terms of molecular parameters (Danielson et al., 2009). A selection of molecules and their thermal annihilation rates $Z_{\text {eff }}^{\text {th }}$ from Danielson et al. (2009) are listed in Table IX. While these $Z_{\text {eff }}^{\text {th }}$ values do not provide direct evidence of VFR and hence positron binding, they can indicate whether positrons do or do not bind to the molecular species (Gribakin, 2000, 2001; Iwata et al., 2000; Young and Surko, 2008b). We take $Z_{\mathrm{eff}}^{\mathrm{th}}>10^{3}$ (Sec. II.D) to be an indicator of resonant annihilation and hence positron binding. This is by no means a necessary condition, and so in the analysis below, we also use the somewhat arbitrary condition, $Z_{\mathrm{eff}}^{\mathrm{th}} / Z=10$, as the boundary separating the two groups of molecules. Namely, $Z_{\mathrm{eff}}^{\mathrm{th}} / Z>10$ for a given molecule is taken to mean that positrons bind to this target and vice versa. The predictions of Eq. (61) are listed in Table IX, together with the molecular parameters used to calculate them. Generally, molecules with relatively large values of $Z_{\mathrm{eff}}^{\text {th }} / Z$ are predicted correctly by Eq. (61) to have positive binding energies. One borderline exception is methane which has a value of $Z_{\text {eff }}^{\text {th }} / Z=14$ but does not bind positrons.

As shown in Table IX, the criterion from Eq. (61) that molecules will bind for $\alpha_{d}>5.4 \AA^{3}$ has the consequence that most large molecules can bind positrons whether they have a permanent dipole moment or not. The alkanes are an example of this, where only methane does not bind. For nonaromatic molecules the $\alpha_{d}$ term dominates for all but relatively small molecules with correspondingly small values of $\alpha_{d}$. A related trend is seen in halogen substitution, where $\varepsilon_{b}$ rises rapidly as the size (and hence the polarizability) of the halogen is increased. For example, $\mathrm{CF}_{4}$ does not bind positrons, while $\mathrm{CBr}_{4}$ is predicted to have a binding energy in excess of $120 \mathrm{meV}$.

Restricting comparison to cases where the model predicts $\left|\varepsilon_{b}\right| \geqslant 15 \mathrm{meV}$ in deference to the likely error bars for the model, negative binding energies are predicted for almost all small molecules. In particular, the diatomic molecules, $\mathrm{H}_{2}, \mathrm{D}_{2}, \mathrm{~N}_{2}, \mathrm{O}_{2}, \mathrm{CO}$, and $\mathrm{NO}$ (not shown in Table IX), with $Z_{\text {eff }}^{\text {th }} / Z$ values ranging from 2.3 to 7.4 (i.e., $<10$ ) all have negative binding energies $\varepsilon_{b}$ $<-40 \mathrm{meV}$ (Danielson et al., 2009). As shown in Table IX, neither methane nor carbon tetrafluoride bind, in agreement with the interpretation of their $Z_{\text {eff }}^{\text {th }}$ values (Iwata et al., 1995). As indicated in Table VI, molecules for which the binding energies are "too close to call," [i.e., $\left|\varepsilon_{b}\right|<15 \mathrm{meV}$, based on Eq. (61)] include ethane, propane, cyclopropane, methanol, methyl fluoride, and water (cf. Table IX), and this is consistent with the observations (Young and Surko, 2008c). There are some disagreements in cases where the binding energy is predicted and/or observed to be reasonably small. From Table IX, the only molecule that exceeds the $\left|\varepsilon_{b}\right|$ $\geqslant 15 \mathrm{meV}$ criterion is $\mathrm{NO}_{2}$ with a $Z_{\mathrm{eff}}^{\text {th }} / Z$ ratio of 47 and yet a predicted binding energy of $-26 \mathrm{meV}$. Like benzene, $\mathrm{NO}_{2}$ has two resonant electronic states involving $\pi$ bonds, but the geometry of its valence orbitals differs greatly from that of the aromatics. Additional experiments are needed to better distinguish the effects of the different types of electronic bonds, especially for the smallest molecules.

As shown in Table IX, the ratios of $Z_{\text {eff }}^{\text {th }} / Z$ for perfluoroalkane molecules are much smaller than those for alkanes. This has been interpreted as evidence that resonant annihilation is "switched off" for them, possibly due to lack of binding (Gribakin, 2000). However, Eq. (61) predicts that perfluorocarbons other than perfluoromethane and perfluoroethane can bind positrons. This warrants further investigation.

\section{OTHER TOPICS}

\section{A. Gamma-ray Doppler-broadening measurements}

As discussed in Sec. II.B, positron annihilation usually results in the production of two, approximately back-toback gamma rays, each with an energy $E_{\gamma} \approx 511 \mathrm{keV}$. However, there are small shifts in the energies of the two gammas that can be used to obtain microscopic information about the annihilating pair. According to Eq. (13), these energies are Doppler shifted by the energy $\varepsilon= \pm c P_{z} / 2$, where $P_{z}$ is the component of the center-ofmass momentum of the electron-positron pair along the direction of the gamma rays. For the low-energy positrons that we deal with here (i.e., $\varepsilon \leqslant 0.5 \mathrm{eV}$ ), the centerof-mass momentum $P$ is dominated by the momentum distribution of the electron orbitals. As a benchmark, a $4 \mathrm{eV}$ electron traveling along the direction of the 
TABLE IX. Values of $Z_{\mathrm{eff}}^{\mathrm{th}} / Z$ for a variety of chemical species, the predictions of Eq. (61) for their binding energies, the molecular parameters relevant to this analysis, and the molecular ionization energies $E_{i}$.

\begin{tabular}{|c|c|c|c|c|c|c|c|}
\hline Name & Formula & $\begin{array}{c}\varepsilon_{b}{ }^{\mathrm{a}} \\
(\mathrm{meV})\end{array}$ & $Z$ & $Z_{\mathrm{eff}}^{\mathrm{th}} / Z^{\mathrm{b}}$ & $\begin{array}{c}\alpha_{d}^{\mathrm{c}} \\
\left(\AA^{3}\right)\end{array}$ & $\begin{array}{l}\mu^{\mathrm{c}} \\
(\mathrm{D})\end{array}$ & $\begin{array}{c}E_{i}^{\mathrm{c}} \\
(\mathrm{eV})\end{array}$ \\
\hline \multicolumn{8}{|c|}{ Small molecules } \\
\hline Carbon dioxide & $\mathrm{CO}_{2}$ & -36 & 22 & 2.5 & 2.7 & 0 & 13.8 \\
\hline Sulfur hexafluoride & $\mathrm{SF}_{6}$ & -14 & 70 & 1.2 & 4.5 & 0 & 15.3 \\
\hline Water & $\mathrm{H}_{2} \mathrm{O}$ & -15 & 10 & 32 & 1.5 & 1.9 & 12.6 \\
\hline Nitrous oxide & $\mathrm{N}_{2} \mathrm{O}$ & -29 & 22 & 3.5 & 3.0 & 0.2 & 12.9 \\
\hline Nitrogen dioxide & $\mathrm{NO}_{2}$ & -26 & 23 & 47 & 3.0 & 0.3 & 9.8 \\
\hline Ammonia & $\mathrm{NH}_{3}$ & -12 & 10 & 160 & 2.3 & 1.5 & 10.2 \\
\hline Methane & $\mathrm{CH}_{4}$ & -37 & 10 & 14 & 2.6 & 0 & 12.7 \\
\hline \multicolumn{8}{|c|}{ Alkenes and alkynes } \\
\hline 1-hexene & $\mathrm{C}_{6} \mathrm{H}_{12}$ & 81 & 48 & 3900 & 11.6 & 0.3 & 9.5 \\
\hline trans 3-hexene & $\mathrm{C}_{6} \mathrm{H}_{12}$ & 74 & 48 & 4100 & 11.6 & 0 & 8.9 \\
\hline 1,3-hexadiene & $\mathrm{C}_{6} \mathrm{H}_{10}$ & $72^{\mathrm{d}}$ & 46 & 8500 & 11.4 & & 8.5 \\
\hline 1,3,5-hexatriene & $\mathrm{C}_{6} \mathrm{H}_{8}$ & 69 & 44 & 9400 & 11.2 & 0 & 8.3 \\
\hline \multicolumn{8}{|c|}{ Perhalogenated alkanes } \\
\hline Carbon tetrafluoride & $\mathrm{CF}_{4}$ & -34 & 42 & 1.2 & 2.9 & 0 & 16.2 \\
\hline Hexafluoroethane & $\mathrm{C}_{2} \mathrm{~F}_{6}$ & -10 & 66 & 2.3 & 4.8 & 0 & 14.6 \\
\hline Perfluoropropane & $\mathrm{C}_{3} \mathrm{~F}_{8}$ & $13^{\mathrm{d}}$ & 90 & 1.7 & 6.7 & & \\
\hline Perfluorohexane & $\mathrm{C}_{6} \mathrm{~F}_{14}$ & 84 & 162 & 3.3 & 12.4 & 0 & 12.8 \\
\hline Perfluorooctane & $\mathrm{C}_{8} \mathrm{~F}_{18}$ & 131 & 210 & 5.1 & 16.2 & 0 & 12.6 \\
\hline Carbon tetrachloride & $\mathrm{CCl}_{4}$ & 58 & 74 & 130 & 10.3 & 0 & 11.3 \\
\hline Carbon tetrabromide & $\mathrm{CBr}_{4}$ & 120 & 146 & 270 & 15.3 & 0 & 10.3 \\
\hline Carbon tetraiodide & $\mathrm{CI}_{4}$ & 235 & 218 & 37 & 24.5 & 0 & \\
\hline \multicolumn{8}{|c|}{ Partially fluorinated alkanes } \\
\hline Difluoromethane & $\mathrm{CH}_{2} \mathrm{~F}_{2}$ & 0.4 & 26 & 31 & 2.5 & 1.8 & 12.6 \\
\hline Trifluoromethane & $\mathrm{CHF}_{3}$ & -4 & 34 & 7.3 & 2.7 & 1.7 & 14.8 \\
\hline Fluoroethane & $\mathrm{C}_{2} \mathrm{H}_{5} \mathrm{~F}$ & 21 & 26 & 120 & 4.2 & 2.0 & 12.4 \\
\hline 1,1,1-trifluoroethane & $\mathrm{C}_{2} \mathrm{H}_{3} \mathrm{~F}_{3}$ & 29 & 42 & 38 & 4.2 & 2.3 & 13.3 \\
\hline \multicolumn{8}{|c|}{ Oxygen-containing molecules } \\
\hline 1-propanol & $\mathrm{C}_{3} \mathrm{H}_{8} \mathrm{O}$ & 50 & 34 & 590 & 7.0 & 1.7 & 10.2 \\
\hline Acetone & $\mathrm{C}_{3} \mathrm{H}_{6} \mathrm{O}$ & 66 & 32 & 3100 & 6.3 & 2.9 & 9.7 \\
\hline \multicolumn{8}{|c|}{ Other aromatics and substituted benzenes } \\
\hline Anthracene & $\mathrm{C}_{14} \mathrm{H}_{10}$ & 422 & 94 & 46000 & 22.8 & 0 & 7.5 \\
\hline Decahydronaphthalene & $\mathrm{C}_{10} \mathrm{H}_{18}$ & 151 & 78 & 5000 & 17.7 & 0 & 9.4 \\
\hline o-xylene & $\mathrm{C}_{8} \mathrm{H}_{10}$ & 208 & 58 & 3100 & 14.1 & 0.6 & 8.6 \\
\hline Toluene & $\mathrm{C}_{7} \mathrm{H}_{8}$ & 179 & 50 & 3800 & 12.3 & 0.4 & 8.8 \\
\hline Hexafluorobenzene & $\mathrm{C}_{6} \mathrm{~F}_{6}$ & 141 & 90 & 13 & 9.8 & 0 & 9.9 \\
\hline Octafluorotoluene & $\mathrm{C}_{7} \mathrm{~F}_{8}$ & $165^{\mathrm{d}}$ & 114 & 11 & 11.7 & & 9.9 \\
\hline Octafluoronaphthalene & $\mathrm{C}_{10} \mathrm{~F}_{8}$ & 272 & 132 & 23 & 15.5 & 0 & 8.9 \\
\hline Nitrobenzene & $\mathrm{C}_{6} \mathrm{H}_{5} \mathrm{NO}_{2}$ & 254 & 64 & 6700 & 12.1 & 4.2 & 9.9 \\
\hline Chlorobenzene & $\mathrm{C}_{6} \mathrm{H}_{5} \mathrm{Cl}$ & 204 & 58 & 1250 & 12.1 & 1.6 & 9.1 \\
\hline Bromobenzene & $\mathrm{C}_{6} \mathrm{H}_{5} \mathrm{Br}$ & 215 & 76 & 2300 & 13 & 1.7 & 9.0 \\
\hline Fluorobenzene & $\mathrm{C}_{6} \mathrm{H}_{5} \mathrm{~F}$ & 176 & 50 & 900 & 10.0 & 1.6 & 9.2 \\
\hline 1,2-difluorobenzene & $\mathrm{C}_{6} \mathrm{H}_{4} \mathrm{~F}_{2}$ & 189 & 58 & 570 & 9.8 & 2.4 & 9.3 \\
\hline
\end{tabular}

${ }^{\mathrm{a}}$ Predicted by Eq. (61).

${ }^{\mathrm{b}} Z_{\mathrm{eff}}^{\mathrm{th}}$ is measured at room temperature with thermalized positrons (Iwata et al., 1995; Iwata, 1997).

${ }^{c}$ Parameters from McClellan (1963), Miller (1990), and Lide (2000).

${ }^{\mathrm{d}}$ Lacking values of $\mu$ for these species, the predictions of Eq. (61) are lower bounds on $\varepsilon_{b}$. 


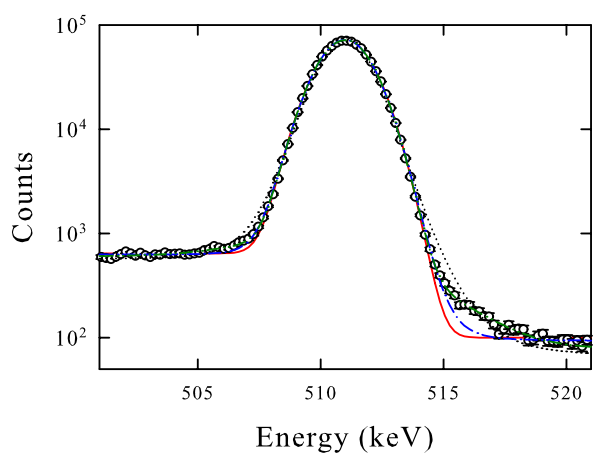

FIG. 35. (Color online) $\gamma$-ray spectrum of hexane $\left(\mathrm{C}_{6} \mathrm{H}_{14}\right):(\bigcirc)$ observed spectrum; solid curve, single Gaussian fit; dotted curve, fit using a hydrogenic functional form; dot-dashed curve, fit using a noninteracting hydrogenic form convolved with a Gaussian; dashed curve (indistinguishable from the data), two Gaussian fit. The statistical error bars are comparable to or smaller than the size of the data points. See Iwata, Greaves, and Surko (1997) for details.

gamma rays produces a $1 \mathrm{keV}$ Doppler shift.

Extensive measurements have been made of the Doppler broadening of annihilation gamma rays for the case of a thermal distribution of positrons at $300 \mathrm{~K}$ interacting with a wide variety of molecules (Tang et al., 1992; Iwata, Greaves, and Surko, 1997). The apparatus and procedures for these measurements are described in Sec. III.F. Shown in Fig. 35 is the spectrum for hexane. The detector response is modeled by a Gaussian line shape with a FWHM of $1.16 \mathrm{keV}$ and an error function to account for the effect of Compton scattering of the gamma rays in the detector. The measured gamma spectra could be fit reasonably well using a single Gaussian to approximate the Doppler spectrum, convolved with the detector response function.

A more accurate fit was obtained using two Gaussians to model the Doppler spectrum. The second Gaussian was required to describe a smaller higher-momentum component, which was typically about $1-3 \%$ of the larger component. The resulting linewidths (FWHM), with the detector resolution deconvolved, ranged from $1.7 \mathrm{keV}$ for $\mathrm{H}_{2}$ to $\sim 3.1 \mathrm{keV}$ for fluorocarbons. The smaller higher-momentum component had widths from 4 to $9 \mathrm{keV}$, with most molecules in the narrower range from 5 to $7 \mathrm{keV}$.

Shown in Table $\mathrm{X}$ are typical values of the linewidths $\Delta E$ (FWHM) using the single Gaussian fit, the annihilation rates $Z_{\text {eff }}$ for thermal positrons, and the positronmolecule binding energies $\varepsilon_{b}$ for a selection of molecules. A key conclusion of these Doppler-broadening studies is that the linewidths are only weakly correlated with the values of either $\varepsilon_{b}$ or $Z_{\mathrm{eff}}^{\mathrm{th}}$. For example, comparing the ethane and dodecane in Table $\mathrm{X}, \varepsilon_{b}$ increases from near zero to $220 \mathrm{meV}$, and $Z_{\mathrm{eff}}^{\text {th }}$ increases by three orders of magnitude, while the gamma-ray linewidth increases by only $5 \%$. Note that there is, however, a significant increase in the linewidth when a hydrocarbon is partially or fully fluorinated.
TABLE X. Gamma-ray linewidths $\Delta E$ (FWHM, single Gaussian fit), annihilation rates $Z_{\text {eff }}^{\text {th }}$ for a thermal distribution of positrons at $300 \mathrm{~K}$, and positron-molecule binding energies $\varepsilon_{b}$ for selected molecules.

\begin{tabular}{lccrr}
\hline \hline Name & Formula & $\begin{array}{c}\Delta E^{\mathrm{a}} \\
(\mathrm{keV})\end{array}$ & \multicolumn{1}{c}{$Z_{\text {eff }}^{\text {th b }}$} & $\begin{array}{r}\varepsilon_{b}^{\mathrm{c}} \\
(\mathrm{meV})\end{array}$ \\
\hline Methane & $\mathrm{CH}_{4}$ & 2.09 & 142 & $<0$ \\
Ethane & $\mathrm{C}_{2} \mathrm{H}_{6}$ & 2.18 & 1780 & $>0$ \\
Propane & $\mathrm{C}_{3} \mathrm{H}_{8}$ & 2.21 & 3500 & 10 \\
Pentane & $\mathrm{C}_{5} \mathrm{H}_{12}$ & 2.24 & 40200 & 60 \\
Nonane & $\mathrm{C}_{7} \mathrm{H}_{16}$ & 2.32 & 643000 & 145 \\
Dodecane & $\mathrm{C}_{12} \mathrm{H}_{26}$ & 2.29 & 1780000 & 220 \\
Benzene & $\mathrm{C}_{6} \mathrm{H}_{6}$ & 2.23 & 15000 & 150 \\
Naphthalene & $\mathrm{C}_{10} \mathrm{H}_{8}$ & 2.29 & 494000 & $\sim 300$ \\
1-fluoroethane & $\mathrm{C}_{2} \mathrm{H}_{5} \mathrm{~F}$ & 2.62 & 3030 & $>0$ \\
Hexafluoroethane & $\mathrm{C}_{2} \mathrm{~F}_{6}$ & 3.04 & 149 & $<0$ \\
1-fluorohexane & $\mathrm{C}_{6} \mathrm{H}_{13} \mathrm{~F}$ & 2.46 & 269000 & 80 \\
\hline \hline
\end{tabular}

${ }^{\mathrm{a}}$ From Iwata, Greaves, and Surko, (1997).

${ }^{\text {b}}$ See Iwata et al. (1995), Iwata (1997), and references therein.

${ }^{\mathrm{c}}$ See Young and Surko $(2008 \mathrm{~b}, 2008 \mathrm{c})$ and Sec. VI.

The linewidths $\Delta E$ for alkanes, shown in Table $\mathrm{X}$, increase monotonically with increasing molecular size. This increase has been ascribed to the change in the relative number of electrons in $\mathrm{C}-\mathrm{C}$ vs $\mathrm{C}-\mathrm{H}$ bonds since the electrons in these bonds are expected to be characterized by different Doppler widths. An analysis was made to determine the fraction of annihilation events involving electrons from these types of orbitals in alkanes (Iwata, Greaves, and Surko, 1997). It showed that there is a linear increase in the linewidth as a function of the fraction of electrons in $\mathrm{C}-\mathrm{C}$ orbitals. This is consistent with the assumption that the positron annihilates statistically on any of the valence electrons in the molecule (see below).

A similar study was carried out for the linewidths associated with annihilation in partially and fully fluorinated alkanes. In this case, the observed line was decomposed into fluorinated and hydrogenated components by fitting to a sum of two measured line shapes, one for the fully hydrogenated compound and one for the fully fluorinated compound (Iwata, Greaves, and Surko, 1997). As shown in Fig. 36, there is a smooth, linear increase in the linewidth over the full range of the degree of fluorination, from 0 to $100 \%$. This also supports the hypothesis that the positrons annihilate statistically on any valence electron.

The results of these gamma-ray studies provide confirmation of a model by Crawford (1994) for positron annihilation on molecules. It was developed to explain the degree of ion fragmentation that is observed following positron annihilation on molecules (see Sec. VIII.B). Crawford argued that a low-energy positron should annihilate statistically on any valence electron. The gamma-ray linewidth measurements shown in Fig. 36 are consistent with this hypothesis. Such uniform 


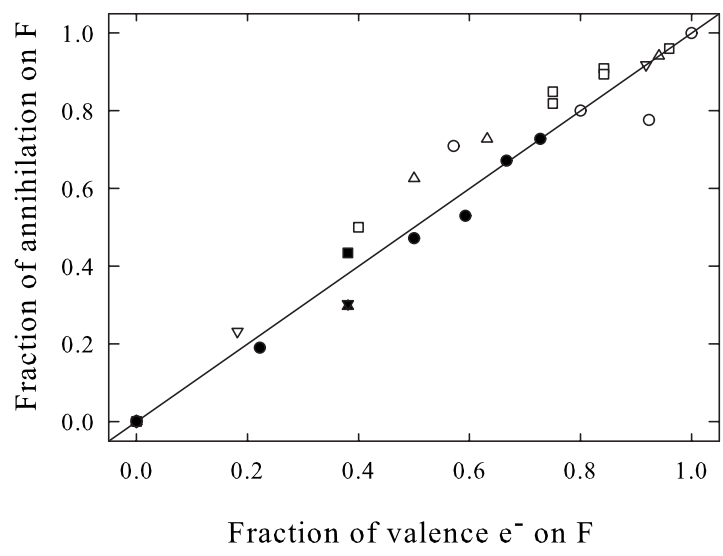

FIG. 36. Normalized fraction of positrons annihilating on fluorine atoms in partially and fully fluorinated alkanes, plotted against the fraction of valence electrons in the fluorine atoms. Open symbols are methane (circle), ethane (square), propane (triangle), and hexane-based molecules (inverted triangle); filled symbols are six-carbon benzene-based molecules: 1,2difluorobenzene (square), 1,3-difluorobenzene (triangle), 1,4difluorobenzene (inverted triangle), and other six-carbon benzene-based molecules (circle). See text and Iwata, Greaves, and Surko (1997) for details.

spreading of the positron density over the molecule is related to the fact that at low energies the positron de Broglie wavelength $\lambda_{\mathrm{deB}}$ is larger than the size of the target. This prevents the positron from being localized strongly on any particular site in the molecule. This is true both for direct annihilation, for which $\lambda_{\mathrm{deB}}=2 \pi / k$ (e.g., $k=0.05$ a.u. for thermal positrons at $300 \mathrm{~K}$ ), and for resonant annihilation, for which $\lambda_{\mathrm{deB}} \sim 2 \pi / \kappa$, where $\kappa=\sqrt{2 \varepsilon_{b}}$ (see, e.g., Fig. 10 for model bound-state wave functions).

In some cases, such as strongly polar molecules, localization of the positron near specific sites in the molecule might be expected. For example, in $\mathrm{LiH}$ the positron density is strongly localized at the negatively charged hydrogenic end of the molecule (Strasburger, 1999, 2001); cf. Fig. 8. However, annihilation gamma-ray spectra from this class of molecules have not yet been investigated.

An analysis is in progress to understand in more detail the implications of the Doppler-broadening experiments (Wang et al., 2010). It relies on modern quantumchemistry methods, such as the density-functional theory B3LYP/TZVP, which give electron momentum densities in good agreement with experiment (Wang, 2003). The linewidths calculated for methane and fluoromethanes, ethane, propane, butane, and benzene, by taking only the electron momentum distribution into account, are about $30 \%$ greater than the values measured. The main source of this discrepancy appears to be the neglect of the Coulomb repulsion between the positron and the atomic cores (Wang et al., 2010). This repulsion suppresses the positron wave function at small positronnuclear separations, which effectively reduces the highDoppler-shift components in the annihilation spectra.
A more complete theory of the annihilation gammaray spectra for molecules should include the full electron and positron dynamics and account for the positronnuclear repulsion and electron-positron correlation effects. However, the results of Wang et al. (2010) suggest that these effects in positron annihilation spectra can be modeled by a relatively simple scaling factor. At the next level of analysis, one should examine the highmomentum component in the spectra [e.g., as described by two-Gaussian fits (Iwata et al., 1997)], which is likely due to annihilation on inner-shell electrons. Further work on this topic is in progress.

\section{B. Annihilation-induced fragmentation of molecules}

Two-body collisions between positrons and molecules can produce positive ions by two mechanisms. For incident positron energies greater than the threshold for Ps formation, ionization proceeds via this channel. If the positron energy is below the Ps formation threshold (i.e., the principal regime of interest in this review), the positron can annihilate with a molecular electron, also producing a positive molecular ion.

The initial studies of annihilation-induced ion formation in molecules below the positronium formation threshold were conducted using positrons confined in a buffer-gas trap in the presence of low pressure gases of alkane molecules (Passner et al., 1989). In spite of complications due to the presence of molecular nitrogen in the trap (Glish et al., 1994), these experiments established that sub-Ps-threshold ionization can produce significant amounts of fragmentation. Subsequently, extensive studies of positron-induced ionization of molecules, both below and above the positronium formation threshold, were conducted by Hulett and collaborators (Donohue et al., 1990; Hulett et al., 1993; Xu et al., 1993, 1994, 1995, 1997; Hulett, Xu, McLuckey, Lewis, et al., 1996; Hulett, Xu, McLuckey, and Schrader, 1996; Moxom et al., 2000). The experimental procedures are described in Sec. III.G.

These studies produced several important results. One is that the extent of fragmentation depends in a nontrivial way on the energy of the incident positron. For example, fragmentation is a minimum at energies near the Ps-formation threshold, and increases toward smaller and greater positron energies. Figure 37 shows the mass spectrum of 1-dodecene $\left(\mathrm{C}_{12} \mathrm{H}_{24}\right)$ at an incident energy of $1.0 \mathrm{eV}$ (i.e., $\sim 2 \mathrm{eV}$ below the Ps formation threshold). It illustrates the broad spectrum of product ions that is produced (Xu et al., 1994). Figure 38 shows the cross sections for producing these fragments as a function of positron energy.

The fact that in many molecules the degree of fragmentation has a minimum close to the Ps-formation threshold may offer the possibility of using positrons to advantage in ion mass spectroscopy. Another interesting effect is that, below the Ps-formation threshold, double and triple bonds can stabilize the species with respect to fragmentation. For example, in the series decane $\left(\mathrm{C}_{10} \mathrm{H}_{22}\right.$, single bonds only), 1-decene $\left(\mathrm{C}_{10} \mathrm{H}_{20}\right.$, one 


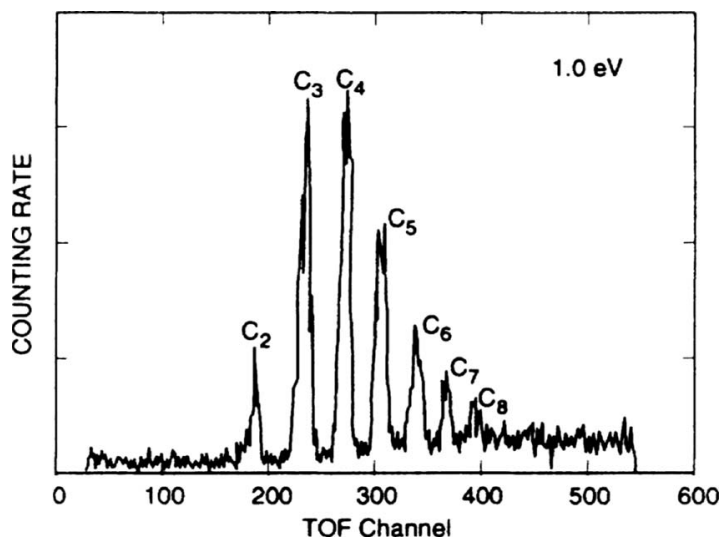

FIG. 37. Time-of-flight mass spectra of ion fragments from 1-dodecene at $1.0 \mathrm{eV}$, which is $2.1 \mathrm{eV}$ below the Ps formation threshold. Adapted from Xu et al., 1994.

double bond) and 1,9-decadiene $\left(\mathrm{C}_{10} \mathrm{H}_{18}\right.$, two double bonds), the yield of ion fragments decreases with each additional double bond (Xu et al., 1995). This same effect was also observed in other molecules [e.g., tetravinylsilane vs tetraethylsilane (Hulett et al., 1993; Xu et al., 1995)].

Motivated by these experiments, Crawford (1994) constructed a simple and insightful model of the fragmentation process. He argued that annihilation will occur with comparable probability on electrons in any valence molecular orbital $i$, not just the highest occupied molecular orbital (HOMO). As a result, the molecular ion is typically left in an electronically excited state, with the excitation energy provided by the energy difference between the HOMO and the orbital $i$. Figure 39 shows the probability that this energy exceeds a certain value for propane, hexane, and decane (Crawford, 1994). Due to vibronic coupling, this energy flows from the electronic to the vibrational degrees of freedom. Given time and sufficient energy (e.g., a few electron volts), the mo-

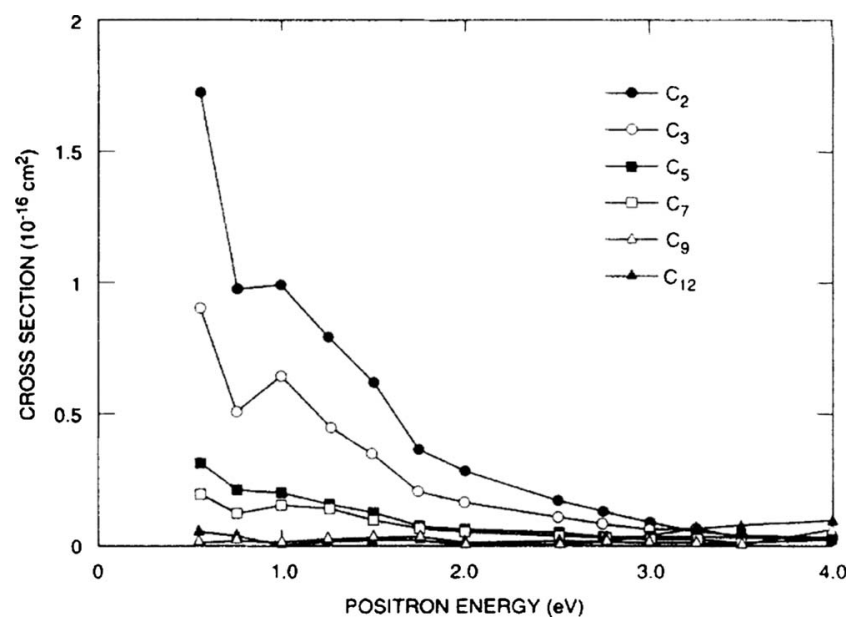

FIG. 38. Cross sections for fragmentation of 1-dodecene $\left(\mathrm{C}_{12} \mathrm{H}_{24}\right)$ by positron annihilation near and below the Psformation threshold at $3.1 \mathrm{eV}$. From Xu et al., 1994.

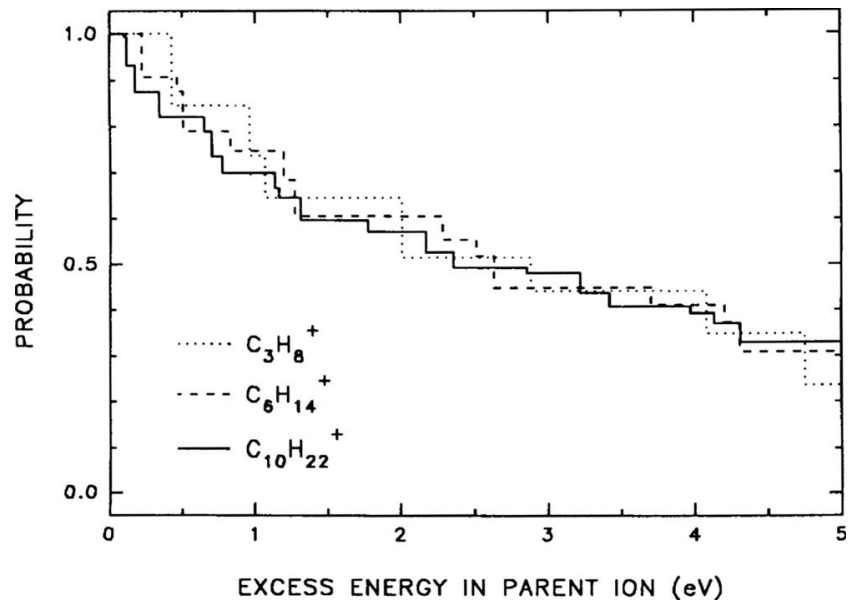

FIG. 39. Probability $P(E)$ that the energy deposited into a molecular ion due to positron annihilation with an electron in a valence orbital below the HOMO is greater than a given excess energy for propane, hexane, and decane. For details of the calculation, see Crawford (1994).

lecular ion can break up in the process known as unimolecular dissociation.

Fragmentation can occur when the incident positron annihilates, either during direct annihilation or following capture into a VFR. The resonant process likely dominates in larger polyatomics at low positron energies and is central to this review. In this case, both the incident positron energy and the binding energy contribute to the total excitation energy of the molecular ion. The gamma-ray annihilation studies described above provided validation of Crawford's model, showing that positrons do indeed annihilate with approximately equal probabilities on all valence electrons.

The experiments of Hulett and co-workers used a beam with an energy resolution $\sim 0.5 \mathrm{eV}$ FWHM. This raises a question as to the extent to which ionization could be controlled (e.g., to produce only parent ions) using the much higher resolution beams that are now available (e.g., $40 \mathrm{meV}$ FWHM). Another possibility would be to exploit ionization via the second bound states such as those observed in larger alkane molecules (cf. Sec. V.B). In this case, the positron wave function has a nodal plane at the center of the linear carbon chain (see Fig. 10), which would be expected to produce a decrease in annihilation near this location. An experiment to test this might also provide information as to whether the excess electronic energy deposited in the molecule in the annihilation process could diffuse away from the annihilation site and then break a bond. Investigations to date have only scratched the surface of this rich area of matter-antimatter chemistry. We still know relatively little about the chemical specificity of annihilation and annihilation-induced ion production.

\section{Nonlinear dependence of annihilation on molecular density}

Equations (2) and (10) imply that the annihilation rate $\lambda$ is a linear function of the gas density $n$. This was 


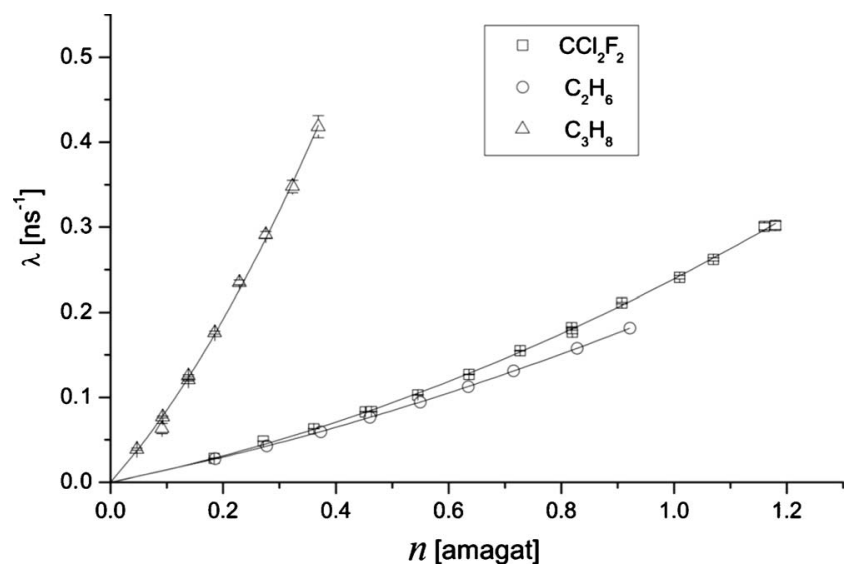

FIG. 40. Density dependence of the room-temperature positron annihilation rates for $\mathrm{CCl}_{2} \mathrm{~F}_{2}(\square), \mathrm{C}_{2} \mathrm{H}_{6}(\bigcirc)$, and $\mathrm{C}_{3} \mathrm{H}_{8}$ $(\triangle)$. Solid lines are fits of the form Eq. (62). Adapted from Charlton et al., 2006.

shown to be correct at low densities, such as those used in the positron-trap experiments described in Sec. III.C. There are, however, a number of effects that can make $Z_{\text {eff }}$ density dependent. For example, in dense gases and at lower temperatures, positrons can cause a local phase transition and become self-trapped in clusters that gives rise to a strongly nonlinear behavior of the annihilation rate (Iakubov and Khrapak, 1982). Also, in low-density gases one typically observes $Z_{\text {eff }} \gg Z$, while at solid or liquid densities the value of $Z_{\text {eff }}$ for almost any material are close to the number of valence electrons (Pomeranchuk, 1949).

For diatomic molecular gases such as $\mathrm{H}_{2}, \mathrm{~N}_{2}, \mathrm{CO}$, and $\mathrm{O}_{2}$ at densities $n \lesssim 50$ amagat, the $Z_{\text {eff }}$ values remain practically independent of $n$. In contrast, other species, such as $\mathrm{CO}_{2}, \mathrm{CH}_{4}$, and $\mathrm{SF}_{6}$, display considerable variation with $n$ (Heyland et al., 1982). For larger molecules (e.g., $\mathrm{C}_{2} \mathrm{H}_{6}, \mathrm{CCl}_{2} \mathrm{~F}_{2}, \mathrm{C}_{3} \mathrm{H}_{8}$, and $\mathrm{C}_{4} \mathrm{H}_{10}$ ) a strong density dependence is observed even at $n \sim 1$ amagat (Heyland et al., 1982), as shown in Fig. 40. In these cases, the density effect can be expressed as a quadratic correction to Eq. (10),

$$
\lambda=a n+b n^{2} .
$$

Here $a=\pi r_{0}^{2} c Z_{\text {eff }}$ represents annihilation in binary collisions, and the $b$ term accounts for three-body annihilation events involving a positron and two gas molecules (Charlton et al., 2002, 2006). A key finding is that the coefficient $b$ for molecules with large $Z_{\text {eff }}$, for which resonant annihilation is observed (e.g., $\mathrm{C}_{2} \mathrm{H}_{4}$ or $\mathrm{C}_{3} \mathrm{H}_{8}$ ), is approximately proportional to $Z_{\text {eff }}$. This suggests that three-body collisions affect the resonant annihilation rather than being an additional independent reaction pathway.

There is currently no information about the microscopic nature of this process, and so one can only mention some possibilities. The annihilation in the resonant positron-molecule complex could be enhanced through collisions with other molecules. Gribakin (2009) recently proposed that these collisions may stabilize the resonant complexes. If this stabilization is complete (i.e., preventing the positron from escaping the molecule), then the corresponding collision rate $\Gamma^{c}=\sigma_{c} v_{m} n$ must be added to the annihilation rate $\Gamma_{\nu}^{a}$ in the numerator of Eq. (37). Here $v_{m}$ is the mean molecular collision velocity and $\sigma_{c}$ is the stabilizing collision cross section. The values of $\sigma_{c}$ required to explain the observations can be estimated using the experimental values of $a$ and $b$ (Charlton et al., 2006) and estimating $\Gamma_{\nu}^{a}$ from Eqs. (23) and (36) using the binding energies inferred from the energy-resolved $Z_{\text {eff }}$ measurements. The values range from $\sigma_{c} \sim 1$ a.u. in ethane and ethylene to $\sim 10$ a.u. in butane.

There is also the possibility that a collision with a second molecule enhances the annihilation rate by an amount $p \Gamma^{c}$, where $p<1$ is the probability that the collisionally stabilized positron-molecule complex survives to annihilation rather than autodetaches. In this case, to achieve the same effect, the cross sections $\sigma_{c}$ would need to be enhanced by a factor $p^{-1}$ above that for complete stabilization.

The actual mechanism of collisional stabilization is also unclear. It could be due to the transfer of the bound positron from the vibrationally excited host molecule to a vibrationally colder molecule of the same species. This process would be facilitated by the fact that the positron binding energies in the two molecules would be similar, making it a resonant charge transfer. The vibrational deexcitation of the positron-molecule complex could also be due to intermolecular vibrational energy transfer in a collision with a colder molecule. However, in both cases, for the species studied, the thermal vibrational energies at room temperature are larger than the positron binding energies. Hence these processes might not completely stabilize the complex.

Finally, if a VFR is excited when two molecules are in close proximity, the positron binding energy is likely to be greater than that for a binary positron-molecule interaction. This too might help to explain the observations. At a minimum, further research is warranted to understand this collisional enhancement effect.

\section{SUMMARY AND A LOOK TO THE FUTURE}

This review focuses on the interaction of positrons with molecules in the range of energies below the thresholds for electronic excitation and Ps formation. Experimental studies of annihilation resolved as a function of positron energy show that positrons bind to many molecular species. This enables the formation of longlived resonant states (i.e., vibrational Feshbach resonances) in two-body positron-molecule collisions, thus altering the dynamics in a fundamental way for the incident positron energies in the range of the molecular vibrations.

These VFR are responsible for the large annihilation rates observed in many polyatomic molecules. They exceed by orders of magnitude the contribution of direct, "in-flight" annihilation. This enhancement distinguishes 
annihilation from conventional scattering processes. For example, the resonant contribution would be hard (if at all possible) to observe in elastic scattering, where much larger contributions come from potential scattering.

Theoretically, resonant annihilation can be described using a Breit-Wigner approach. The key quantities required to make predictions are energies of the VFR and their decay rates (i.e., the annihilation rate, positron capture and autodetachment rates, etc.). Such a calculation can be done for small molecules (e.g., the methyl halides and similar molecules with IR-active modes) using a theory which makes use of the small parameter in the problem, namely, the positron binding energy. This enables the use of long-range dipole coupling to evaluate the positron elastic capture rate $\Gamma^{e}$ and the (universal) $\sqrt{\varepsilon_{b}}$ scaling to estimate the positron annihilation rate $\Gamma^{a}$. The theory also makes use of the fact that the vibrational spectra are sufficiently simple and that the IR strengths of the modes are known. The only free parameter is the positron-molecule binding energy, which can be determined experimentally. In the case of methyl halides, the theoretical predictions are in excellent agreement with the experimentally measured annihilation rates as a function of positron energy. Application to the deuterated methyl halides provides a complete test of the theory with no adjustable parameters. The theory has also been successfully extended to include combination and overtone vibrations.

A general result of the theory is that the resonances for all modes with coupling strengths $\Gamma^{e} \gg \Gamma^{a}$ produce annihilation resonances of the same magnitude, modulated only by the factor $g=\sqrt{\varepsilon_{b} / \varepsilon}$. There are no known cases where dipole coupling strengths greater than $\Gamma^{a}$ fail to produce resonances. On the other hand, the theory is incomplete for molecules such as ethylene and acetylene, where explaining the measured annihilation spectra requires the inclusion of VFR associated with nominally IR-inactive modes and overtones and combination vibrations. In these cases, the couplings are more difficult to evaluate.

As the number of atoms $N$ in the molecule is increased, the magnitudes of the annihilation resonances exceed (often greatly) those explicable on the basis of individual single-mode resonances. The annihilation rates in these species scale as $Z_{\text {eff }} \propto g N^{4.1}$. While a quantitative explanation for this $N^{4}$ scaling is lacking, it is likely that the process of intramolecular vibrational energy redistribution is responsible for this enhancement. This IVR process couples the single-mode resonant doorway states to baths of dark states, namely, states that are not directly coupled to the positron continuum. In this picture, $N$ likely reflects the number of vibrational degrees of freedom of the molecule.

For attachment and annihilation in large molecules, there is a related key piece of the theory that is missing. If the positrons were able to populate the VFRs associated with all possible vibrational excitations, $Z_{\text {eff }}$ would be expected to increase much faster with molecular size than is observed. While vibrationally inelastic escape channels could moderate such growth, they appear to be generally inoperative. Hence, it is not known presently what subset of multimode dark states and subsequent positron escape channels are accessible. By inference from the experimental results, detachment from these dark states likely takes place via a quasielastic escape channel or channels, including the original and nearby doorway states (e.g., dipole-allowed modes close in energy to the doorway state through which the positron entered).

The energy-resolved annihilation data have provided measurements of positron-molecule binding energies $\varepsilon_{b}$ for 30 molecular species. The molecular dipole polarizability plays an important role in fixing the magnitude of $\varepsilon_{b}$. Binding is further enhanced in aromatic molecules by an amount that increases with the number of electronic $\pi$ bonds. In small molecules, the permanent dipole moment also increases $\varepsilon_{b}$. These results indicate that most large molecules will bind positrons, and they provide some insight into promising candidates for further experimental and theoretical study. The goal of finding molecular species for which the binding energy can be both calculated and measured is closer to realization with the recent discovery of small molecules with relatively large binding energies (Danielson et al., 2010). Other interesting topics for further research include study of very large molecules, such as polycyclic aromatic hydrocarbons that are of astrophysical interest (Iwata et al., 1996; Guessoum et al., 2010), and cagelike structures, such as $\mathrm{C}_{60}$ (Gianturco and Lucchese, 1999).

Following positron annihilation, VFR-mediated or not, the remaining molecular ion is frequently found to fragment. There are many open questions in this area, including what determines the degree of fragmentation. A practical question of interest is whether positroninduced annihilation might be a way to produce unfragmented ions from large molecular species for applications such as mass spectrometry.

Finally, the resonant enhancement of positron annihilation described here has an electron analog. In electron-molecule scattering, resonances are known to drive processes such as dissociative attachment (Christophorou et al., 1984), a process that involves the slow motion of the heavy atomic nuclei and that would be weak in a direct electron scattering process. In many electrondriven reactions, shape resonances are prominent. While this is not the case for positrons, both positrons and electrons can populate VFR directly. In the positron case, even a weak positron-vibrational coupling is sufficient to "turn on" the resonant annihilation mechanism. It is possible that a better understanding of positron attachment to polyatomic molecules, gained through such annihilation studies, can be useful in understanding similar electron-molecule processes.

\section{ACKNOWLEDGMENTS}

We are indebted to many colleagues for their contributions to the topics reviewed here. In particular, we acknowledge the collaboration of L. D. Barnes, J. R. 
Danielson, L. Dunlop, P. Gill, R. G. Greaves, D. Green, K. Iwata, C. Kurz, C. M. R. Lee, M. Leventhal, J. Ludlow, J. Marler, T. J. Murphy, A. Passner, and J. P. Sullivan. We also gladly acknowledge helpful conversations with M. Allan, M. Bromley, S. Buckman, M. Charlton, P. Coleman, I. Fabrikant, F. Gianturco, H. Hotop, and M. Lima. G.F.G. is grateful to V. Flambaum for arousing his interest in the problem of low-energy positron interaction with atoms and molecules and for much of what he learned during seven years at UNSW (Sydney, Australia). The experimental work at UCSD was supported by the NSF, Grant No. PHY 07-55809. The work at UCSD benefited greatly from the technical assistance of E. A. Jerzewski.

\section{REFERENCES}

Abdoul-Carime, H., and C. Desfrançois, 1998, Eur. Phys. J. D 2, 149.

Abdoul-Carime, H., J. P. Schermann, and C. Desfrançois, 2002, Few-Body Syst. 31, 183.

Adamson, P. E., X. F. Duan, L. W. Burggraf, M. V. Pak, C. Swalina, and S. Hammes-Schiffer, 2008, J. Phys. Chem. A 112, 1346.

Allan, M., 1984, Chem. Phys. 84, 311.

Al-Qaradawi, I., M. Charlton, and I. Borozan, 2000, J. Phys. B 33, 2725.

Armour, E. A. G., 1984, J. Phys. B 17, L375.

Armour, E. A. G., and D. J. Baker, 1985, J. Phys. B 18, L845.

Armour, E. A. G., and D. J. Baker, 1986, J. Phys. B 19, L871.

Armour, E. A. G., D. J. Baker, and M. Plummer, 1990, J. Phys. B 23, 3057.

Arponen, J., 1978, J. Phys. C 11, L739.

Bardsley, J. N., 1968a, J. Phys. B 1, 349.

Bardsley, J. N., 1968b, J. Phys. B 1, 365.

Barnes, L. D., 2004, Ph.D. thesis (University of California).

Barnes, L. D., S. J. Gilbert, and C. M. Surko, 2003, Phys. Rev. A 67, 032706.

Barnes, L. D., J. A. Young, and C. M. Surko, 2006, Phys. Rev. A 74, 012706.

Berestetskii, V. B., E. M. Lifshitz, and L. P. Pitaevskii, 1982, Quantum Electrodynamics, 2nd ed. (Pergamon, Oxford).

Bertie, J. E., and S. L. Zhang, 1997, J. Mol. Struct. 333, 413.

Bishop, L. M., and L. M. Cheung, 1982, J. Phys. Chem. Ref. Data 11, 119.

Bohr, A., and B. Mottelson, 1998, Nuclear Structure (World Scientific, Singapore), Vol. 1, p. 434.

Boroński, E., and R. M. Nieminen, 1986, Phys. Rev. B 34, 3820.

Bressanini, D., M. Mella, and G. Morosi, 1998, J. Chem. Phys. 109, 1716.

Bromley, M. W. J., and J. Mitroy, 2000, J. Phys. B 33, L325.

Bromley, M. W. J., and J. Mitroy, 2002, Phys. Rev. A 66, 062504.

Bromley, M. W. J., and J. Mitroy, 2006, Phys. Rev. A 73, 032507.

Bromley, M. W. J., and J. Mitroy, 2007, Phys. Rev. A 75, 042506.

Bromley, M. W. J., and J. Mitroy, 2010, Phys. Rev. A 81, 052708.

Brown, B. L., W. S. Crane, and A. P. Mills, 1986, Appl. Phys. Lett. 48, 739.

Bubin, S., and L. Adamowicz, 2004, J. Chem. Phys. 120, 6051.
Buenker, R. J., and H.-P. Liebermann, 2008, Nucl. Instrum. Methods Phys. Res. B 266, 483.

Buenker, R. J., H.-P. Liebermann, V. Melnikov, M. Tachikawa, L. Pichl, and M. Kimura, 2005, J. Phys. Chem. A 109, 5956. Buenker, R. J., H.-P. Liebermann, L. Pichl, M. Tachikawa, and M. Kimura, 2007, J. Chem. Phys. 126, 104305.

Buenker, R. J., H.-P. Liebermann, M. Tachikawa, L. Pichl, and M. Kimura, 2006, Nucl. Instrum. Methods Phys. Res. B 247, 47.

Carey, R., R. R. Lucchese, and F. A. Gianturco, 2008, Phys. Rev. A 78, 012706.

Cassidy, D. B., and A. P. Mills, 2007, Nature (London) 449, 195. Charlton, M., T. C. Griffith, G. R. Heyland, K. S. Lines, and G. L. Wright, 1980, J. Phys. B 13, L757.

Charlton, M., and J. W. Humberston, 2001, Positron Physics (Cambridge University Press, Cambridge).

Charlton, M., D. P. van der Werf, and I. Al-Qaradawi, 2002, Phys. Rev. A 65, 042716.

Charlton, M., D. P. van der Werf, R. J. Lewis, P. R. Watkeys, and S. J. Kerrigan, 2006, J. Phys. B 39, L329.

Chojnacki, H., and K. Strasburger, 2006, Mol. Phys. 104, 2273. Christophorou, L. G., D. L. McCorkle, and A. A. Christodoulides, 1984, in Electron-Molecule Interactions and Their Applications, edited by L. G. Christophorou (Academic, New York), Chap. 6, p. 477.

Churazov, E., R. Sunyaev, S. Sazonov, M. Revnivtsev, and D. Varshalovich, 2005, Mon. Not. R. Astron. Soc. 357, 1377.

Coleman, P. G., 2000, Ed., Positron Beams and Their Applications (World Scientific, Singapore).

Coleman, P. G., S. Rayner, F. M. Jacobsen, M. Charlton, and R. N. West, 1994, J. Phys. B 27, 981.

Crawford, O. H., 1967, Proc. Phys. Soc. London 91, 279.

Crawford, O. H., 1994, Phys. Rev. A 49, R3147.

Crowder, G. A., and J. M. Lightfoot, 1983, J. Mol. Struct. 99, 77.

Danby, G., and J. Tennyson, 1988, Phys. Rev. Lett. 61, 2737.

Danielson, J. R., J. J. Gosselin, and C. M. Surko, 2010, Phys. Rev. Lett. 104, 233201.

Danielson, J. R., J. A. Young, and C. M. Surko, 2009, J. Phys. B 42, 235203.

da Silva, E. P., J. S. E. Germano, and M. A. P. Lima, 1994, Phys. Rev. A 49, R1527.

da Silva, E. P., J. S. E. Germano, and M. A. P. Lima, 1996, Phys. Rev. Lett. 77, 1028.

David, A., G. Kögel, P. Sperr, and W. Triftshäuser, 2001, Phys. Rev. Lett. 87, 067402.

de Carvalho, C. R. C., M. T. do N. Varella, M. A. P. Lima, and E. P. da Silva, 2003, Phys. Rev. A 68, 062706.

de Carvalho, C. R. C., M. T. do N. Varella, M. A. P. Lima, E. P. da Silva, and J. S. E. Germano, 2000, Nucl. Instrum. Methods Phys. Res. B 171, 33.

Demkov, Y. N., and V. N. Ostrovsky, 1988, Zero-Range Potentials and Their Applications in Atomic Physics (Plenum, New York).

Desfrançois, C., H. Abdoul-Carime, N. Khelifa, and J. P. Schermann, 1994, Phys. Rev. Lett. 73, 2436.

Desfrançois, C., G. Grégoire, and J.-P. Schermann, 2004, FewBody Syst. 34, 169.

Deutsch, M., 1951a, Phys. Rev. 82, 455.

Deutsch, M., 1951b, Phys. Rev. 83, 866.

Deutsch, M., 1953, Prog. Nucl. Phys. 3, 131.

Dirac, P. A. M., 1930, Proc. Cambridge Philos. Soc. 26, 361.

Dlubek, G., K. Saarinen, and H. M. Fretwell, 1998, J. Polym. 
Sci. B 36, 1513.

Domcke, W., 1981, J. Phys. B 14, 4889.

Donohue, D. L., L. D. Hulett, B. A. Eckenrode, S. A. McLuckey, and G. L. Glish, 1990, Chem. Phys. Lett. 168, 37. Dunlop, L. J. M., and G. F. Gribakin, 2006, J. Phys. B 39, 1647. Dupasquier, A., and A. P. Mills, 1995, Eds., Positron Spectroscopy of Solids (IOS, Amsterdam).

Durig, J. R., G. A. Guirgis, and Y. S. Li, 1981, J. Chem. Phys. 74, 5946 .

Dzuba, V. A., V. V. Flambaum, G. F. Gribakin, and C. Harabati, 1999, Phys. Rev. A 60, 3641.

Dzuba, V. A., V. V. Flambaum, G. F. Gribakin, and W. A. King, 1995, Phys. Rev. A 52, 4541.

Dzuba, V. A., V. V. Flambaum, G. F. Gribakin, and W. A. King, 1996, J. Phys. B 29, 3151.

Dzuba, V. A., V. V. Flambaum, W. A. King, B. N. Miller, and O. P. Sushkov, 1993, Phys. Scr. T46, 248.

Eades, J., and F. J. Hartmann, 1999, Rev. Mod. Phys. 71, 373.

Fabrikant, I. I., 1977, J. Phys. B 10, 1761.

Fabrikant, I. I., 2005, J. Phys. B 38, 1745.

Fabrikant, I. I., and H. Hotop, 2005, Phys. Rev. Lett. 94, 063201.

Ferrell, R. A., 1956, Rev. Mod. Phys. 28, 308.

Franz, J., and F. A. Gianturco, 2006, Nucl. Instrum. Methods Phys. Res. B 247, 20.

Fraser, P. A., 1968, Adv. At. Mol. Phys. 4, 63.

Fraser, P. A., B. H. Bransden, P. G. Coleman, and W. Raith, 1982, Can. J. Phys. 60, 565.

Garrett, W. R., 1971, Phys. Rev. A 3, 961.

Georges, R., M. Bach, and M. Herman, 1999, Mol. Phys. 97, 279.

Germano, J. S. E., and M. A. P. Lima, 1993, Phys. Rev. A 47, 3976.

Gianturco, F. A., J. Franz, R. J. Buenker, H.-P. Liebermann, L. Pichl, J.-M. Rost, M. Tachikawa, and M. Kimura, 2006, Phys. Rev. A 73, 022705.

Gianturco, F. A., and R. R. Lucchese, 1999, Phys. Rev. A 60, 4567.

Gianturco, F. A., and T. Mukherjee, 1999, Europhys. Lett. 48, 519.

Gianturco, F. A., and T. Mukherjee, 2000, Nucl. Instrum. Methods Phys. Res. B 171, 17.

Gianturco, F. A., T. Mukherjee, and A. Occhigrossi, 2001, Phys. Rev. A 64, 032715.

Gianturco, F. A., P. Nichols, T. L. Gibson, and R. R. Lucchese, 2005, Phys. Rev. A 72, 032724.

Gidley, D. W., T. L. Dull, W. E. Frieze, J. Sun, A. F. Yee, C. V. Nguyen, and D. Y. Yoon, 2000, Appl. Phys. Lett. 76, 1282.

Gidley, D. W., H. G. Peng, and R. S. Vallery, 2006, Annu. Rev. Mater. Res. 36, 49.

Gilbert, S. J., 2000, Ph.D. thesis (University of California).

Gilbert, S. J., L. D. Barnes, J. P. Sullivan, and C. M. Surko, 2002, Phys. Rev. Lett. 88, 043201.

Gilbert, S. J., C. Kurz, R. G. Greaves, and C. M. Surko, 1997, Appl. Phys. Lett. 70, 1944.

Glish, G. L., R. G. Greaves, S. A. McLuckey, L. D. Hulett, C. M. Surko, J. Xu, and D. L. Donohue, 1994, Phys. Rev. A 49, 2389.

Goldanskii, V. I., and Y. S. Sayasov, 1964, Phys. Lett. 13, 300. Greaves, R. G., and C. M. Surko, 1996, Can. J. Phys. 51, 445. Greaves, R. G., and C. M. Surko, 2000, Phys. Rev. Lett. 85, 1883.

Gribakin, G. F., 2000, Phys. Rev. A 61, 022720.
Gribakin, G. F., 2001, in New Directions in Antimatter Chemistry and Physics, edited by C. M. Surko and F. A. Gianturco (Kluwer Academic, Dordrecht), Chap. 22, p. 413.

Gribakin, G. F., 2002, Nucl. Instrum. Methods Phys. Res. B 192, 26.

Gribakin, G. F., 2009, unpublished.

Gribakin, G. F., 2010, J. Phys.: Conf. Ser. 199, 012013.

Gribakin, G. F., and P. M. W. Gill, 2004, Nucl. Instrum. Methods Phys. Res. B 221, 30.

Gribakin, G. F., and C. M. R. Lee, 2006a, Phys. Rev. Lett. 97, 193201.

Gribakin, G. F., and C. M. R. Lee, 2006b, Nucl. Instrum. Methods Phys. Res. B 247, 31.

Gribakin, G. F., and C. M. R. Lee, 2009, Eur. Phys. J. D 51, 51. Gribakin, G. F., and J. Ludlow, 2004, Phys. Rev. A 70, 032720. Griffith, T. C., and G. R. Heyland, 1978, Phys. Rep. 39, 169.

Guessoum, N., P. Jean, and W. Gillard, 2010, Mon. Not. R. Astron. Soc. 402, 1171.

Guirgis, G. A., X. Zhu, and J. R. Durig, 1999, Struct. Chem. 10, 445.

Gullikson, E. M., and A. P. Mills, 1987, Phys. Rev. B 36, 8777. Gutowski, M., K. D. Jordan, and P. Skurski, 1998, J. Phys. Chem. A 102, 2624.

Gutsev, G. L., M. Nooijen, and R. J. Bartlett, 1997, Chem. Phys. Lett. 276, 13.

Heyland, G. R., M. Charlton, S. A. Davies, and T. C. Griffith, 1986, Phys. Lett. A 119, 289.

Heyland, G. R., M. Charlton, T. C. Griffith, and G. Clark, 1985, Chem. Phys. 95, 157.

Heyland, G. R., M. Charlton, T. C. Griffith, and G. L. Wright, 1982, Can. J. Phys. 60, 503.

Hotop, H., M. W. Ruf, M. Allan, and I. I. Fabrikant, 2003, Adv. At. Mol. Phys. 49, 85.

Hulett, L. D., D. L. Donohue, J. Xu, T. A. Lewis, S. A. McLuckey, and G. L. Glish, 1993, Chem. Phys. Lett. 216, 236. Hulett, L. D., J. Xu, S. A. McLuckey, T. A. Lewis, and D. M. Schrader, 1996, Can. J. Phys. 74, 411.

Hulett, L. D., J. Xu, S. A. McLuckey, and D. M. Schrader, 1996, J. Radioanal. Nucl. Chem. 210, 309.

Iakubov, I. T., and A. G. Khrapak, 1982, Rep. Prog. Phys. 45, 697.

Iwata, K., 1997, Ph.D. thesis (University of California).

Iwata, K., R. G. Greaves, T. J. Murphy, M. D. Tinkle, and C. M. Surko, 1995, Phys. Rev. A 51, 473.

Iwata, K., R. G. Greaves, and C. M. Surko, 1994, Hyperfine Interact. 89, 271.

Iwata, K., R. G. Greaves, and C. M. Surko, 1996, Can. J. Phys. 74, 407.

Iwata, K., R. G. Greaves, and C. M. Surko, 1997, Phys. Rev. A 55, 3586 .

Iwata, K., G. F. Gribakin, R. G. Greaves, C. Kurz, and C. M. Surko, 2000, Phys. Rev. A 61, 022719.

Iwata, K., G. F. Gribakin, R. G. Greaves, and C. M. Surko, 1997, Phys. Rev. Lett. 79, 39.

Jain, A., and F. A. Gianturco, 1991, J. Phys. B 24, 2387.

Jain, A., and D. G. Thompson, 1983, J. Phys. B 16, 1113.

Kim, H. L., T. J. Kulp, and J. D. McDonald, 1987, J. Chem. Phys. 87, 4376.

Kita, Y., R. Maezono, M. Tachikawa, M. Towler, and R. J. Needs, 2009, J. Chem. Phys. 131, 134310.

Klar, D., M.-W. Ruf, and H. Hotop, 1992a, Aust. J. Phys. 45, 263.

Klar, D., M.-W. Ruf, and H. Hotop, 1992b, Chem. Phys. Lett. 
189, 448

Klar, D., M.-W. Ruf, and H. Hotop, 1994, Meas. Sci. Technol. $\mathbf{5}, 1248$.

Kong, J., et al., 2000, J. Comput. Chem. 21, 1532.

Kurtz, H. A., and K. D. Jordan, 1978, J. Phys. B 11, L479.

Kurtz, H. A., and K. D. Jordan, 1981, J. Chem. Phys. 75, 1876.

Kurz, C., S. J. Gilbert, R. G. Greaves, and C. Surko, 1998, Nucl. Instrum. Methods Phys. Res. B 143, 188.

Kurz, C., R. G. Greaves, and C. M. Surko, 1996, Phys. Rev. Lett. 77, 2929.

Landau, L. D., and E. M. Lifshitz, 1977, Quantum Mechanics, 3rd ed. (Pergamon, Oxford).

Lane, N. F., 1980, Rev. Mod. Phys. 52, 29.

Laricchia, G., and C. Wilkin, 1997, Phys. Rev. Lett. 79, 2241.

Leber, E., S. Barsotti, I. I. Fabrikant, J. M. Weber, M.-W. Ruf, and H. Hotop, 2000, Eur. Phys. J. D 12, 125.

Lee, C., 1957, Zh. Eksp. Teor. Fiz. 33, 365 [Sov. Phys. JETP 6, 281 (1958)].

Lide, D. R., 2000, Ed., Handbook of Chemistry and Physics, 81st ed. (CRC, Boca Raton, FL).

Lino, J. L. S., J. S. E. Germano, E. P. da Silva, and M. A. P. Lima, 1998, Phys. Rev. A 58, 3502.

Linstrom, P. J., and W. G. Mallard, 2005, Eds., NIST Chemistry WebBook, NIST Standard Reference Database Number 69 (NIST, Gaithesburg, MD).

Major, Z., S. B. Dugdale, R. J. Watts, G. Santi, M. A. Alam, S. M. Hayden, J. A. Duffy, J. W. Taylor, T. Jarlborg, E. Bruno, D. Benea, and H. Ebert, 2004, Phys. Rev. Lett. 92, 107003.

Marler, J. P., 2005, Ph.D. thesis (University of California).

Marler, J. P., G. F. Gribakin, and C. Surko, 2006, Nucl. Instrum. Methods Phys. Res. B 247, 87.

Marler, J. P., and C. M. Surko, 2005, Phys. Rev. A 72, 062702.

Massey, H. S. W., 1982, Can. J. Phys. 60, 461.

McClellan, A. L., 1963, Tables of Experimental Dipole Moments (Freeman, San Francisco).

McIlroy, A., and D. J. Nesbitt, 1990, J. Chem. Phys. 92, 2229.

McNutt, J. D., S. C. Sharma, and R. D. Brisbon, 1979, Phys. Rev. A 20, 347.

McNutt, J. D., V. B. Summerour, A. D. Ray, and P. H. Huang, 1975, J. Chem. Phys. 62, 1777.

Mella, M., D. Bressanini, and G. Morosi, 2001, J. Chem. Phys. 114, 10579.

Mella, M., G. Morosi, D. Bressanini, and S. Elli, 2000, J. Chem. Phys. 113, 6154.

Miller, K. J., 1990, J. Am. Chem. Soc. 112, 8533.

Mills, A. P., 2002, Nucl. Instrum. Methods Phys. Res. B 192, 107.

Mills, A. P., 2007, Radiat. Phys. Chem. 76, 76.

Mills, A. P., D. B. Cassidy, and R. G. Greaves, 2004, Mater. Sci. Forum 445-446, 424.

Mitroy, J., 2002, Phys. Rev. A 66, 022716.

Mitroy, J., and M. W. J. Bromley, 2007, Phys. Rev. Lett. 98 173001.

Mitroy, J., M. W. J. Bromley, and G. Ryzhikh, 1999, J. Phys. B 32, 2203.

Mitroy, J., M. W. J. Bromley, and G. G. Ryzhikh, 2002, J. Phys. B 35, R81.

Mitroy, J., and I. A. Ivanov, 2002, Phys. Rev. A 65, 042705.

Mitroy, J., and G. Ryzhikh, 1999, J. Phys. B 32, 2831.

Mitroy, J., and G. G. Ryzhikh, 2000, J. Phys. B 33, 3495.

Mitroy, J., J. Y. Zhang, M. W. J. Bromley, and S. I. Young, 2008, Phys. Rev. A 78, 012715.

Moxom, J., D. M. Schrader, G. Laricchia, J. Xu, and L. D.
Hulett, 2000, Phys. Rev. A 62, 052708.

Murphy, T. J., and C. M. Surko, 1990, J. Phys. B 23, L727.

Murphy, T. J., and C. M. Surko, 1991, Phys. Rev. Lett. 67, 2954.

Murphy, T. J., and C. M. Surko, 1992, Phys. Rev. A 46, 5696.

Nesbitt, D. J., and R. W. Field, 1996, J. Phys. Chem. 100, 12735.

Nishimura, T., and F. A. Gianturco, 2003, Phys. Rev. Lett. 90, 183201.

Nishimura, T., and F. A. Gianturco, 2004, Eur. Phys. J. D 68, 377.

Nishimura, T., and F. A. Gianturco, 2005a, Eur. Phys. J. D 33, 221.

Nishimura, T., and F. A. Gianturco, 2005b, Phys. Rev. A 72, 022706.

Occhigrossi, A., and F. A. Gianturco, 2003, J. Phys. B 36, 1383.

O'Malley, T. F., 1966, Phys. Rev. 150, 14.

Osmon, P. E., 1965a, Phys. Rev. 140, A8.

Osmon, P. E., 1965b, Phys. Rev. 138, B216.

Passner, A., C. M. Surko, M. Leventhal, and A. P. Mills, 1989, Phys. Rev. A 39, 3706.

Paul, D. A. L., and L. Saint-Pierre, 1963, Phys. Rev. Lett. 11, 493.

Pichl, L., M. Tachikawa, R. J. Buenker, M. Kimura, and J.-M. Rost, 2005, IEEE Trans. Nucl. Sci. 52, 2810.

Pomeranchuk, I., 1949, Zh. Eksp. Teor. Fiz. 19, 183.

Puska, M. J., and R. M. Nieminen, 1994, Rev. Mod. Phys. 66, 841.

Ramaty, R., M. Leventhal, K. W. Chan, and R. E. Lingenfelter, 1992, Astrophys. J. Lett. 392, L63.

Rienstra-Kiracofe, J. C., G. S. Tschumper, H. F. Schaefer, S. Nandi, and G. B. Ellison, 2002, Chem. Rev. 102, 231.

Ryzhikh, G., and J. Mitroy, 1998, J. Phys. B 31, 3465.

Ryzhikh, G. G., and J. Mitroy, 1997, Phys. Rev. Lett. 79, 4124. Ryzhikh, G. G., J. Mitroy, and K. Varga, 1998, J. Phys. B 31, 3965.

Sanchez, S. d'A., M. A. P. Lima, and M. T. do N. Varella, 2009, Phys. Rev. A 80, 052710.

Schrader, D. M., and J. Moxom, 2001, in New Directions in Antimatter Chemistry and Physics, edited by C. M. Surko and F. A. Gianturco (Kluwer Academic, Dordrecht), Chap. 15, p. 263.

Schrader, D. M., and C. M. Wang, 1976, J. Phys. Chem. 80, 2507.

Schultz, P. J., and K. G. Lynn, 1988, Rev. Mod. Phys. 60, 701. Sharma, S. C., S. D. Hyatt, M. H. Ward, and C. A. Dark, 1985, J. Phys. B 18, 3245.

Sharma, S. C., and J. D. McNutt, 1978, Phys. Rev. A 18, 1426. Shaw, R. A., H. Wieser, R. Dutler, and A. Rauk, 1990, J. Am. Chem. Soc. 112, 5401.

Shearer, J. W., and M. Deutsch, 1949, Phys. Rev. 76, 462.

Smith, P. M., and D. A. L. Paul, 1970, Can. J. Phys. 48, 2984.

Stewart, G. M., and J. D. McDonald, 1983, J. Chem. Phys. 78, 3907.

Strasburger, K., 1996, Chem. Phys. Lett. 253, 49.

Strasburger, K., 1999, J. Chem. Phys. 111, 10555.

Strasburger, K., 2001, J. Chem. Phys. 114, 615.

Strasburger, K., 2004, Struct. Chem. 15, 415.

Stuchebrukhov, A. A., and R. A. Marcus, 1993, J. Chem. Phys. 98, 6044.

Sullivan, J. P., L. D. Barnes, J. P. Marler, S. J. Gilbert, and C. M. Surko, 2004, Mater. Sci. Forum 445-446, 435.

Sullivan, J. P., S. J. Gilbert, J. P. Marler, L. D. Barnes, S. J. Buckman, and C. M. Surko, 2002, Nucl. Instrum. Methods Phys. Res. B 192, 3. 
Sullivan, J. P., S. J. Gilbert, J. P. Marler, R. G. Greaves, S. J. Buckman, and C. M. Surko, 2002, Phys. Rev. A 66, 042708.

Surko, C. M., S. J. Gilbert, and R. G. Greaves, 1999, in NonNeutral Plasma Physics III, edited by J. J. Bollinger, R. L. Spencer, and R. C. Davidson, AIP Conf. Proc. No. 498 (AIP, Melville, NY), pp. 3-12.

Surko, C. M., and R. G. Greaves, 2004, Phys. Plasmas 11, 2333.

Surko, C. M., G. F. Gribakin, and S. J. Buckman, 2005, J. Phys. B 38, R57.

Surko, C. M., M. Leventhal, A. Passner, and F. J. Wysocki, 1988, in Non-Neutral Plasma Physics, edited by C. W. Roberson and C. F. Driscoll, AIP Conf. Proc. No. 175 (AIP, New York), p. 75.

Surko, C. M., A. Passner, M. Leventhal, and F. J. Wysocki, 1988, Phys. Rev. Lett. 61, 1831.

Tachikawa, M., R. J. Buenker, and M. Kimura, 2003, J. Chem. Phys. 119, 5005.

Tachikawa, M., Y. Kita, and R. J. Buenker, 2010, private communication.

Tachikawa, M., I. Shimamura, R. J. Buenker, and M. Kimura, 2001, in New Directions in Antimatter Chemistry and Physics, edited by C. M. Surko and F. A. Gianturco (Kluwer Academic, Dordrecht), Chap. 23, p. 437.

Tang, S., M. D. Tinkle, R. G. Greaves, and C. M. Surko, 1992, Phys. Rev. Lett. 68, 3793.

Tao, S. J., 1965, Phys. Rev. Lett. 14, 935.

Tao, S. J., 1970, Phys. Rev. A 2, 1669.

Uzer, T., and W. H. Miller, 1991, Phys. Rep. 199, 73.

Varella, M. T. do N., C. R. C. de Carvalho, and M. A. P. Lima, 2002, Nucl. Instrum. Methods Phys. Res. B 192, 225.

Wahl, R. L., 2002, Principles and Practice of Positron Emission Tomography (Lippincott, Williams and Wilkins, Philadelphia).
Wang, F., 2003, J. Phys. Chem. A 107, 10199.

Wang, F., S. Saha, D. G. Green, G. F. Gribakin, and C. M. Surko, 2010, unpublished.

Weber, J. M., E. Leber, M.-W. Ruf, and H. Hotop, 1999, Phys. Rev. Lett. 82, 516.

Wright, G. L., M. Charlton, G. Clark, T. C. Griffith, and G. R. Heyland, 1983, J. Phys. B 16, 4065.

Wright, G. L., M. Charlton, T. C. Griffith, and G. R. Heyland, 1985, J. Phys. B 18, 4327.

$\mathrm{Xu}$, J., L. D. Hulett, T. A. Lewis, D. L. Donohue, S. A. McLuckey, and O. H. Crawford, 1994, Phys. Rev. A 49, R3151.

Xu, J., L. D. Hulett, T. A. Lewis, D. L. Donohue, S. A. McLuckey, and G. L. Glish, 1993, Phys. Rev. A 47, 1023.

Xu, J., L. D. Hulett, T. A. Lewis, and S. A. McLuckey, 1995, Phys. Rev. A 52, 2088.

Xu, J., L. D. Hulett, J. Moxom, W. Wu, S. Datz, and D. M. Schrader, 1997, Phys. Rev. A 56, R4373.

Yamada, Y., Y. Katsumoto, and T. Ebata, 2007, Phys. Chem. Chem. Phys. 9, 1170.

Young, J. A., 2007, Ph.D. thesis (University of California).

Young, J. A., G. F. Gribakin, C. M. R. Lee, and C. M. Surko, 2008, Phys. Rev. A 77, 060702R.

Young, J. A., and C. M. Surko, 2007, Phys. Rev. Lett. 99, 133201.

Young, J. A., and C. M. Surko, 2008a, Nucl. Instrum. Methods Phys. Res. B 266, 478.

Young, J. A., and C. M. Surko, 2008b, Phys. Rev. A 77, 052704. Young, J. A., and C. M. Surko, 2008c, Phys. Rev. A 78, 032702. Zhang, J.-Y., J. Mitroy, and K. Varga, 2009, Phys. Rev. Lett. 103, 223202. 